\title{
ON THE PROBABILISTIC CAUCHY THEORY OF THE CUBIC NONLINEAR SCHRÖDINGER EQUATION ON $\mathbb{R}^{d}, d \geq 3$
}

\author{
ÁRPÁD BÉNYI, TADAHIRO OH, AND OANA POCOVNICU
}

\begin{abstract}
We consider the Cauchy problem of the cubic nonlinear Schrödinger equation (NLS) : $i \partial_{t} u+\Delta u= \pm|u|^{2} u$ on $\mathbb{R}^{d}, d \geq 3$, with random initial data and prove almost sure well-posedness results below the scaling-critical regularity $s_{\text {crit }}=\frac{d-2}{2}$. More precisely, given a function on $\mathbb{R}^{d}$, we introduce a randomization adapted to the Wiener decomposition, and, intrinsically, to the so-called modulation spaces. Our goal in this paper is three-fold. (i) We prove almost sure local well-posedness of the cubic NLS below the scalingcritical regularity along with small data global existence and scattering. (ii) We implement a probabilistic perturbation argument and prove 'conditional' almost sure global well-posedness for $d=4$ in the defocusing case, assuming an a priori energy bound on the critical Sobolev norm of the nonlinear part of a solution; when $d \neq 4$, we show that conditional almost sure global wellposedness in the defocusing case also holds under an additional assumption of global well-posedness of solutions to the defocusing cubic NLS with deterministic initial data in the critical Sobolev regularity. (iii) Lastly, we prove global well-posedness and scattering with a large probability for initial data randomized on dilated cubes.
\end{abstract}

\section{Contents}

1. Introduction

2. Probabilistic lemmata

3. Function spaces and their properties

4. Probabilistic nonlinear estimates

5. Proofs of Theorems 1.1 and 1.2

6. Local well-posedness of NLS with a deterministic perturbation

7. Long time existence of solutions to the perturbed NLS

8. Proof of Theorem 1.3

9. Probabilistic global existence via randomization on dilated cubes

Appendix A. On the properties of the $U^{p}$ - and $X^{s}$-spaces

References

Received by the editors October 27, 2014 and, in revised form, April 20, 2015.

2010 Mathematics Subject Classification. Primary 35Q55.

Key words and phrases. Nonlinear Schrödinger equation, almost sure well-posedness, modulation space, Wiener decomposition.

This work was partially supported by a grant from the Simons Foundation (No. 246024 to the first author). The second author was supported by the European Research Council (grant no. 637995 "ProbDynDispEq"). The third author was supported by the NSF grant under agreement No. DMS-1128155. Any opinions, findings, and conclusions or recommendations expressed in this material are those of the authors and do not necessarily reflect the views of the NSF.

(C) 2015 by the authors under Creative Commons Attribution 3.0 License (CC BY 3.0) 


\section{INTRODUCTION}

1.1. Background. In this paper, we consider the Cauchy problem of the cubic nonlinear Schrödinger equation (NLS) on $\mathbb{R}^{d}, d \geq 3$ :

$$
\left\{\begin{array}{l}
i \partial_{t} u+\Delta u= \pm \mathcal{N}(u), \\
\left.u\right|_{t=0}=u_{0} \in H^{s}\left(\mathbb{R}^{d}\right),
\end{array} \quad(t, x) \in \mathbb{R} \times \mathbb{R}^{d},\right.
$$

where $\mathcal{N}(u):=|u|^{2} u$. The cubic NLS (1.1) has been studied extensively from both the theoretical and applied points of view. Our main focus is to study well-posedness of (1.1) with random and rough initial data.

It is well known that the cubic NLS (1.1) enjoys the dilation symmetry. More precisely, if $u(t, x)$ is a solution to (1.1) on $\mathbb{R}^{d}$ with an initial condition $u_{0}$, then

$$
u_{\mu}(t, x):=\mu^{-1} u\left(\mu^{-2} t, \mu^{-1} x\right)
$$

is also a solution to (1.1) with the $\mu$-scaled initial condition $u_{0, \mu}(x):=\mu^{-1} u_{0}\left(\mu^{-1} x\right)$. Associated to this dilation symmetry, there is the so-called scaling-critical Sobolev index $s_{\text {crit }}:=\frac{d-2}{2}$ such that the homogeneous $\dot{H}^{s_{\text {crit }}}$-norm is invariant under this dilation symmetry. In general, we have

$$
\left\|u_{0, \mu}\right\|_{\dot{H}^{s}\left(\mathbb{R}^{d}\right)}=\mu^{\frac{d-2}{2}-s}\left\|u_{0}\right\|_{\dot{H}^{s}\left(\mathbb{R}^{d}\right)} .
$$

If an initial condition $u_{0}$ is in $H^{s}\left(\mathbb{R}^{d}\right)$, we say that the Cauchy problem (1.1) is subcritical, critical, or supercritical, depending on whether $s>s_{\text {crit }}, s=s_{\text {crit }}$, or $s<s_{\text {crit }}$, respectively.

Let us first discuss the (sub)critical regime. In this case, (1.1) is known to be locally well-posed. See Cazenave-Weissler [17] for local well-posedness of (1.1) in the critical Sobolev spaces. As is well known, the conservation laws play an important role in discussing long time behavior of solutions. There are three known conservation laws for the cubic NLS (1.1):

$$
\begin{aligned}
& \text { Mass: } M[u](t):=\int_{\mathbb{R}^{d}}|u(t, x)|^{2} d x, \\
& \text { Momentum: } P[u](t):=\operatorname{Im} \int_{\mathbb{R}^{d}} u(t, x) \overline{\nabla u(t, x)} d x, \\
& \text { Hamiltonian: } H[u](t):=\frac{1}{2} \int_{\mathbb{R}^{d}}|\nabla u(t, x)|^{2} d x \pm \frac{1}{4} \int_{\mathbb{R}^{d}}|u(t, x)|^{4} d x .
\end{aligned}
$$

The Hamiltonian is also referred to as the energy. In view of the conservation of the energy, the cubic NLS is called energy-subcritical when $d \leq 3\left(s_{\text {crit }}<1\right)$, energycritical when $d=4\left(s_{\text {crit }}=1\right)$, and energy-supercritical when $d \geq 5\left(s_{\text {crit }}>1\right)$, respectively.

In the following, let us discuss the known results on the global-in-time behavior of solutions to the defocusing NLS, corresponding to the + sign in (1.1), in high dimensions $d \geq 3$. When $d=4$, the Hamiltonian is invariant under the scaling (1.2) and plays a crucial role in the global well-posedness theory. Indeed, Ryckman-Vişan [53. proved global well-posedness and scattering for the defocusing cubic NLS on $\mathbb{R}^{4}$. See also Vişan [60]. When $d \neq 4$, there is no known scaling invariant positive conservation law for (1.1) in high dimensions $d \geq 3$. This makes it difficult to study the global-in-time behavior of solutions, in particular, in the scaling-critical regularity. There are, however, 'conditional' global well-posedness and scattering results as we describe below. When $d=3\left(s_{\text {crit }}=\frac{1}{2}\right)$, Kenig-Merle 35] applied 
the concentration compactness and rigidity method developed in their previous paper [34] and proved that if $u \in L_{t}^{\infty} \dot{H}_{x}^{\frac{1}{2}}\left(I \times \mathbb{R}^{3}\right)$, where $I$ is a maximal interval of existence, then $u$ exists globally in time and scatters. For $d \geq 5$, the cubic NLS is supercritical with respect to any known conservation law. Nonetheless, motivated by a similar result of Kenig-Merle [36 on radial solutions to the energysupercritical nonlinear wave equation $(\mathrm{NLW})$ on $\mathbb{R}^{3}$, Killip-Vişan [39] proved that if $u \in L_{t}^{\infty} \dot{H}_{x}^{s_{\text {crit }}}\left(I \times \mathbb{R}^{d}\right)$, where $I$ is a maximal interval of existence, then $u$ exists globally in time and scatters. Note that the results in [35] and 39] are conditional in the sense that they assume an a priori control on the critical Sobolev norm. The question of global well-posedness and scattering without any a priori assumption remains a challenging open problem for $d=3$ and $d \geq 5$.

So far, we have discussed well-posedness in the (sub)critical regularity. In particular, the cubic NLS (1.1) is locally well-posed in the (sub)critical regularity, i.e. $s \geq s_{\text {crit }}$. In the supercritical regime, i.e. $s<s_{\text {crit }}$, on the contrary, (1.1) is known to be ill-posed. See 1, 11, 16, 18. In the following, however, we consider the Cauchy problem (1.1) with initial data in $H^{s}\left(\mathbb{R}^{d}\right), s<s_{\text {crit }}$ in a probabilistic manner. More precisely, given a function $\phi \in H^{s}\left(\mathbb{R}^{d}\right)$ with $s<s_{\text {crit }}$, we introduce a randomization $\phi^{\omega}$ and prove almost sure well-posedness of (1.1).

In studying the Gibbs measure for the defocusing (Wick ordered) cubic NLS on $\mathbb{T}^{2}$, Bourgain [6] considered random initial data of the form:

$$
u_{0}^{\omega}(x)=\sum_{n \in \mathbb{Z}^{2}} \frac{g_{n}(\omega)}{\sqrt{1+|n|^{2}}} e^{i n \cdot x},
$$

where $\left\{g_{n}\right\}_{n \in \mathbb{Z}^{2}}$ is a sequence of independent standard complex-valued Gaussian random variables. The function (1.4) represents a typical element in the support of the Gibbs measure, more precisely, in the support of the Gaussian free field on $\mathbb{T}^{2}$ associated to this Gibbs measure, and is critical with respect to the scaling. With a combination of deterministic PDE techniques and probabilistic arguments, Bourgain showed that the (Wick ordered) cubic NLS on $\mathbb{T}^{2}$ is well-posed almost surely with respect to the random initial data (1.4). In the context of the cubic NLW on a three-dimensional compact Riemannian manifold $M$, Burq-Tzvetkov 14] considered the Cauchy problem with a more general class of random initial data. Given an eigenfunction expansion $u_{0}(x)=\sum_{n=1}^{\infty} c_{n} e_{n}(x) \in H^{s}(M)$ of an initial condition 11 where $\left\{e_{n}\right\}_{n=1}^{\infty}$ is an orthonormal basis of $L^{2}(M)$ consisting of the eigenfunctions of the Laplace-Beltrami operator, they introduced a randomization $u_{0}^{\omega}$ by

$$
u_{0}^{\omega}(x)=\sum_{n=1}^{\infty} g_{n}(\omega) c_{n} e_{n}(x) .
$$

Here, $\left\{g_{n}\right\}_{n=1}^{\infty}$ is a sequence of independent mean-zero random variables with a uniform bound on the fourth moments. Then, they proved almost sure local wellposedness with random initial data of the form (1.5) for $s \geq \frac{1}{4}$. Since the scalingcritical Sobolev index for this problem is $s_{\text {crit }}=\frac{1}{2}$, this result allows us to take initial data below the critical regularity and still construct solutions upon randomization of the initial data. We point out that the randomized function $u_{0}^{\omega}$ in (1.5) has the same Sobolev regularity as the original function $u_{0}$ and is not smoother, almost surely.

\footnotetext{
${ }^{1}$ For NLW, one needs to specify $\left.\left(u, \partial_{t} u\right)\right|_{t=0}$ as an initial condition. For simplicity of presentation, we only discuss $\left.u\right|_{t=0}$.
} 
However, it enjoys a better integrability, which allows one to prove improvements of Strichartz estimates. (See Lemmata 2.2 and 2.3 below.) Such an improvement on integrability for random Fourier series is known as Paley-Zygmund's theorem [49]. See also Kahane 32 and Ayache-Tzvetkov [2]. There are several works on Cauchy problems of evolution equations with random data that followed these results, including some on almost sure global well-posedness: [7, 9, 10, 12, 13, 15, [20, $23,42,45,51,52,58$.

\subsection{Randomization adapted to the Wiener decomposition and modu-} lation spaces. Many of the results mentioned above are on compact domains, where there is a countable basis of eigenfunctions of the Laplacian and thus there is a natural way to introduce a randomization. On $\mathbb{R}^{d}$, there is no countable basis of $L^{2}\left(\mathbb{R}^{d}\right)$ consisting of eigenfunctions of the Laplacian. Randomizations have been introduced with respect to some other countable bases of $L^{2}\left(\mathbb{R}^{d}\right)$, for example, a countable basis of eigenfunctions of the Laplacian with a confining potential such as the harmonic oscillator $\Delta-|x|^{2}$, leading to a careful study of properties of eigenfunctions. In this paper, our goal is to introduce a simple and natural randomization for functions on $\mathbb{R}^{d}$. For this purpose, we first review some basic notions related to the so-called modulation spaces of time-frequency analysis [28.

The modulation spaces were introduced by Feichtinger [24] in the early eighties. The groundwork theory regarding these spaces of time-frequency analysis was then established in joint collaboration with Gröchenig [25, 26]. The modulation spaces arise from a uniform partition of the frequency space, commonly known as the Wiener decomposition [61]: $\mathbb{R}^{d}=\bigcup_{n \in \mathbb{Z}^{d}} Q_{n}$, where $Q_{n}$ is the unit cube centered at $n \in \mathbb{Z}^{d}$. The Wiener decomposition of $\mathbb{R}^{d}$ induces a natural uniform decomposition of the frequency space of a signal via the (nonsmooth) frequency-uniform decomposition operators $\mathcal{F}^{-1} \chi_{Q_{n}} \mathcal{F}$. Here, $\mathcal{F} u=\widehat{u}$ denotes the Fourier transform of a distribution $u$. The drawback of this approach is the roughness of the characteristic functions $\chi_{Q_{n}}$, but this issue can easily be fixed by smoothing them out appropriately. We have the following definition of the (weighted) modulation spaces $M_{s}^{p, q}$. Let $\psi \in \mathcal{S}\left(\mathbb{R}^{d}\right)$ such that

$$
\operatorname{supp} \psi \subset[-1,1]^{d} \quad \text { and } \quad \sum_{n \in \mathbb{Z}^{d}} \psi(\xi-n) \equiv 1 \text { for any } \xi \in \mathbb{R}^{d} .
$$

Let $0<p, q \leq \infty$ and $s \in \mathbb{R} ; M_{s}^{p, q}$ consists of all tempered distributions $u \in \mathcal{S}^{\prime}\left(\mathbb{R}^{d}\right)$ for which the (quasi) norm

$$
\|u\|_{M_{s}^{p, q}\left(\mathbb{R}^{d}\right)}:=\left\|\langle n\rangle^{s}\right\| \psi(D-n) u\left\|_{L_{x}^{p}\left(\mathbb{R}^{d}\right)}\right\|_{\ell_{n}^{q}\left(\mathbb{Z}^{d}\right)}
$$

is finite. Note that $\psi(D-n) u(x)=\int_{\mathbb{R}^{d}} \psi(\xi-n) \widehat{u}(\xi) e^{2 \pi i x \cdot \xi} d \xi$ is just a Fourier multiplier operator with symbol $\chi_{Q_{n}}$ conveniently smoothed.

It is worthwhile to compare the definition (1.7) with that of the Besov spaces. Let $\varphi_{0}, \varphi \in \mathcal{S}\left(\mathbb{R}^{d}\right)$ such that $\operatorname{supp} \varphi_{0} \subset\{|\xi| \leq 2\}$, $\operatorname{supp} \varphi \subset\left\{\frac{1}{2} \leq|\xi| \leq 2\right\}$, and $\varphi_{0}(\xi)+\sum_{j=1}^{\infty} \varphi\left(2^{-j} \xi\right) \equiv 1$. With $\varphi_{j}(\xi)=\varphi\left(2^{-j} \xi\right)$, we define the (inhomogeneous) Besov spaces $B_{s}^{p, q}$ via the norm

$$
\|u\|_{B_{p, q}^{s}\left(\mathbb{R}^{d}\right)}=\left\|2^{j s}\right\| \varphi_{j}(D) u\left\|_{L^{p}\left(\mathbb{R}^{d}\right)}\right\|_{\ell_{j}^{q}\left(\mathbb{Z}_{\geq 0}\right)} .
$$

There are several known embeddings between Besov, Sobolev, and modulation spaces. See, for example, Okoudjou [47], Toft [59], Sugimoto-Tomita [55], and Kobayashi-Sugimoto [40]. 
Now, given a function $\phi$ on $\mathbb{R}^{d}$, we have

$$
\phi=\sum_{n \in \mathbb{Z}^{d}} \psi(D-n) \phi
$$

where $\psi(D-n)$ is defined above. This decomposition leads to a randomization of $\phi$ that is very natural from the perspective of time-frequency analysis associated to modulation spaces. Let $\left\{g_{n}\right\}_{n \in \mathbb{Z}^{d}}$ be a sequence of independent mean zero complex-valued random variables on a probability space $(\Omega, \mathcal{F}, P)$, where the real and imaginary parts of $g_{n}$ are independent and endowed with probability distributions $\mu_{n}^{(1)}$ and $\mu_{n}^{(2)}$. Then, we can define the Wiener randomization of $\phi$ by

$$
\phi^{\omega}:=\sum_{n \in \mathbb{Z}^{d}} g_{n}(\omega) \psi(D-n) \phi .
$$

Almost simultaneously with our first paper [4, Lührmann-Mendelson [42] also considered a randomization of the form (1.9) (with cubes $Q_{n}$ being substituted by appropriately localized balls) in the study of NLW on $\mathbb{R}^{3}$. See Remark 1.6 below. For a similar randomization used in the study of the Navier-Stokes equations, see the work of Zhang and Fang [63. We would like to stress again, however, that our reason for considering the randomization of the form (1.9) comes from its connection to time-frequency analysis. See also our previous papers [3] and [4].

In the sequel, we make the following assumption on the distributions $\mu_{n}^{(j)}$ : there exists $c>0$ such that

$$
\left|\int_{\mathbb{R}} e^{\gamma x} d \mu_{n}^{(j)}(x)\right| \leq e^{c \gamma^{2}}
$$

for all $\gamma \in \mathbb{R}, n \in \mathbb{Z}^{d}, j=1,2$. Note that (1.10) is satisfied by standard complexvalued Gaussian random variables, standard Bernoulli random variables, and any random variables with compactly supported distributions.

It is easy to see that, if $\phi \in H^{s}\left(\mathbb{R}^{d}\right)$, then the randomized function $\phi^{\omega}$ is almost surely in $H^{s}\left(\mathbb{R}^{d}\right)$. While there is no smoothing upon randomization in terms of differentiability in general, this randomization behaves better under integrability; if $\phi \in L^{2}\left(\mathbb{R}^{d}\right)$, then the randomized function $\phi^{\omega}$ is almost surely in $L^{p}\left(\mathbb{R}^{d}\right)$ for any finite $p \geq 2$. As a result of this enhanced integrability, we have improvements of the Strichartz estimates. See Lemmata 2.2 and 2.3. These improved Strichartz estimates play an essential role in proving probabilistic well-posedness results, which we describe below.

1.3. Main results. Recall that the scaling-critical Sobolev index for the cubic NLS on $\mathbb{R}^{d}$ is $s_{\text {crit }}=\frac{d-2}{2}$. In the following, we take $\phi \in H^{s}\left(\mathbb{R}^{d}\right) \backslash H^{s_{\text {crit }}}\left(\mathbb{R}^{d}\right)$ for some range of $s<s_{\text {crit }}$, that is, below the critical regularity. Then, we consider the wellposedness problem of (1.1) with respect to the randomized initial data $\phi^{\omega}$ defined in (1.9).

For $d \geq 3$, define $s_{d}$ by

$$
s_{d}:=\frac{d-1}{d+1} \cdot s_{\text {crit }}=\frac{d-1}{d+1} \cdot \frac{d-2}{2} .
$$

Note that $s_{d}<s_{\text {crit }}$ and $\frac{s_{d}}{s_{\text {crit }}} \rightarrow 1$ as $d \rightarrow \infty$. Throughout the paper, we use $S(t)=e^{i t \Delta}$ to denote the linear propagator of the Schrödinger group. 
We are now ready to state our main results.

Theorem 1.1 (Almost sure local well-posedness). Let $d \geq 3$ and $s>s_{d}$. Given $\phi \in H^{s}\left(\mathbb{R}^{d}\right)$, let $\phi^{\omega}$ be its Wiener randomization defined in (1.9), satisfying (1.10). Then, the cubic NLS (1.1) on $\mathbb{R}^{d}$ is almost surely locally well-posed with respect to the randomization $\phi^{\omega}$ as initial data. More precisely, there exist $C, c, \gamma>0$ such that for each $0<T \ll 1$, there exists a set $\Omega_{T} \subset \Omega$ with the following properties:

(i) $P\left(\Omega_{T}^{c}\right)<C \exp \left(-\frac{c}{T^{\gamma}\|\phi\|_{H^{s}}^{2}}\right)$.

(ii) For each $\omega \in \Omega_{T}$, there exists a (unique) solution $u$ to (1.1) with $\left.u\right|_{t=0}=\phi^{\omega}$ in the class

$$
S(t) \phi^{\omega}+C\left([-T, T]: H^{\frac{d-2}{2}}\left(\mathbb{R}^{d}\right)\right) \subset C\left([-T, T]: H^{s}\left(\mathbb{R}^{d}\right)\right) .
$$

We prove Theorem 1.1 by considering the equation satisfied by the nonlinear part of a solution $u$. Namely, let $z(t)=z^{\omega}(t):=S(t) \phi^{\omega}$ and $v(t):=u(t)-S(t) \phi^{\omega}$ be the linear and nonlinear parts of $u$, respectively. Then, (1.1) is equivalent to the following perturbed NLS:

$$
\left\{\begin{array}{l}
i \partial_{t} v+\Delta v= \pm|v+z|^{2}(v+z), \\
\left.v\right|_{t=0}=0
\end{array}\right.
$$

We reduce our analysis to the Cauchy problem (1.12) for $v$, viewing $z$ as a random forcing term. Note that such a point of view is common in the study of stochastic PDEs. As a result, the uniqueness in Theorem 1.1 refers to uniqueness of the nonlinear part $v(t)=u(t)-S(t) \phi^{\omega}$ of a solution $u$.

The proof of Theorem 1.1 is based on the fixed point argument involving the variants of the $X^{s, b}$-spaces adapted to the $U^{p}$ - and $V^{p}$-spaces introduced by Koch, Tataru, and their collaborators [29, 30, 41]. See Section 3 for the basic definitions and properties of these function spaces. The main ingredient is the local-in-time improvement of the Strichartz estimates (Lemma 2.2) and the refinement of the bilinear Strichartz estimate (Lemma 3.5 (ii)). We point out that, although $\phi$ and its randomization $\phi^{\omega}$ have a supercritical Sobolev regularity, the randomization essentially makes the problem subcritical, at least locally in time, and therefore, one can also prove Theorem 1.1 only with the classical subcritical $X^{s, b}$-spaces, $b>\frac{1}{2}$. See [4] for the result when $d=4$.

Next, we turn our attention to the global-in-time behavior of the solutions constructed in Theorem 1.1. The key nonlinear estimate in the proof of Theorem 1.1 combined with the global-in-time improvement of the Strichartz estimates (Lemma 2.3) yields the following result on small data global well-posedness and scattering.

Theorem 1.2 (Probabilistic small data global well-posedness and scattering). Let $d \geq 3$ and $s \in\left(s_{d}, s_{\text {crit }}\right]$, where $s_{d}$ is as in (1.11). Given $\phi \in H^{s}\left(\mathbb{R}^{d}\right)$, let $\phi^{\omega}$ be its Wiener randomization defined in (1.9), satisfying (1.10). Then, there exist $C, c>0$ such that for each $0<\varepsilon \ll 1$, there exists a set $\Omega_{\varepsilon} \subset \Omega$ with the following properties:

(i) $P\left(\Omega_{\varepsilon}^{c}\right) \leq C \exp \left(-\frac{c}{\varepsilon^{2}\|\phi\|_{H^{s}}^{2}}\right) \rightarrow 0$ as $\varepsilon \rightarrow 0$.

(ii) For each $\omega \in \Omega_{\varepsilon}$, there exists a (unique) global-in-time solution $u$ to (1.1) with

in the class

$$
\left.u\right|_{t=0}=\varepsilon \phi^{\omega}
$$

$$
\varepsilon S(t) \phi^{\omega}+C\left(\mathbb{R}: H^{\frac{d-2}{2}}\left(\mathbb{R}^{d}\right)\right) \subset C\left(\mathbb{R}: H^{s}\left(\mathbb{R}^{d}\right)\right) .
$$


(iii) We have scattering for each $\omega \in \Omega_{\varepsilon}$. More precisely, for each $\omega \in \Omega_{\varepsilon}$, there exists $v_{+}^{\omega} \in H^{\frac{d-2}{2}}\left(\mathbb{R}^{d}\right)$ such that

$$
\left\|u(t)-S(t)\left(\varepsilon \phi^{\omega}+v_{+}^{\omega}\right)\right\|_{H^{\frac{d-2}{2}}\left(\mathbb{R}^{d}\right)} \rightarrow 0
$$

as $t \rightarrow \infty$. A similar statement holds for $t \rightarrow-\infty$.

In general, a local well-posedness result in a critical space is often accompanied by small data global well-posedness and scattering. In this sense, Theorem 1.2 is an expected consequence of Theorem 1.1 since, in our construction, the nonlinear part $v$ lies in the critical space $H^{\frac{d-2}{2}}\left(\mathbb{R}^{d}\right)$. The next natural question is probabilistic global well-posedness for large data. In order to state our result, we need to make several hypotheses. The first hypothesis is on a probabilistic a priori energy bound on the nonlinear part $v$.

Hypothesis (A). Given any $T, \varepsilon>0$, there exist $R=R(T, \varepsilon)$ and $\Omega_{T, \varepsilon} \subset \Omega$ such that

(i) $P\left(\Omega_{T, \varepsilon}^{c}\right)<\varepsilon$, and

(ii) if $v=v^{\omega}$ is the solution to (1.12) for $\omega \in \Omega_{T, \varepsilon}$, then the following a priori energy estimate holds:

$$
\|v(t)\|_{L^{\infty}\left([0, T] ; H^{\frac{d-2}{2}}\left(\mathbb{R}^{d}\right)\right)} \leq R(T, \varepsilon) .
$$

Note that Hypothesis (A) does not refer to existence of a solution $v=v^{\omega}$ on $[0, T]$ for given $\omega \in \Omega_{T, \varepsilon}$. It only hypothesizes the a priori energy bound (1.13), just like the usual conservation laws. It may be possible to prove (1.13) independently from the argument presented in this paper. Such a probabilistic a priori energy estimate is known, for example, for the cubic NLW. See Burq-Tzvetkov [15]. We point out that the upper bound $R(T, \varepsilon)$ in [15] tends to $\infty$ as $T \rightarrow \infty$. See also 50].

The next hypothesis is on global existence and space-time bounds of solutions to the cubic NLS (1.1) with deterministic initial data belonging to the critical space $H^{\frac{d-2}{2}}\left(\mathbb{R}^{d}\right)$.

Hypothesis (B). Given any $w_{0} \in H^{\frac{d-2}{2}}\left(\mathbb{R}^{d}\right)$, there exists a global solution $w$ to the defocusing cubic NLS (1.1) with $\left.w\right|_{t=0}=w_{0}$. Moreover, there exists a function $C:[0, \infty) \times[0, \infty) \rightarrow[0, \infty)$ which is nondecreasing in each argument such that

$$
\|w\|_{L_{t, x}^{d+2}\left([0, T] \times \mathbb{R}^{d}\right)} \leq C\left(\left\|w_{0}\right\|_{H^{\frac{d-2}{2}}\left(\mathbb{R}^{d}\right)}, T\right)
$$

for any $T>0$.

Note that when $d=4$, Hypothesis (B) is known to be true for any $T>0$ thanks to the global well-posedness result by Ryckman-Vişan [53] and Vişan 60]. For other dimensions $d \geq 3$ with $d \neq 4$, it is not known whether Hypothesis (B) holds. Let us compare (1.14) and the results in [35] and [39. Assuming that $w \in L_{t}^{\infty} \dot{H}_{x}^{s_{\text {crit }}}\left(I_{*} \times \mathbb{R}^{d}\right)$, where $I_{*}$ is a maximal interval of existence, it was shown in [35] and [39] that $I_{*}=\mathbb{R}$ and

$$
\|w\|_{L_{t, x}^{d+2}\left(\mathbb{R} \times \mathbb{R}^{d}\right)} \leq C\left(\|w\|_{L_{t}^{\infty} \dot{H}_{x}^{\frac{d-2}{2}}}{ }_{\left(\mathbb{R} \times \mathbb{R}^{d}\right)}\right) .
$$

We point out that Hypothesis (B) is not directly comparable to the results in 35,39 in the following sense. On the one hand, by assuming that $w \in L_{t}^{\infty} \dot{H}_{x}^{s_{\text {crit }}}\left(I_{*} \times \mathbb{R}^{d}\right)$, 
the results in [35, 39] yield the global-in-time bound (1.15), while Hypothesis (B) assumes the bound (1.14) only for each finite time $T>0$ and does not assume a global-in-time bound. On the other hand, (1.14) is much stronger than (1.15) in the sense that the right-hand side of (1.14) depends only on the size of an initial condition $w_{0}$, while the right-hand side of (1.15) depends on the global-in-time $L_{t}^{\infty} \dot{H}_{x}^{\frac{d-2}{2}}$-bound of the solution $w$. Hypothesis (B), just like Hypothesis (A), is of independent interest from Theorem 1.3 below and is closely related to the fundamental open problem of global well-posedness and scattering for the defocusing cubic NLS (1.1) for $d=3$ and $d \geq 5$.

We now state our third theorem on almost sure global well-posedness of the cubic NLS under Hypotheses (A) and (B). We restrict ourselves to the defocusing NLS in the next theorem.

Theorem 1.3 (Conditional almost sure global well-posedness). Let $d \geq 3$ and $s \in$ $\left(s_{d}, s_{\text {crit }}\right]$, where $s_{d}$ is as in (1.11). Assume Hypothesis (A). Furthermore, assume Hypothesis $(\mathrm{B})$ if $d \neq 4$. Given $\phi \in H^{s}\left(\mathbb{R}^{d}\right)$, let $\phi^{\omega}$ be its Wiener randomization defined in (1.9), satisfying (1.10). Then, the defocusing cubic NLS (1.1) on $\mathbb{R}^{d}$ is almost surely globally well-posed with respect to the randomization $\phi^{\omega}$ as initial data. More precisely, there exists a set $\Sigma \subset \Omega$ with $P(\Sigma)=1$ such that, for each $\omega \in \Sigma$, there exists a (unique) global-in-time solution $u$ to (1.1) with $\left.u\right|_{t=0}=\phi^{\omega}$ in the class

$$
S(t) \phi^{\omega}+C\left(\mathbb{R}: H^{\frac{d-2}{2}}\left(\mathbb{R}^{d}\right)\right) \subset C\left(\mathbb{R}: H^{s}\left(\mathbb{R}^{d}\right)\right) .
$$

The main tool in the proof of Theorem 1.3 is a perturbation lemma for the cubic NLS (Lemma 7.1). Assuming a control on the critical norm (Hypothesis (A)), we iteratively apply the perturbation lemma in the probabilistic setting to show that a solution can be extended to a time depending only on the critical norm. Such a perturbative approach was previously used by Tao-Vişan-Zhang [57] and Killip-Vişan with the second and third authors [37. The novelty of Theorem 1.3 is an application of such a technique in the probabilistic setting. While there is no invariant measure for the nonlinear evolution in our setting, we exploit the quasiinvariance property of the distribution of the linear solution $S(t) \phi^{\omega}$. See Remark 8.2. Our implementation of the proof of Theorem 1.3 is sufficiently general that it can be easily applied to other equations. See [50] in the context of the energy-critical NLW on $\mathbb{R}^{d}, d=4,5$, where both Hypotheses (A) and (B) are satisfied.

When $d \neq 4$, the conditional almost sure global well-posedness in Theorem 1.3 has a flavor analogous to the deterministic conditional global well-posedness in the critical Sobolev spaces by Kenig-Merle [35] and Killip-Vişan [39]. In the following, let us discuss the situation when $d=4$. In this case, we only assume Hypothesis (A) for Theorem 1.3. While it would be interesting to remove this assumption, we do not know how to prove the validity of Hypothesis (A) at this point. This is mainly due to the lack of conservation of $H[v](t)$, i.e. the Hamiltonian evaluated at the nonlinear part $v$ of a solution. In the context of the energy-critical defocusing cubic NLW on $\mathbb{R}^{4}$, however, one can prove an analogue of Hypothesis (A) by establishing a probabilistic a priori bound on the energy $\mathcal{E}[v]$ of the nonlinear part $v$ of a solution, where the energy $\mathcal{E}[v]$ is defined by

$$
\mathcal{E}[v](t)=\frac{1}{2} \int_{\mathbb{R}^{4}}\left|\partial_{t} v(t, x)\right|^{2} d x+\frac{1}{2} \int_{\mathbb{R}^{4}}|\nabla v(t, x)|^{2} d x+\frac{1}{4} \int_{\mathbb{R}^{4}}|v(t, x)|^{4} d x .
$$


As a consequence, the third author [50] successfully implemented a probabilistic perturbation argument and proved almost sure global well-posedness of the energycritical defocusing cubic NLW on $\mathbb{R}^{4}$ with randomized initial data below the scalingcritical regularity 2 We point out that the first term in the energy $\mathcal{E}[v]$ involving the time derivative plays an essential role in establishing a probabilistic a priori bound on the energy for NLW. It seems substantially harder to verify Hypothesis (A) for NLS, even when $d=4$.

While Theorem 1.3 provides only conditional almost sure global existence, our last theorem (Theorem [1.4) below presents a way to construct global-in-time solutions below the scaling-critical regularity with a large probability. The main idea is to use the scaling (1.2) of the equation for random initial data below the scaling criticality. For example, suppose that we have a solution $u$ to (1.1) on a short time interval with a deterministic initial condition $u_{0} \in H^{s}\left(\mathbb{R}^{d}\right), s<s_{\text {crit }}$. In view of (1.2) and (1.3), by taking $\mu \rightarrow 0$, we see that the $H^{s}$-norm of the scaled initial condition goes to 0 . Thus, one might think that the problem can be reduced to small data theory. This, of course, does not work in the usual deterministic setting, since we do not know how to construct solutions depending only on the $H^{s}$-norm of the initial data, $s<s_{\text {crit }}$. Even in the probabilistic setting, this naive idea does not work if we simply apply the scaling to the randomized function $\phi^{\omega}$ defined in (1.9). This is due to the fact that we need to use (sub)critical space-time norms controlling the random linear term $z^{\omega}(t)=S(t) \phi^{\omega}$, which do not become small even if we take $\mu \ll 1$.

To resolve this issue, we consider a randomization based on a partition of the frequency space by dilated cubes. Given $\mu>0$, define $\psi^{\mu}$ by

$$
\psi^{\mu}(\xi)=\psi\left(\mu^{-1} \xi\right) \text {. }
$$

Then, we can write a function $\phi$ on $\mathbb{R}^{d}$ as

$$
\phi=\sum_{n \in \mathbb{Z}^{d}} \psi^{\mu}(D-\mu n) \phi .
$$

Now, we introduce the randomization $\phi^{\omega, \mu}$ of $\phi$ on dilated cubes of scale $\mu$ by

$$
\phi^{\omega, \mu}:=\sum_{n \in \mathbb{Z}^{d}} g_{n}(\omega) \psi^{\mu}(D-\mu n) \phi
$$

where $\left\{g_{n}\right\}_{n \in \mathbb{Z}^{d}}$ is a sequence of independent mean zero complex-valued random variables, satisfying (1.10) as before. Then, we have the following global wellposedness of (1.1) with a large probability.

Theorem 1.4. Let $d \geq 3$ and $\phi \in H^{s}\left(\mathbb{R}^{d}\right)$, for some $s \in\left(s_{d}, s_{\text {crit }}\right)$, where $s_{d}$ is as in (1.11). Then, given the randomization $\phi^{\omega, \mu}$ on dilated cubes of scale $\mu \ll 1$ defined in (1.17), satisfying (1.10), the cubic NLS (1.1) on $\mathbb{R}^{d}$ is globally well-posed with a large probability. More precisely, for each $0<\varepsilon \ll 1$, there exists a small dilation scale $\mu_{0}=\mu_{0}\left(\varepsilon,\|\phi\|_{H^{s}}\right)>0$ such that for each $\mu \in\left(0, \mu_{0}\right)$, there exists a set $\Omega_{\mu} \subset \Omega$ with the following properties:

(i) $P\left(\Omega_{\mu}^{c}\right)<\varepsilon$.

\footnotetext{
${ }^{2}$ In [50], the third author also proved almost sure global well-posedness of the energy-critical defocusing NLW on $\mathbb{R}^{5}$. This result was recently extended to dimension 3 by the second and third authors [46].
} 
(ii) If $\phi^{\omega, \mu}$ is the randomization on dilated cubes defined in (1.17), satisfying (1.10), then, for each $\omega \in \Omega_{\mu}$, there exists a (unique) global-in-time solution $u$ to (1.1) with $\left.u\right|_{t=0}=\phi^{\omega, \mu}$ in the class

$$
S(t) \phi^{\omega}+C\left(\mathbb{R}: H^{\frac{d-2}{2}}\left(\mathbb{R}^{d}\right)\right) \subset C\left(\mathbb{R}: H^{s}\left(\mathbb{R}^{d}\right)\right) .
$$

Moreover, for each $\omega \in \Omega_{\mu}$, scattering holds in the sense that there exists $v_{+}^{\omega} \in H^{\frac{d-2}{2}}\left(\mathbb{R}^{d}\right)$ such that

$$
\left\|u(t)-S(t)\left(\phi^{\omega, \mu}+v_{+}^{\omega}\right)\right\|_{H^{\frac{d-2}{2}}\left(\mathbb{R}^{d}\right)} \rightarrow 0
$$

as $t \rightarrow \infty$. A similar statement holds for $t \rightarrow-\infty$.

We conclude this introduction with several remarks.

Remark 1.5. In probabilistic well-posedness results [6, 7, 20, 44] for NLS on $\mathbb{T}^{d}$, random initial data are assumed to be of the following specific form:

$$
u_{0}^{\omega}(x)=\sum_{n \in \mathbb{Z}^{d}} \frac{g_{n}(\omega)}{\left(1+|n|^{2}\right)^{\frac{\alpha}{2}}} e^{i n \cdot x},
$$

where $\left\{g_{n}\right\}_{n \in \mathbb{Z}^{d}}$ is a sequence of independent complex-valued standard Gaussian random variables. The expression (1.18) has a close connection to the study of invariant measures and hence it is of importance. At the same time, due to the lack of a full range of Strichartz estimates on $\mathbb{T}^{d}$, one could not handle a general randomization of a given function as in (1.5). In this paper, we consider NLS on $\mathbb{R}^{d}$ and thus we do not encounter this issue thanks to a full range of the Strichartz estimates. For NLW, finite speed of propagation allows us to use a full range of Strichartz estimates even on compact domains, at least locally in time. Thus, one does not encounter such an issue.

Remark 1.6. In a recent preprint, Lührmann-Mendelson [42] considered the defocusing NLW on $\mathbb{R}^{3}$ with randomized initial data, essentially given by (1.9), below the critical regularity and proved almost sure global well-posedness in the energysubcritical case, following the method developed in 20, namely an adaptation of Bourgain's high-low method [8] in the probabilistic setting. As Bourgain's high-low method is a subcritical tool, their global result misses the energy-critical case 3

The third author [50] recently proved almost sure global well-posedness of the energy-critical defocusing NLW on $\mathbb{R}^{d}, d=4,5$, with randomized initial data below the critical regularity. The argument is based on an application of a perturbation lemma as in Theorem 1.3 along with a probabilistic a priori control on the energy, which is not available for the cubic NLS (1.1).

This paper is organized as follows. In Section 2, we state some probabilistic lemmata. In Section 3, we go over the basic definitions and properties of function spaces involving the $U^{p}$ - and $V^{p}$-spaces. We prove the key nonlinear estimates in Section 4 and then use them to prove Theorems 1.1 and 1.2 in Section 5 . We divide the proof of Theorem 1.3 into three sections. In Sections [6] and 7, we discuss the Cauchy theory for the defocusing cubic NLS with a deterministic perturbation. We implement these results in the probabilistic setting and prove Theorem 1.3 in Section 8 . In Section 9, we show how Theorem 1.4 follows from the arguments in

\footnotetext{
${ }^{3}$ In [46], the second and third authors recently proved almost sure global well-posedness of the energy-critical defocusing quintic NLW on $\mathbb{R}^{3}$.
} 
Sections 4 and 5, once we consider a randomization on dilated cubes. In Appendix A, we state and prove some additional properties of the function spaces defined in Section 3 .

Lastly, note that we present the proofs of these results only for positive times in view of the time reversibility of (1.1).

\section{Probabilistic lemmata}

In this section, we summarize the probabilistic lemmata used in this paper. In particular, the probabilistic Strichartz estimates (Lemmata 2.2 and 2.3) play an essential role. First, we recall the usual Strichartz estimates on $\mathbb{R}^{d}$ for the readers' convenience. We say that a pair $(q, r)$ is Schrödinger admissible if it satisfies

$$
\frac{2}{q}+\frac{d}{r}=\frac{d}{2}
$$

with $2 \leq q, r \leq \infty$ and $(q, r, d) \neq(2, \infty, 2)$. Then, the following Strichartz estimates are known to hold.

Lemma 2.1 (27, 33, 54,62]). Let $(q, r)$ be Schrödinger admissible. Then, we have

$$
\|S(t) \phi\|_{L_{t}^{q} L_{x}^{r}\left(\mathbb{R} \times \mathbb{R}^{d}\right)} \lesssim\|\phi\|_{L_{x}^{2}\left(\mathbb{R}^{d}\right)} .
$$

In particular, when $q=r$, we have $q=r=\frac{2(d+2)}{d}$. By applying Sobolev inequality and (2.2), we also have

$$
\|S(t) \phi\|_{L_{t, x}^{p}\left(\mathbb{R} \times \mathbb{R}^{d}\right)} \lesssim\left\||\nabla|^{\frac{d}{2}-\frac{d+2}{p}} \phi\right\|_{L_{x}^{2}\left(\mathbb{R}^{d}\right)}
$$

for $p \geq \frac{2(d+2)}{d}$. Recall that the derivative loss in (2.3) depends only on the size of the frequency support and not its location. Namely, if $\widehat{\phi}$ is supported on a cube $Q$ of side length $N$, then we have

$$
\|S(t) \phi\|_{L_{t, x}^{p}\left(\mathbb{R} \times \mathbb{R}^{d}\right)} \lesssim N^{\frac{d}{2}-\frac{d+2}{p}}\|\phi\|_{L_{x}^{2}\left(\mathbb{R}^{d}\right)},
$$

regardless of the center of the cube $Q$.

Next, we present improvements of the Strichartz estimates under the Wiener randomization (1.9) and where, throughout, we assume (1.10). See 4 for the proofs.

Lemma 2.2 (Improved local-in-time Strichartz estimate). Given $\phi \in L^{2}\left(\mathbb{R}^{d}\right)$, let $\phi^{\omega}$ be its Wiener randomization defined in (1.9), satisfying (1.10). Then, given finite $q, r \geq 2$, there exist $C, c>0$ such that

$$
P\left(\left\|S(t) \phi^{\omega}\right\|_{L_{t}^{q} L_{x}^{r}\left([0, T] \times \mathbb{R}^{d}\right)}>\lambda\right) \leq C \exp \left(-c \frac{\lambda^{2}}{T^{\frac{2}{q}}\|\phi\|_{L^{2}}^{2}}\right)
$$

for all $T>0$ and $\lambda>0$. In particular, with $\lambda=T^{\theta}$, we have

$$
\left\|S(t) \phi^{\omega}\right\|_{L_{t}^{q} L_{x}^{r}\left([0, T] \times \mathbb{R}^{d}\right)} \lesssim T^{\theta}
$$

outside a set of probability

$$
\leq C \exp \left(-c \frac{1}{T^{2\left(\frac{1}{q}-\theta\right)}\|\phi\|_{L^{2}}^{2}}\right) .
$$

Note that this probability can be made arbitrarily small by letting $T \rightarrow 0$ as long as $\theta<\frac{1}{q}$. 
The next lemma states an improvement of the Strichartz estimates in the globalin-time setting.

Lemma 2.3 (Improved global-in-time Strichartz estimate). Given $\phi \in L^{2}\left(\mathbb{R}^{d}\right)$, let $\phi^{\omega}$ be its Wiener randomization defined in (1.9), satisfying (1.10). Given a Schrödinger admissible pair $(q, r)$ with $q, r<\infty$, let $\widetilde{r} \geq r$. Then, there exist $C, c>0$ such that

$$
P\left(\left\|S(t) \phi^{\omega}\right\|_{L_{t}^{q} L_{x}^{\tilde{r}}\left(\mathbb{R} \times \mathbb{R}^{d}\right)}>\lambda\right) \leq C e^{-c \lambda^{2}\|\phi\|_{L^{2}}^{-2}} .
$$

In particular, given any small $\varepsilon>0$, we have

$$
\left\|S(t) \phi^{\omega}\right\|_{L_{t}^{q} L_{x}^{\tilde{r}}\left(\mathbb{R} \times \mathbb{R}^{d}\right)} \lesssim\left(\log \frac{1}{\varepsilon}\right)^{\frac{1}{2}}\|\phi\|_{L^{2}\left(\mathbb{R}^{d}\right)}
$$

outside a set of probability $<\varepsilon$.

Recall that the diagonal Strichartz admissible index is given by $p=\frac{2(d+2)}{d}$. In the diagonal case $q=\widetilde{r}$, it is easy to see that the condition of Lemma 2.3 is satisfied if $q=\widetilde{r} \geq p=\frac{2(d+2)}{d}$. In the following, we apply Lemma 2.3 in this setting.

We also need the following lemma on the control of the size of $H^{s}$-norm of $\phi^{\omega}$.

Lemma 2.4. Given $\phi \in H^{s}\left(\mathbb{R}^{d}\right)$, let $\phi^{\omega}$ be its Wiener randomization defined in (1.9), satisfying (1.10). Then, we have

$$
P\left(\left\|\phi^{\omega}\right\|_{H^{s}\left(\mathbb{R}^{d}\right)}>\lambda\right) \leq C e^{-c \lambda^{2}\|\phi\|_{H^{s}}^{-2}} .
$$

We conclude this section by introducing some notation involving Strichartz and space-time Lebesgue spaces. In the sequel, given an interval $I \subset \mathbb{R}$, we often use $L_{t}^{q} L_{x}^{r}(I)$ to denote $L_{t}^{q} L_{x}^{r}\left(I \times \mathbb{R}^{d}\right)$. We also define the $\dot{S}^{s_{\text {crit }}}(I)$-norm in the usual manner by setting

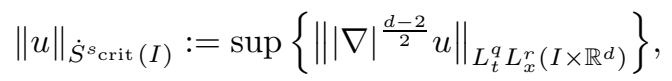

where the supremum is taken over all Schrödinger admissible pairs $(q, r)$.

\section{Function spaces And their properties}

In this section, we go over the basic definitions and properties of the $U^{p}$ - and $V^{p}$-spaces, developed by Tataru, Koch, and their collaborators [29, 30, 41]. These spaces have been very effective in establishing well-posedness of various dispersive PDEs in critical regularities. See Hadac-Herr-Koch [29] and Herr-Tataru-Tzvetkov [30] for detailed proofs.

Let $H$ be a separable Hilbert space over $\mathbb{C}$. In particular, it will be either $H^{s}\left(\mathbb{R}^{d}\right)$ or $\mathbb{C}$. Let $\mathcal{Z}$ be the collection of finite partitions $\left\{t_{k}\right\}_{k=0}^{K}$ of $\mathbb{R}:-\infty<t_{0}<\cdots<$ $t_{K} \leq \infty$. If $t_{K}=\infty$, we use the convention $u\left(t_{K}\right):=0$ for all functions $u: \mathbb{R} \rightarrow H$. We use $\chi_{I}$ to denote the sharp characteristic function of a set $I \subset \mathbb{R}$.

Definition 3.1. Let $1 \leq p<\infty$.

(i) A $U^{p}$-atom is defined by a step function $a: \mathbb{R} \rightarrow H$ of the form

$$
a=\sum_{k=1}^{K} \phi_{k-1} \chi_{\left[t_{k-1}, t_{k}\right)},
$$


where $\left\{t_{k}\right\}_{k=0}^{K} \in \mathcal{Z}$ and $\left\{\phi_{k}\right\}_{k=0}^{K-1} \subset H$ with $\sum_{k=0}^{K-1}\left\|\phi_{k}\right\|_{H}^{p}=1$. Then, we define the atomic space $U^{p}(\mathbb{R} ; H)$ to be the collection of functions $u: \mathbb{R} \rightarrow H$ of the form

$$
u=\sum_{j=1}^{\infty} \lambda_{j} a_{j}, \quad \text { where } a_{j} \text { 's are } U^{p} \text {-atoms and }\left\{\lambda_{j}\right\}_{j \in \mathbb{N}} \in \ell^{1}(\mathbb{N} ; \mathbb{C}),
$$

with the norm

$$
\|u\|_{U^{p}(\mathbb{R} ; H)}:=\inf \left\{\|\lambda\|_{\ell^{1}}: \text { (3.1) holds with } \lambda=\left\{\lambda_{j}\right\}_{j \in \mathbb{N}} \text { and some } U^{p} \text {-atoms } a_{j}\right\} \text {. }
$$

(ii) We define the space $V^{p}(\mathbb{R} ; H)$ of functions of bounded $p$-variation to be the collection of functions $u: \mathbb{R} \rightarrow H$ with $\|u\|_{V^{p}(\mathbb{R} ; H)}<\infty$, where the $V^{p}$-norm is defined by

$$
\|u\|_{V^{p}(\mathbb{R} ; H)}:=\sup _{\left\{t_{k}\right\}_{k=0}^{K} \in \mathcal{Z}}\left(\sum_{k=1}^{K}\left\|u\left(t_{k}\right)-u\left(t_{k-1}\right)\right\|_{H}^{p}\right)^{\frac{1}{p}} .
$$

We also define $V_{\mathrm{rc}}^{p}(\mathbb{R} ; H)$ to be the closed subspace of all right-continuous functions in $V^{p}(\mathbb{R} ; H)$ such that $\lim _{t \rightarrow-\infty} u(t)=0$.

(iii) Let $s \in \mathbb{R}$. We define $U_{\Delta}^{p} H^{s}$ (and $V_{\Delta}^{p} H^{s}$, respectively) to be the spaces of all functions $u: \mathbb{R} \rightarrow H^{s}\left(\mathbb{T}^{d}\right)$ such that the following $U_{\Delta}^{p} H^{s}$-norm (and $V_{\Delta}^{p} H^{s}$-norm, respectively) is finite:

$$
\|u\|_{U_{\Delta}^{p} H^{s}}:=\|S(-t) u\|_{U^{p}\left(\mathbb{R} ; H^{s}\right)} \quad \text { and } \quad\|u\|_{V_{\Delta}^{p} H^{s}}:=\|S(-t) u\|_{V^{p}\left(\mathbb{R} ; H^{s}\right)},
$$

where $S(t)=e^{i t \Delta}$ denotes the linear propagator for (1.1). We use $V_{\mathrm{rc}, \Delta}^{p} H^{s}$ to denote the subspace of right-continuous functions in $V_{\Delta}^{p} H^{s}$.

Remark 3.2. Note that the spaces $U^{p}(\mathbb{R} ; H), V^{p}(\mathbb{R} ; H)$, and $V_{\mathrm{rc}}^{p}(\mathbb{R} ; H)$ are Banach spaces. The closed subspace of continuous functions in $U^{p}(\mathbb{R} ; H)$ is also a Banach space. Moreover, we have the following embeddings:

$$
U^{p}(\mathbb{R} ; H) \hookrightarrow V_{\mathrm{rc}}^{p}(\mathbb{R} ; H) \hookrightarrow U^{q}(\mathbb{R} ; H) \hookrightarrow L^{\infty}(\mathbb{R} ; H)
$$

for $1 \leq p<q<\infty$. Similar embeddings hold for $U_{\Delta}^{p} H^{s}$ and $V_{\Delta}^{p} H^{s}$.

Next, we state a transference principle and an interpolation result.

Lemma 3.3. (i) (Transference principle) Suppose that we have

$$
\left\|T\left(S(t) \phi_{1}, \ldots, S(t) \phi_{k}\right)\right\|_{L_{t}^{p} L_{x}^{q}\left(\mathbb{R} \times \mathbb{R}^{d}\right)} \lesssim \prod_{j=1}^{k}\left\|\phi_{j}\right\|_{L_{x}^{2}}
$$

for some $1 \leq p, q \leq \infty$. Then, we have

$$
\left\|T\left(u_{1}, \ldots, u_{k}\right)\right\|_{L_{t}^{p} L_{x}^{q}\left(\mathbb{R} \times \mathbb{R}^{d}\right)} \lesssim \prod_{j=1}^{k}\left\|u_{j}\right\|_{U_{\Delta}^{p} L_{x}^{2}}
$$

(ii) (Interpolation) Let $E$ be a Banach space. Suppose that $T: U^{p_{1}} \times \cdots \times U^{p_{k}} \rightarrow$ $E$ is a bounded $k$-linear operator such that

$$
\left\|T\left(u_{1}, \ldots, u_{k}\right)\right\|_{E} \leq C_{1} \prod_{j=1}^{k}\left\|u_{j}\right\|_{U^{p_{j}}}
$$


for some $p_{1}, \ldots, p_{k}>2$. Moreover, assume that there exists $C_{2} \in\left(0, C_{1}\right]$ such that

$$
\left\|T\left(u_{1}, \ldots, u_{k}\right)\right\|_{E} \leq C_{2} \prod_{j=1}^{k}\left\|u_{j}\right\|_{U^{2}} .
$$

Then, we have

$$
\left\|T\left(u_{1}, \ldots, u_{k}\right)\right\|_{E} \leq C_{2}\left(\ln \frac{C_{1}}{C_{2}}+1\right)^{k} \prod_{j=1}^{k}\left\|u_{j}\right\|_{V^{2}}
$$

for $u_{j} \in V_{\mathrm{rc}}^{2}, j=1, \ldots, k$.

A transference principle as above has been commonly used in the Fourier restriction norm method. See [29, Proposition 2.19] for the proof of Lemma 3.3 (i). The proof of the interpolation result follows from extending the trilinear result in [30] to a general $k$-linear case. See also [29, Proposition 2.20].

Let $\eta: \mathbb{R} \rightarrow[0,1]$ be an even, smooth cutoff function supported on $\left[-\frac{8}{5}, \frac{8}{5}\right]$ such that $\eta \equiv 1$ on $\left[-\frac{5}{4}, \frac{5}{4}\right]$. Given a dyadic number $N \geq 1$, we set $\eta_{1}(\xi)=\eta(|\xi|)$ and

$$
\eta_{N}(\xi)=\eta\left(\frac{|\xi|}{N}\right)-\eta\left(\frac{2|\xi|}{N}\right)
$$

for $N \geq 2$. Then, we define the Littlewood-Paley projection operator $\mathbf{P}_{N}$ as the Fourier multiplier operator with symbol $\eta_{N}$. Moreover, we define $\mathbf{P}_{\leq N}$ and $\mathbf{P}_{\geq N}$ by $\mathbf{P}_{\leq N}=\sum_{1 \leq M \leq N} \mathbf{P}_{M}$ and $\mathbf{P}_{\geq N}=\sum_{M \geq N} \mathbf{P}_{M}$.

Definition 3.4. (i) Let $s \in \mathbb{R}$. We define $X^{s}(\mathbb{R})$ to be the space of all tempered distributions $u: \mathbb{R} \rightarrow H^{s}\left(\mathbb{R}^{d}\right)$ such that $\|u\|_{X^{s}(\mathbb{R})}<\infty$, where the $X^{s}$-norm is defined by

$$
\|u\|_{X^{s}(\mathbb{R})}:=\left(\sum_{\substack{N \geq 1 \\ \text { dyadic }}} N^{2 s}\left\|\mathbf{P}_{N} u\right\|_{U_{\Delta}^{2} L^{2}}^{2}\right)^{\frac{1}{2}} .
$$

(ii) Let $s \in \mathbb{R}$. We define $Y^{s}(\mathbb{R})$ to be the space of all tempered distributions $u: \mathbb{R} \rightarrow H^{s}\left(\mathbb{R}^{d}\right)$ such that for every $N \in \mathbb{N}$, the map $t \mapsto \mathbf{P}_{N} u(t)$ is in $V_{\mathrm{rc}, \Delta}^{2} H^{s}$ and $\|u\|_{Y^{s}(\mathbb{R})}<\infty$, where the $Y^{s}$-norm is defined by

$$
\|u\|_{Y^{s}(\mathbb{R})}:=\left(\sum_{\substack{N \geq 1 \\ \text { dyadic }}} N^{2 s}\left\|\mathbf{P}_{N} u\right\|_{V_{\Delta}^{2} L^{2}}^{2}\right)^{\frac{1}{2}} .
$$

Recall the following embeddings:

$$
U_{\Delta}^{2} H^{s} \hookrightarrow X^{s} \hookrightarrow Y^{s} \hookrightarrow V_{\Delta}^{2} H^{s} \hookrightarrow U_{\Delta}^{p} H^{s}
$$

for $p>2$.

Given an interval $I \subset \mathbb{R}$, we define the local-in-time versions $X^{s}(I)$ and $Y^{s}(I)$ of these spaces as restriction norms. For example, we define the $X^{s}(I)$-norm by

$$
\|u\|_{X^{s}(I)}=\inf \left\{\|v\|_{X^{s}(\mathbb{R})}:\left.v\right|_{I}=u\right\} .
$$

We also define the norm for the nonhomogeneous term:

$$
\|F\|_{N^{s}(I)}=\left\|\int_{t_{0}}^{t} S\left(t-t^{\prime}\right) F\left(t^{\prime}\right) d t^{\prime}\right\|_{X^{s}(I)} .
$$


In the following, we will perform our analysis in $X^{s}(I) \cap C\left(I ; H^{s}\right)$, that is, in a Banach subspace of continuous functions in $X^{s}(I)$. See Appendix $\mathrm{A}$ for additional properties of the $X^{s}(I)$-spaces.

We conclude this section by presenting some basic estimates involving these function spaces.

Lemma 3.5. (i) (Linear estimates) Let $s \geq 0$ and $0<T \leq \infty$. Then, we have

$$
\begin{aligned}
\|S(t) \phi\|_{X^{s}([0, T))} & \leq\|\phi\|_{H^{s}}, \\
\|F\|_{N^{s}([0, T))} & \leq \sup _{\substack{v \in Y^{-s}([0, T)) \\
\|v\|_{Y^{-s}=1}}}\left|\int_{0}^{T} \int_{\mathbb{R}^{d}} F(t, x) \overline{v(t, x)} d x d t\right|
\end{aligned}
$$

for all $\phi \in H^{s}\left(\mathbb{R}^{d}\right)$ and $F \in L^{1}\left([0, T) ; H^{s}\left(\mathbb{R}^{d}\right)\right)$.

(ii) (Strichartz estimates) Let $(q, r)$ be Schrödinger admissible with $q>2$ and $p \geq \frac{2(d+2)}{d}$. Then, for $0<T \leq \infty$ and $N_{1} \leq N_{2}$, we have

$$
\begin{gathered}
\|u\|_{L_{t}^{q} L_{x}^{r}\left([0, T) \times \mathbb{R}^{d}\right)} \lesssim\|u\|_{Y^{0}([0, T))}, \\
\|u\|_{L_{t, x}^{p}\left([0, T) \times \mathbb{R}^{d}\right)} \lesssim\left\||\nabla|^{\frac{d}{2}-\frac{d+2}{p}} u\right\|_{Y^{0}([0, T))}, \\
\left\|\mathbf{P}_{N_{1}} u_{1} \mathbf{P}_{N_{2}} u_{2}\right\|_{L_{t, x}^{2}\left([0, T) \times \mathbb{R}^{d}\right)} \\
\lesssim N_{1}^{\frac{d-2}{2}}\left(\frac{N_{1}}{N_{2}}\right)^{\frac{1}{2}-}\left\|\mathbf{P}_{N_{1}} u_{1}\right\|_{Y^{0}([0, T))}\left\|\mathbf{P}_{N_{2}} u_{2}\right\|_{Y^{0}([0, T))} .
\end{gathered}
$$

Note that there is a slight loss of regularity in (3.6) since we use the $Y^{0}$-norm on the right-hand side instead of the $X^{0}$-norm. In view of (3.2), we may replace the $Y^{0}$-norms on the right-hand sides of (3.4), (3.5), and (3.6) by the $X^{0}$-norm in the following.

Proof. In the following, we briefly discuss the proof of (ii). See [29,30] for the proof of (i). The first estimate (3.4) follows from the Strichartz estimate (2.2), Lemma 3.3 (i), and (3.2):

$$
\|u\|_{L_{t}^{q} L_{x}^{r}} \lesssim\|u\|_{U_{\Delta}^{q} L^{2}} \lesssim\|u\|_{Y^{0}}
$$

for $q>2$. The second estimate (3.5) follows from (2.3) in a similar manner. It remains to prove (3.6). On the one hand, the following bilinear refinement of the Strichartz estimate by Bourgain [8] and Ozawa-Tsutsumi [48]:

$$
\left\|\mathbf{P}_{N_{1}} S(t) \phi_{1} \mathbf{P}_{N_{2}} S(t) \phi_{2}\right\|_{L_{t, x}^{2}} \lesssim N_{1}^{\frac{d-2}{2}}\left(\frac{N_{1}}{N_{2}}\right)^{\frac{1}{2}}\left\|\mathbf{P}_{N_{1}} \phi_{1}\right\|_{L^{2}}\left\|\mathbf{P}_{N_{2}} \phi_{2}\right\|_{L^{2}}
$$

and Lemma 3.3 (i) yield

$$
\left\|\mathbf{P}_{N_{1}} u_{1} \mathbf{P}_{N_{2}} u_{2}\right\|_{L_{t, x}^{2}} \lesssim N_{1}^{\frac{d-2}{2}}\left(\frac{N_{1}}{N_{2}}\right)^{\frac{1}{2}}\left\|\mathbf{P}_{N_{1}} u_{1}\right\|_{U_{\Delta}^{2} L^{2}}\left\|\mathbf{P}_{N_{2}} u_{2}\right\|_{U_{\Delta}^{2} L^{2}} .
$$

On the other hand, by Bernstein's inequality and noting that $\left(4, \frac{2 d}{d-1}\right)$ is Strichartz admissible, we have

$$
\left\|\mathbf{P}_{N_{j}} S(t) \phi_{j}\right\|_{L_{t, x}^{4}} \lesssim N_{j}^{\frac{d-2}{4}}\left\|\mathbf{P}_{N_{j}} S(t) \phi_{j}\right\|_{L_{t}^{4} L_{x}^{\frac{2 d}{d-1}}} \lesssim N_{j}^{\frac{d-2}{4}}\left\|\mathbf{P}_{N_{j}} \phi_{j}\right\|_{L^{2}}
$$


Then, by Cauchy-Schwarz' inequality and Lemma 3.3 (i), we obtain

$$
\left\|\mathbf{P}_{N_{1}} u_{1} \mathbf{P}_{N_{2}} u_{2}\right\|_{L_{t, x}^{2}} \lesssim N_{1}^{\frac{d-2}{4}} N_{2}^{\frac{d-2}{4}}\left\|\mathbf{P}_{N_{1}} u_{1}\right\|_{U_{\Delta}^{4} L^{2}}\left\|\mathbf{P}_{N_{2}} u_{2}\right\|_{U_{\Delta}^{4} L^{2}}
$$

Hence, by Lemma 3.3 (ii), with (3.7) and (3.8), we have

$$
\begin{aligned}
\left\|\mathbf{P}_{N_{1}} u_{1} \mathbf{P}_{N_{2}} u_{2}\right\|_{L_{t, x}^{2}} \\
\quad \lesssim N_{1}^{\frac{d-2}{2}}\left(\frac{N_{1}}{N_{2}}\right)^{\frac{1}{2}}\left(\ln \left(\frac{N_{2}}{N_{1}}\right)+1\right)^{2}\left\|\mathbf{P}_{N_{1}} u_{1}\right\|_{V_{\Delta}^{2} L^{2}}\left\|\mathbf{P}_{N_{2}} u_{2}\right\|_{V_{\Delta}^{2} L^{2}} .
\end{aligned}
$$

Finally, (3.6) follows from (3.2) and (3.9).

Similar to the usual Strichartz estimate (2.3), the derivative loss in (3.5) depends only on the size of the spatial frequency support and not its location. Namely, if the spatial frequency support of $\widehat{u}(t, \xi)$ is contained in a cube of side length $N$ for all $t \in \mathbb{R}$, then we have

$$
\|u\|_{L_{t, x}^{p}\left([0, T) \times \mathbb{R}^{d}\right)} \lesssim N^{\frac{d}{2}-\frac{d+2}{p}}\|u\|_{Y^{0}([0, T))} .
$$

This is a direct consequence of (2.4).

Lastly, we recall Schur's test for the readers' convenience.

Lemma 3.6 (Schur's test). Suppose that we have

$$
\sup _{m} \sum_{n}\left|K_{m, n}\right|+\sup _{n} \sum_{m}\left|K_{m, n}\right|<\infty
$$

for some $K_{m, n} \in \mathbb{C}, m, n \in \mathbb{Z}$. Then, we have

$$
\sum_{m, n} K_{m, n} a_{m} b_{n} \lesssim\left\|a_{m}\right\|_{\ell_{m}^{2}}\left\|b_{n}\right\|_{\ell_{n}^{2}}
$$

for any $\ell^{2}$-sequences $\left\{a_{m}\right\}_{m \in \mathbb{Z}}$ and $\left\{b_{n}\right\}_{n \in \mathbb{Z}}$.

\section{Probabilistic nonlinear estimates}

In this section, we prove the key nonlinear estimates in the critical regularity $s_{\text {crit }}=\frac{d-2}{2}$. In the next section, we use them to prove Theorems 1.1 and 1.2 . Given $z(t)=S(t) \phi^{\omega}$, define $\Gamma$ by

$$
\Gamma v(t)=\mp i \int_{0}^{t} S\left(t-t^{\prime}\right) \mathcal{N}(v+z)\left(t^{\prime}\right) d t^{\prime},
$$

where $\mathcal{N}(v+z)=|v+z|^{2}(v+z)$. Then, we have the following nonlinear estimates.

Proposition 4.1. Given $d \geq 3$, let $s \in\left(s_{d}, s_{\text {crit }}\right]$, where $s_{d}$ is defined in (1.11). Given $\phi \in H^{s}\left(\mathbb{R}^{d}\right)$, let $\phi^{\omega}$ be its Wiener randomization defined in (1.9), satisfying (1.10).

(i) Let $0<T \leq 1$. Then, there exists $0<\theta \ll 1$ such that we have

$$
\begin{aligned}
& \|\Gamma v\|_{X \frac{d-2}{2}([0, T))} \leq C_{1}\left(\|v\|_{X}^{3}{ }_{X-2}^{\frac{d-2}{2}([0, T))}+T^{\theta} R^{3}\right), \\
& \left\|\Gamma v_{1}-\Gamma v_{2}\right\|_{X} \frac{d-2}{2}([0, T)) \\
& \leq C_{2}\left(\sum_{j=1}^{2}\left\|v_{j}\right\|_{X^{\frac{d-2}{2}}([0, T))}^{2}+T^{\theta} R^{2}\right)\left\|v_{1}-v_{2}\right\|_{X^{\frac{d-2}{2}}([0, T))}
\end{aligned}
$$


for all $v, v_{1}, v_{2} \in X^{\frac{d-2}{2}}([0, T))$ and $R>0$, outside a set of probability $\leq C \exp \left(-c \frac{R^{2}}{\|\phi\|_{H^{s}}^{2}}\right)$.

(ii) Given $0<\varepsilon \ll 1$, define $\widetilde{\Gamma}$ by

$$
\widetilde{\Gamma} v(t)=\mp i \int_{0}^{t} S\left(t-t^{\prime}\right) \mathcal{N}(v+\varepsilon z)\left(t^{\prime}\right) d t^{\prime} .
$$

Then, we have

$$
\begin{gathered}
\|\widetilde{\Gamma} v\|_{X \frac{d-2}{2}(\mathbb{R})} \leq C_{3}\left(\|v\|_{X}^{3}{ }_{X-2}^{\frac{d-2}{2}(\mathbb{R})}+R^{3}\right), \\
\left\|\widetilde{\Gamma} v_{1}-\widetilde{\Gamma} v_{2}\right\|_{X \frac{d-2}{2}(\mathbb{R})} \leq C_{4}\left(\sum_{j=1}^{2}\left\|v_{j}\right\|_{X \frac{d-2}{2}(\mathbb{R})}^{2}+R^{2}\right)\left\|v_{1}-v_{2}\right\|_{X^{\frac{d-2}{2}}(\mathbb{R})}
\end{gathered}
$$

for all $v, v_{1}, v_{2} \in X^{\frac{d-2}{2}}(\mathbb{R})$ and $R>0$, outside a set of probability $\leq C \exp \left(-c \frac{R^{2}}{\varepsilon^{2}\|\phi\|_{H^{s}}^{2}}\right)$.

Proof. (i) Let $0<T \leq 1$. We only prove (4.2) since (4.3) follows in a similar manner. Given $N \geq 1$, define $\Gamma_{N}$ by

$$
\Gamma_{N} v(t)=\mp i \int_{0}^{t} S\left(t-t^{\prime}\right) \mathbf{P}_{\leq N} \mathcal{N}(v+z)\left(t^{\prime}\right) d t^{\prime} .
$$

By Bernstein's and Hölder's inequalities, we have

$$
\left\|\mathbf{P}_{\leq N} \mathcal{N}(v+z)\right\|_{L_{t}^{1}\left([0, T) ; H_{x}^{\frac{d-2}{2}}\right)} \lesssim N^{\frac{d-2}{2}}\|\mathcal{N}(v+z)\|_{L_{t}^{1} L_{x}^{2}}
$$

On the one hand, it follows from Lemma 2.2 that the second term on the righthand side of (4.8) is finite almost surely. On the other hand, noting that $\left(3, \frac{6 d}{3 d-4}\right)$ is Strichartz admissible, it follows from Sobolev's inequality and (3.4) in Lemma 3.5 that

$$
\|v\|_{L_{t}^{3}\left([0, T) ; L_{x}^{6}\right)} \lesssim\left\|\langle\nabla\rangle^{\frac{d-2}{3}} v\right\|_{L_{t}^{3}\left([0, T) ; L_{x}^{\frac{6 d}{3 d-4}}\right)} \lesssim\|v\|_{X^{\frac{d-2}{3}}([0, T))}<\infty .
$$

Therefore, by Lemma 3.5 (i), we have

$$
\left\|\Gamma_{N} v(t)\right\|_{X^{\frac{d-2}{2}}} \lesssim \sup _{\substack{v_{4} \in Y^{0}([0, T)) \\\left\|v_{4}\right\|_{Y^{0}=1}}}\left|\int_{0}^{T} \int_{\mathbb{R}^{d}}\langle\nabla\rangle^{\frac{d-2}{2}} \mathcal{N}(v+z)(t, x) \overline{v_{4}(t, x)} d x d t\right|,
$$

almost surely, where $v_{4}=\mathbf{P}_{\leq N} v_{4}$. In the following, we estimate the right-hand side of (4.10), independently of the cutoff size $N \geq 1$, by performing a case-by-case analysis of expressions of the form:

$$
\left|\int_{0}^{T} \int_{\mathbb{R}^{d}}\langle\nabla\rangle^{\frac{d-2}{2}}\left(w_{1} w_{2} w_{3}\right) v_{4} d x d t\right|
$$

where $\left\|v_{4}\right\|_{Y^{0}([0, T))} \leq 1$ and $w_{j}=v$ or $z, j=1,2,3$. As a result, by taking $N \rightarrow \infty$, the same estimates hold for $\Gamma v$ without any cutoff, thus yielding (4.2).

Before proceeding further, let us simplify some of the notation. In the following, we drop the complex conjugate sign. We also denote $X^{s}([0, T))$ and $Y^{s}([0, T))$ by $X^{s}$ and $Y^{s}$ since $T$ is fixed. Similarly, it is understood that the time integration in $L_{t, x}^{p}$ is over $[0, T)$. Lastly, in most of the cases, we dyadically decompose $w_{j}=v_{j}$ or $z_{j}, j=1,2,3$, and $v_{4}$ such that their spatial frequency supports are $\left\{\left|\xi_{j}\right| \sim N_{j}\right\}$ for some dyadic $N_{j} \geq 1$ but still denote them as $w_{j}=v_{j}$ or $z_{j}, j=1,2,3$, and $v_{4}$. 
Note that, if we can afford a small derivative loss in the largest frequency, there is no difficulty in summing over the dyadic blocks $N_{j}, j=1, \ldots, 4$.

Case (1): vvv case. In this case, we do not need to perform dyadic decompositions and we divide the frequency spaces into $\left\{\left|\xi_{1}\right| \geq\left|\xi_{2}\right|,\left|\xi_{3}\right|\right\},\left\{\left|\xi_{2}\right| \geq\left|\xi_{1}\right|,\left|\xi_{3}\right|\right\}$, and $\left\{\left|\xi_{3}\right| \geq\left|\xi_{1}\right|,\left|\xi_{2}\right|\right\}$. Without loss of generality, assume that $\left|\xi_{1}\right| \geq\left|\xi_{2}\right|,\left|\xi_{3}\right|$. By the $L_{t, x}^{\frac{2(d+2)}{d}} L_{t, x}^{d+2} L_{t, x}^{d+2} L_{t, x}^{\frac{2(d+2)}{d}}$-Hölder's inequality, (3.5) in Lemma 3.5, and (3.2), we have

$$
\begin{aligned}
\left|\int_{0}^{T} \int_{\mathbb{R}^{d}}\langle\nabla\rangle^{\frac{d-2}{2}} v_{1} v_{2} v_{3} v_{4} d x d t\right| & \leq\left\|\langle\nabla\rangle^{\frac{d-2}{2}} v_{1}\right\|_{L_{t, x} \frac{2(d+2)}{d}}\left\|v_{2}\right\|_{L_{t, x}^{d+2}}\left\|v_{3}\right\|_{L_{t, x}^{d+2}}\left\|v_{4}\right\|_{L_{t, x} \frac{2(d+2)}{d}} \\
& \lesssim \prod_{j=1}^{3}\left\|v_{j}\right\|_{Y^{\frac{d-2}{2}}}\left\|v_{4}\right\|_{Y^{0}} \lesssim \prod_{j=1}^{3}\left\|v_{j}\right\|_{X^{\frac{d-2}{2}}}
\end{aligned}
$$

Case (2): zzz case. Without loss of generality, assume $N_{3} \geq N_{2} \geq N_{1}$.



$$
\begin{aligned}
\mid \int_{0}^{T} \int_{\mathbb{R}^{d}} & z_{1} z_{2}\langle\nabla\rangle^{\frac{d-2}{2}} z_{3} v_{4} d x d t \mid \\
& \left.\lesssim\left\|z_{1}\right\|_{L_{t, x}^{d+2}}\left\|\langle\nabla\rangle^{\frac{d-2}{4}} z_{2}\right\|_{L_{t, x}^{4}} \|\langle\nabla\rangle\right\rangle^{\frac{d-2}{4}} z_{3} \|_{L_{t, x}^{4}\left\|v_{4}\right\|_{L_{t, x}^{\frac{2(d+2)}{d}}}}
\end{aligned}
$$

Hence, by Lemmata 2.2 and 3.5, the contribution to (4.10) in this case is at most $\lesssim T^{0+} R^{3}$ outside a set of probability

$$
\leq C \exp \left(-c \frac{R^{2}}{T^{\frac{2}{d+2}}-\|\phi\|_{L^{2}}^{2}}\right)+C \exp \left(-c \frac{R^{2}}{T^{\frac{1}{2}-}\|\phi\|_{H^{\frac{d-2}{4}+}}^{2}}\right)
$$

as long as $s>\frac{d-2}{4}$. Note that $s$ needs to be strictly greater than $\frac{d-2}{4}$ due to the summations over dyadic blocks. See [4] for more details. Similar comments apply in the following.

- Subcase (2.b): $N_{3} \sim N_{4} \gg N_{1}, N_{2}$.

○ Subsubcase (2.b.i): $N_{1}, N_{2} \ll N_{3}^{\frac{1}{d-1}}$. For small $\alpha>0$, it follows from CauchySchwarz' inequality and Lemma 3.5 that

$$
\begin{aligned}
\left\|z_{2}\langle\nabla\rangle^{\frac{d-2}{2}} z_{3}\right\|_{L_{t, x}^{2}} & \lesssim N_{3}^{\frac{d-2}{2}}\left\|z_{2}\right\|_{L_{t, x}^{4}}^{\alpha}\left\|z_{3}\right\|_{L_{t, x}^{4}}^{\alpha}\left\|z_{2} z_{3}\right\|_{L_{t, x}^{2}}^{1-\alpha} \\
& \lesssim N_{2}^{\frac{d-1}{2}-s-\frac{d-1}{2} \alpha-} N_{3}^{\frac{d-3}{2}-s+\frac{1}{2} \alpha+} \prod_{j=2}^{3}\left(\left\|\langle\nabla\rangle^{s} z_{j}\right\|_{L_{t, x}^{4}}^{\alpha}\left\|\mathbf{P}_{N_{j}} \phi^{\omega}\right\|_{H^{s}}^{1-\alpha}\right) .
\end{aligned}
$$


Then, by (4.12) and the bilinear estimate (3.6) in Lemma 3.5] we have

$$
\begin{gathered}
\left|\int_{0}^{T} \int_{\mathbb{R}^{d}} z_{1} z_{2}\langle\nabla\rangle^{\frac{d-2}{2}} z_{3} v_{4} d x d t\right| \lesssim\left\|z_{2}\langle\nabla\rangle^{\frac{d-2}{2}} z_{3}\right\|_{L_{t, x}^{2}}\left\|z_{1} v_{4}\right\|_{L_{t, x}^{2}} \\
\lesssim N_{1}^{\frac{d-1}{2}-s-} N_{2}^{\frac{d-1}{2}-s-\frac{d-1}{2} \alpha-} N_{3}^{\frac{d-4}{2}-s+\frac{1}{2} \alpha+} \\
\quad \times\left\|\mathbf{P}_{N_{1}} \phi^{\omega}\right\|_{H^{s}} \prod_{j=2}^{3}\left(\left\|\langle\nabla\rangle^{s} z_{j}\right\|_{L_{t, x}^{4}}^{\alpha}\left\|\mathbf{P}_{N_{j}} \phi^{\omega}\right\|_{H^{s}}^{1-\alpha}\right)\left\|v_{4}\right\|_{Y^{0}} \\
\lesssim N_{3}^{\frac{d-2}{2}-\frac{d+1}{d-1} s+}\left\|\mathbf{P}_{N_{1}} \phi^{\omega}\right\|_{H^{s}} \prod_{j=2}^{3}\left(\left\|\langle\nabla\rangle^{s} z_{j}\right\|_{L_{t, x}^{4}}^{\alpha}\left\|\mathbf{P}_{N_{j}} \phi^{\omega}\right\|_{H^{s}}^{1-\alpha}\right)\left\|v_{4}\right\|_{Y^{0}} .
\end{gathered}
$$

Hence, by Lemmata 2.2 and 2.4, the contribution to (4.10) in this case is at most $\lesssim T^{0+} R^{3}$ outside a set of probability

$$
\leq C \exp \left(-c \frac{R^{2}}{T^{\frac{1}{2}-}\|\phi\|_{H^{s}}^{2}}\right)+C \exp \left(-c \frac{R^{2}}{\|\phi\|_{H^{s}}^{2}}\right)
$$

as long as

$$
s>\frac{d-1}{d+1} \cdot \frac{d-2}{2}=s_{d}
$$

and $\alpha<1-\frac{2}{d-1} s$.

○ Subsubcase (2.b.ii): $N_{2} \gtrsim N_{3}^{\frac{1}{d-1}} \gg N_{1}$. By Hölder's inequality and the bilinear estimate (3.6) in Lemma 3.5, we have

$$
\begin{aligned}
\left|\int_{0}^{T} \int_{\mathbb{R}^{d}} z_{1} z_{2}\langle\nabla\rangle^{\frac{d-2}{2}} z_{3} v_{4} d x d t\right| \lesssim\left\|z_{2}\right\|_{L_{t, x}^{4}}\left\|\langle\nabla\rangle^{\frac{d-2}{2}} z_{3}\right\|_{L_{t, x}^{4}}\left\|z_{1} v_{4}\right\|_{L_{t, x}^{2}} \\
\lesssim N_{1}^{\frac{d-1}{2}-s-} N_{2}^{-s} N_{3}^{\frac{d-3}{2}-s+}\left\|\mathbf{P}_{N_{1}} \phi^{\omega}\right\|_{H^{s}} \prod_{j=2}^{3}\left\|\langle\nabla\rangle^{s} z_{j}\right\|_{L_{t, x}^{4}}\left\|v_{4}\right\|_{Y^{0}} \\
\lesssim N_{3}^{\frac{d-2}{2}-\frac{d+1}{d-1} s+}\left\|\mathbf{P}_{N_{1}} \phi^{\omega}\right\|_{H^{s}} \prod_{j=2}^{3}\left\|\langle\nabla\rangle^{s} z_{j}\right\|_{L_{t, x}^{4}}\left\|v_{4}\right\|_{Y^{0}} .
\end{aligned}
$$

Hence, by Lemmata 2.2 and 2.4, the contribution to (4.10) in this case is at most $\lesssim T^{0+} R^{3}$ outside a set of probability

$$
\leq C \exp \left(-c \frac{R^{2}}{T^{\frac{1}{2}-}\|\phi\|_{H^{s}}^{2}}\right)+C \exp \left(-c \frac{R^{2}}{\|\phi\|_{H^{s}}^{2}}\right)
$$

as long as (4.13) is satisfied.

○ Subsubcase (2.b.iii): $N_{1}, N_{2} \gtrsim N_{3}^{\frac{1}{d-1}}$. By the $L_{t, x}^{\frac{6(d+2)}{d+4}} L_{t, x}^{\frac{6(d+2)}{d+4}} L_{t, x}^{\frac{6(d+2)}{d+4}} L_{t, x}^{\frac{2(d+2)}{d}}$-Hölder's inequality and (3.5) in Lemma 3.5, we have

$$
\left|\int_{0}^{T} \int_{\mathbb{R}^{d}} z_{1} z_{2}\langle\nabla\rangle^{\frac{d-2}{2}} z_{3} v_{4} d x d t\right| \lesssim N_{3}^{\frac{d-2}{2}-\frac{d+1}{d-1} s} \prod_{j=1}^{3}\left\|\langle\nabla\rangle^{s} z_{j}\right\|_{L_{t, x}^{\frac{6(d+2)}{d+4}}}\left\|v_{4}\right\|_{Y^{0}} .
$$


Hence, by Lemma 2.2, the contribution to (4.10) in this case is at most $\lesssim T^{0+} R^{3}$ outside a set of probability

$$
\leq C \exp \left(-c \frac{R^{2}}{T^{\frac{d+4}{3(d+2)}-}\|\phi\|_{H^{s}}^{2}}\right)
$$

as long as (4.13) is satisfied.

Case (3): vvz case. Without loss of generality, assume $N_{1} \geq N_{2}$.

- Subcase (3.a): $N_{1} \gtrsim N_{3}$. In the following, we apply dyadic decompositions only to $v_{1}, v_{2}$, and $z_{3}$. In this case, we have $N_{1} \sim \max \left(N_{2}, N_{3},\left|\xi_{4}\right|\right)$, where $\xi_{4}$ is the spatial frequency of $v_{4}$. Then, by Hölder's inequality, (3.6), and (3.5), we have

$$
\begin{aligned}
&\left|\int_{0}^{T} \int_{\mathbb{R}^{d}}\langle\nabla\rangle^{\frac{d-2}{2}} v_{1} v_{2} z_{3} v_{4} d x d t\right| \\
& \lesssim \sum_{N_{1} \gtrsim N_{2}, N_{3}}\left\|\langle\nabla\rangle^{\frac{d-2}{2}} \mathbf{P}_{N_{1}} v_{1} \mathbf{P}_{N_{2}} v_{2}\right\|_{L_{t, x}^{2}}\left\|\mathbf{P}_{N_{3}} z_{3}\right\|_{L_{t, x}^{d+2}}\left\|v_{4}\right\|_{L_{t, x}^{\frac{2(d+2)}{d}}} \\
& \lesssim \sum_{N_{1} \geq N_{2}}\left(\frac{N_{2}}{N_{1}}\right)^{\frac{1}{2}-} \prod_{j=1}^{2}\left\|\mathbf{P}_{N_{j}} v_{j}\right\|_{X^{\frac{d-2}{2}}} \sum_{N_{3}}\left\|\mathbf{P}_{N_{3}} z_{3}\right\|_{L_{t, x}^{d+2}}\left\|v_{4}\right\|_{Y^{0}}
\end{aligned}
$$

By Lemma 3.6 and summing over $N_{3}$ with a slight loss of derivative,

$$
\lesssim \prod_{j=1}^{2}\left\|v_{j}\right\|_{X^{\frac{d-2}{2}}}\left\|\langle\nabla\rangle^{0+} z_{3}\right\|_{L_{t, x}^{d+2}}\left\|v_{4}\right\|_{Y^{0}} .
$$

Hence, by Lemma 2.2. the contribution to (4.10) in this case is at most $\lesssim T^{0+} R \prod_{j=1}^{2}\left\|v_{j}\right\|_{X^{\frac{d-2}{2}}}$ outside a set of probability

$$
\leq C \exp \left(-c \frac{R^{2}}{T^{\frac{2}{d+2}}-\|\phi\|_{H^{0+}}^{2}}\right)
$$

as long as $s>0$.

- Subcase (3.b): $N_{3} \sim N_{4} \gg N_{1} \geq N_{2}$.

○ Subsubcase (3.b.i): $N_{1} \gtrsim N_{3}^{\frac{1}{d-1}}$. By Hölder's inequality followed by (3.5) and (3.6) in Lemma 3.5, we have

$$
\begin{aligned}
& \left|\int_{0}^{T} \int_{\mathbb{R}^{d}} v_{1} v_{2}\langle\nabla\rangle^{\frac{d-2}{2}} z_{3} v_{4} d x d t\right| \lesssim\left\|v_{1}\right\|_{L_{t, x}^{\frac{2(d+2)}{d}}}\left\|\langle\nabla\rangle^{\frac{d-2}{2}} z_{3}\right\|_{L_{t, x}^{d+2}}\left\|v_{2} v_{4}\right\|_{L_{t, x}^{2}} \\
& \lesssim N_{1}^{-\frac{d-2}{2}} N_{2}^{\frac{1}{2}-} N_{3}^{\frac{d-3}{2}-s+}\left\|v_{1}\right\|_{X \frac{d-2}{2}}\left\|v_{2}\right\|_{X} \frac{d-2}{2}\left\|\langle\nabla\rangle^{s} z_{3}\right\|_{L_{t, x}^{d+2}}\left\|v_{4}\right\|_{Y^{0}} \\
& \lesssim N_{3}^{\frac{d-3}{d-1} \frac{d-2}{2}-s+}\left\|v_{1}\right\|_{X \frac{d-2}{2}}\left\|v_{2}\right\|_{X \frac{d-2}{2}}\left\|\langle\nabla\rangle^{s} z_{3}\right\|_{L_{t, x}^{d+2}}\left\|v_{4}\right\|_{Y^{0}} .
\end{aligned}
$$

Hence, by Lemma 2.2, the contribution to (4.10) in this case is at most $\lesssim T^{0+} R \prod_{j=1}^{2}\left\|v_{j}\right\|_{X \frac{d-2}{2}}$ outside a set of probability

$$
\leq C \exp \left(-c \frac{R^{2}}{T^{\frac{2}{d+2}}-\|\phi\|_{H^{s}}^{2}}\right)
$$


as long as

$$
s>\frac{d-3}{d-1} \cdot \frac{d-2}{2}
$$

Note that the condition (4.14) is less restrictive than (4.13).

○ Subsubcase (3.b.ii): $N_{2} \leq N_{1} \ll N_{3}^{\frac{1}{d-1}}$. For small $\alpha>0$, it follows from Hölder's inequality and Lemma 3.5 that

$$
\begin{aligned}
\left\|v_{1}\langle\nabla\rangle^{\frac{d-2}{2}} z_{3}\right\|_{L_{t, x}^{2}} & \lesssim N_{3}^{\frac{d-2}{2}}\left\|v_{1}\right\|_{L_{t, x}^{\left(\frac{2(d+2)}{d}\right.}}^{\alpha}\left\|z_{3}\right\|_{L_{t, x}^{d+2}}^{\alpha}\left\|v_{1} z_{3}\right\|_{L_{t, x}^{2}}^{1-\alpha} \\
& \lesssim N_{1}^{\frac{1-\alpha}{2}-\frac{d-1}{2} \alpha-} N_{3}^{\frac{d-3}{2}-s+\frac{1}{2} \alpha+}\left\|v_{1}\right\|_{X \frac{d-2}{2}}\left\|\langle\nabla\rangle^{s} z_{3}\right\|_{L_{t, x}^{d+2}}^{\alpha}\left\|\mathbf{P}_{N_{3}} \phi^{\omega}\right\|_{H^{s}}^{1-\alpha} .
\end{aligned}
$$

Then, by (4.15) and (3.6) in Lemma 3.5 we have

$$
\begin{gathered}
\left|\int_{0}^{T} \int_{\mathbb{R}^{d}} v_{1} v_{2}\langle\nabla\rangle^{\frac{d-2}{2}} z_{3} v_{4} d x d t\right| \lesssim\left\|v_{1}\langle\nabla\rangle^{\frac{d-2}{2}} z_{3}\right\|_{L_{t, x}^{2}}\left\|v_{2} v_{4}\right\|_{L_{t, x}^{2}} \\
\lesssim N_{1}^{\frac{1}{2}-\frac{d-1}{2} \alpha-} N_{2}^{\frac{1}{2}-} N_{3}^{\frac{d-4}{2}-s+\frac{1}{2} \alpha+}\left\|v_{1}\right\|_{X^{\frac{d-2}{2}}}\left\|v_{2}\right\|_{X^{\frac{d-2}{2}}} \\
\times\left\|\langle\nabla\rangle^{s} z_{3}\right\|_{L_{t, x}^{d+2}}^{\alpha}\left\|\mathbf{P}_{N_{3}} \phi^{\omega}\right\|_{H^{s}}^{1-\alpha}\left\|v_{4}\right\|_{Y^{0}} .
\end{gathered}
$$

Hence, by Lemmata 2.2 and 2.4 , the contribution to (4.10) in this case is at most $\lesssim T^{0+} R \prod_{j=1}^{2}\left\|v_{j}\right\|_{X \frac{d-2}{2}}$ outside a set of probability

$$
\leq C \exp \left(-c \frac{R^{2}}{T^{\frac{2}{d+2}}-\|\phi\|_{H^{s}}^{2}}\right)+C \exp \left(-c \frac{R^{2}}{\|\phi\|_{H^{s}}^{2}}\right)
$$

as long as (4.14) is satisfied and $\alpha<\frac{1}{d-1}$.

Case (4): vzz case. Without loss of generality, assume $N_{3} \geq N_{2}$.

- Subcase (4.a): $N_{1} \gtrsim N_{3}$. By the $L_{t, x}^{\frac{2(d+2)}{d}} L_{t, x}^{d+2} L_{t, x}^{d+2} L_{t, x}^{\frac{2(d+2)}{d}}$-Hölder's inequality and (3.5) in Lemma 3.5. we have

$$
\left|\int_{0}^{T} \int_{\mathbb{R}^{d}}\langle\nabla\rangle^{\frac{d-2}{2}} v_{1} z_{2} z_{3} v_{4} d x d t\right| \lesssim\left\|v_{1}\right\|_{X^{\frac{d-2}{2}}}\left\|z_{2}\right\|_{L_{t, x}^{d+2}}\left\|z_{3}\right\|_{L_{t, x}^{d+2}}\left\|v_{4}\right\|_{Y^{0}} .
$$

Hence, by Lemma 2.2. the contribution to 4.10 in this case is at most $\lesssim T^{0+} R^{2}\left\|v_{1}\right\|_{X \frac{d-2}{2}}$ outside a set of probability

$$
\leq C \exp \left(-c \frac{R^{2}}{T^{\frac{2}{d+2}}-\|\phi\|_{H^{0+}}^{2}}\right)
$$

as long as $s>0$. As before, we have $\|\phi\|_{H^{0+}}$ instead of $\|\phi\|_{L^{2}}$ in (4.16), allowing us to sum over $N_{2}$ and $N_{3}$. If $N_{3} \gtrsim \max \left(N_{1}, N_{4}\right)$, then this also allows us to sum over $N_{1}$ and $N_{4}$. Otherwise, we have $N_{1} \sim N_{4} \gg N_{3}$. In this case, we can use Cauchy-Schwarz' inequality to sum over $N_{1} \sim N_{4}$. 
- Subcase (4.b): $N_{3} \gg N_{1}$. First, suppose that $N_{2} \sim N_{3}$. Note that we must have $N_{3} \gtrsim N_{4}$ in this case. Then, by the $L_{t, x}^{d+2} L_{t, x}^{4} L_{t, x}^{4} L_{t, x}^{\frac{2(d+2)}{d}}$-Hölder's inequality with (3.5) in Lemma 3.5, we have

$$
\begin{aligned}
\mid \int_{0}^{T} \int_{\mathbb{R}^{d}} & v_{1} z_{2}\langle\nabla\rangle^{\frac{d-2}{2}} z_{3} v_{4} d x d t \mid \\
& \lesssim\left\|v_{1}\right\|_{X^{\frac{d-2}{2}}}\left\|\langle\nabla\rangle^{\frac{d-2}{4}} z_{2}\right\|_{L_{t, x}^{4}}\left\|\langle\nabla\rangle^{\frac{d-2}{4}} z_{3}\right\|_{L_{t, x}^{4}}\left\|v_{4}\right\|_{Y^{0}} \\
& \lesssim N_{3}^{\frac{d-2}{2}-2 s}\left\|v_{1}\right\|_{X^{\frac{d-2}{2}}}\left\|\langle\nabla\rangle^{s} z_{2}\right\|_{L_{t, x}^{4}}\left\|\langle\nabla\rangle^{s} z_{3}\right\|_{L_{t, x}^{4}}\left\|v_{4}\right\|_{Y^{0}}
\end{aligned}
$$

Hence, by Lemma 2.2. the contribution to (4.10) in this case is at most $\lesssim T^{0+} R^{2}\left\|v_{1}\right\|_{X^{\frac{d-2}{2}}}$ outside a set of probability

$$
\leq C \exp \left(-c \frac{R^{2}}{T^{\frac{1}{2}-}\|\phi\|_{H^{s}}^{2}}\right)
$$

as long as $s>\frac{d-2}{4}$.

Hence, it remains to consider the case $N_{3} \sim N_{4} \gg N_{1}, N_{2}$.

○ Subsubcase (4.b.i): $N_{1}, N_{2} \ll N_{3}^{\frac{1}{d-1}}$. By (4.12) and (3.6) in Lemma 3.5, we have

$$
\begin{gathered}
\left|\int_{0}^{T} \int_{\mathbb{R}^{d}} v_{1} z_{2}\langle\nabla\rangle^{\frac{d-2}{2}} z_{3} v_{4} d x d t\right| \lesssim\left\|z_{2}\langle\nabla\rangle^{\frac{d-2}{2}} z_{3}\right\|_{L_{t, x}^{2}}\left\|v_{1} v_{4}\right\|_{L_{t, x}^{2}} \\
\lesssim N_{1}^{\frac{1}{2}-} N_{2}^{\frac{d-1}{2}-s-\frac{d-1}{2} \alpha-} N_{3}^{\frac{d-4}{2}-s+\frac{1}{2} \alpha+}\left\|v_{1}\right\|_{X^{\frac{d-2}{2}}} \\
\times \prod_{j=2}^{3}\left(\left\|\langle\nabla\rangle^{s} z_{j}\right\|_{L_{t, x}^{4}}^{\alpha}\left\|\mathbf{P}_{N_{j}} \phi^{\omega}\right\|_{H^{s}}^{1-\alpha}\right)\left\|v_{4}\right\|_{Y^{0}} .
\end{gathered}
$$

Hence, by Lemmata 2.2 and 2.4, the contribution to (4.10) in this case is at most $\lesssim T^{0+} R^{2}\left\|v_{1}\right\|_{X \frac{d-2}{2}}$ outside a set of probability

$$
\leq C \exp \left(-c \frac{R^{2}}{T^{\frac{1}{2}-}\|\phi\|_{H^{s}}^{2}}\right)+C \exp \left(-c \frac{R^{2}}{\|\phi\|_{H^{s}}^{2}}\right)
$$

as long as

$$
s>\frac{(d-2)^{2}}{2 d}=\frac{d-2}{d} \cdot \frac{d-2}{2}
$$

and $\alpha<1-\frac{2}{d-1} s$. Note that the condition (4.17) is less restrictive than (4.13) and thus does not add a further constraint.

○ Subsubcase (4.b.ii): $N_{1} \ll N_{3}^{\frac{1}{d-1}} \lesssim N_{2}$. By Hölder's inequality and (3.6) in Lemma 3.5. we have

$$
\begin{aligned}
\left|\int_{0}^{T} \int_{\mathbb{R}^{d}} v_{1} z_{2}\langle\nabla\rangle^{\frac{d-2}{2}} z_{3} v_{4} d x d t\right| \lesssim\left\|z_{2}\right\|_{L_{t, x}^{4}}\left\|\langle\nabla\rangle^{\frac{d-2}{2}} z_{3}\right\|_{L_{t, x}^{4}}\left\|v_{1} v_{4}\right\|_{L_{t, x}^{2}} \\
\lesssim N_{1}^{\frac{1}{2}-} N_{2}^{-s} N_{3}^{\frac{d-3}{2}-s+}\left\|v_{1}\right\|_{X} \frac{d-2}{2} \prod_{j=2}^{3}\left\|\langle\nabla\rangle^{s} z_{j}\right\|_{L_{t, x}^{4}}\left\|v_{4}\right\|_{Y^{0}}
\end{aligned}
$$


Hence, by Lemma 2.2, the contribution to (4.10) in this case is at most $\lesssim T^{0+} R^{2}\left\|v_{1}\right\|_{X^{\frac{d-2}{2}}}$ outside a set of probability

$$
\leq C \exp \left(-c \frac{R^{2}}{T^{\frac{1}{2}-}\|\phi\|_{H^{s}}^{2}}\right)
$$

as long as (4.17) is satisfied.

○ Subsubcase (4.b.iii): $N_{2} \ll N_{3}^{\frac{1}{d-1}} \lesssim N_{1}$. By Hölder's inequality and Lemma 3.5 . we have

$$
\begin{aligned}
\left|\int_{0}^{T} \int_{\mathbb{R}^{d}} v_{1} z_{2}\langle\nabla\rangle^{\frac{d-2}{2}} z_{3} v_{4} d x d t\right| \lesssim\left\|v_{1}\right\|_{L_{t, x} \frac{2(d+2)}{d}}\left\|\langle\nabla\rangle^{\frac{d-2}{2}} z_{3}\right\|_{L_{t, x}^{d+2}}\left\|z_{2} v_{4}\right\|_{L_{t, x}^{2}} \\
\\
\lesssim N_{1}^{-\frac{d-2}{2}} N_{2}^{\frac{d-1}{2}-s-} N_{3}^{\frac{d-3}{2}-s+}\left\|v_{1}\right\|_{X^{\frac{d-2}{2}}}\left\|\mathbf{P}_{N_{2}} \phi^{\omega}\right\|_{H^{s}}\left\|\langle\nabla\rangle^{s} z_{3}\right\|_{L_{t, x}^{d+2}}\left\|v_{4}\right\|_{Y^{0}} .
\end{aligned}
$$

Hence, by Lemmata 2.4 and 2.2, the contribution to (4.10) in this case is at most $\lesssim T^{0+} R^{2}\left\|v_{1}\right\|_{X \frac{d-2}{2}}$ outside a set of probability

$$
\leq C \exp \left(-c \frac{R^{2}}{\|\phi\|_{H^{s}}^{2}}\right)+C \exp \left(-c \frac{R^{2}}{T^{\frac{2}{d+2}}-\|\phi\|_{H^{s}}^{2}}\right)
$$

as long as (4.17) is satisfied.

○ Subsubcase (4.b.iv): $N_{1}, N_{2} \gtrsim N_{3}^{\frac{1}{d-1}}$. By the $L_{t, x}^{\frac{2(d+2)}{d}} L_{t, x}^{d+2} L_{t, x}^{d+2} L_{t, x}^{\frac{2(d+2)}{d}}$-Hölder's inequality and (3.5) in Lemma 3.5. we have

$$
\begin{aligned}
\mid \int_{0}^{T} \int_{\mathbb{R}^{d}} & v_{1} z_{2}\langle\nabla\rangle^{\frac{d-2}{2}} z_{3} v_{4} d x d t \mid \\
& \lesssim\left\|v_{1}\right\|_{L_{t, x}^{\frac{2(d+2)}{d}}\left\|z_{2}\right\|_{L_{t, x}^{d+2}}\left\|\langle\nabla\rangle^{\frac{d-2}{2}} z_{3}\right\|_{L_{t, x}^{d+2}}\left\|v_{4}\right\|_{L_{t, x} \frac{2(d+2)}{d}}} \\
& \lesssim N_{1}^{-\frac{d-2}{2}} N_{2}^{-s} N_{3}^{\frac{d-2}{2}-s}\left\|v_{1}\right\|_{X} \frac{d-2}{2} \prod_{j=2}^{3}\left\|\langle\nabla\rangle^{s} z_{j}\right\|_{L_{t, x}^{d+2}}\left\|v_{4}\right\|_{Y^{0}} .
\end{aligned}
$$

Hence, by Lemma 2.2, the contribution to (4.10 in this case is at most $\lesssim T^{0+} R^{2}\left\|v_{1}\right\|_{X^{\frac{d-2}{2}}}$ outside a set of probability

$$
\leq C \exp \left(-c \frac{R^{2}}{T^{\frac{2}{d+2}}-\|\phi\|_{H^{s}}^{2}}\right)
$$

as long as (4.17) is satisfied.

Putting together Cases (1) - (4) above, the conclusion of part (i) follows, provided that (4.13) is satisfied.

(ii) First, define $\widetilde{\Gamma}_{N}$ by

$$
\widetilde{\Gamma}_{N} v(t)=\mp i \int_{0}^{t} S\left(t-t^{\prime}\right) \mathbf{P}_{\leq N} \mathcal{N}(v+\varepsilon z)\left(t^{\prime}\right) d t^{\prime}
$$

for $N \geq 1$. As before, we have

$$
\left\|\mathbf{P}_{\leq N} \mathcal{N}(v+\varepsilon z)\right\|_{L_{t}^{1}\left(\mathbb{R} ; H_{x}^{\frac{d-2}{2}}\right)} \lesssim N^{\frac{d-2}{2}}\|v\|_{L_{t}^{3}\left(\mathbb{R} ; L_{x}^{6}\right)}^{3}+\varepsilon^{3} N^{\frac{d-2}{2}}\|z\|_{L_{t}^{3}\left(\mathbb{R} ; L_{x}^{6}\right)}^{3} .
$$

By a computation similar to (4.9), we see that the first term is finite. Noting that $\left(3, \frac{6 d}{3 d-4}\right)$ is Strichartz admissible and $6 \geq \frac{6 d}{3 d-4}$, it follows from Lemma 2.3 that the 
second term on the right-hand side of (4.18) is finite almost surely. Hence, we can apply Lemma 3.5 (i) to $\widetilde{\Gamma}_{N} v$ for each finite $N \geq 1$, almost surely.

The rest of the proof for this part follows in a similar manner to the proof of part (i) by changing the time interval from $[0, T)$ to $\mathbb{R}$ and replacing $z$ by $\varepsilon z$. By applying Lemma 2.3 instead of Lemma 2.2 in the above computation, we see that the contribution to (4.10), where $[0, T)$ is replaced by $\mathbb{R}$, is given by

$$
\text { Case (2): } R^{3}, \quad \text { Case (3): } R \prod_{j=1}^{2}\left\|v_{j}\right\|_{X \frac{d-2}{2}(\mathbb{R})}, \quad \text { Case (4): } R^{2}\left\|v_{1}\right\|_{X^{\frac{d-2}{2}}(\mathbb{R})}
$$

outside a set of probability

$$
\leq C \exp \left(-c \frac{R^{2}}{\varepsilon^{2}\|\phi\|_{H^{s}}^{2}}\right)
$$

in all cases as long as $s>s_{d}$.

\section{Proofs of Theorems 1.1 and 1.2}

In this section, we establish the almost sure local well-posedness (Theorem 1.1) and probabilistic small data global theory (Theorem 1.2). First, we present the proof of Theorem 1.1. Given $C_{1}$ and $C_{2}$ as in (4.2) and (4.3), let $\eta_{1}>0$ be sufficiently small such that

$$
C_{1} \eta_{1}^{2} \leq \frac{1}{2} \quad \text { and } \quad 2 C_{2} \eta_{1}^{2} \leq \frac{1}{4} .
$$

Also, given $R \gg 1$, choose $T=T(R)$ such that

$$
T^{\theta}=\min \left(\frac{\eta_{1}}{2 C_{1} R^{3}}, \frac{1}{4 C_{2} R^{2}}\right) .
$$

Then, it follows from Proposition 4.1 that $\Gamma$ is a contraction on the ball $B_{\eta_{1}}$ defined by

$$
B_{\eta_{1}}:=\left\{u \in X^{\frac{d-2}{2}}([0, T)) \cap C\left([0, T) ; H^{\frac{d-2}{2}}\right):\|u\|_{X^{\frac{d-2}{2}}([0, T))} \leq \eta_{1}\right\}
$$

outside a set of probability

$$
\leq C \exp \left(-c \frac{R^{2}}{\|\phi\|_{H^{s}}^{2}}\right) \sim C \exp \left(-c \frac{1}{T^{\gamma}\|\phi\|_{H^{s}}^{2}}\right)
$$

for some $\gamma>0$. This proves Theorem 1.1 .

Next, we prove Theorem 1.2. Let $\eta_{2}>0$ be sufficiently small such that

$$
2 C_{3} \eta_{2}^{2} \leq 1 \quad \text { and } \quad 3 C_{4} \eta_{2}^{2} \leq \frac{1}{2}
$$

where $C_{3}$ and $C_{4}$ are as in (4.5) and (4.6). Then, by Proposition 4.1 with $R=\eta_{2}$ and $\phi^{\omega}$ replaced by $\varepsilon \phi^{\omega}$, we have

$$
\begin{gathered}
\|\widetilde{\Gamma} v\|_{X^{\frac{d-2}{2}}(\mathbb{R})} \leq 2 C_{3} \eta_{2}^{3} \leq \eta_{2}, \\
\left\|\widetilde{\Gamma} v_{1}-\widetilde{\Gamma} v_{2}\right\|_{X^{\frac{d-2}{2}}(\mathbb{R})} \leq 3 C_{4} \eta_{2}^{2}\left\|v_{1}-v_{2}\right\|_{X^{\frac{d-2}{2}}(\mathbb{R})} \leq \frac{1}{2}\left\|v_{1}-v_{2}\right\|_{X^{\frac{d-2}{2}}(\mathbb{R})}
\end{gathered}
$$


outside a set of probability $\leq C \exp \left(-c \frac{\eta_{2}^{2}}{\varepsilon^{2}\|\phi\|_{H^{s}}^{2}}\right)$. Noting that $\eta_{2}$ is an absolute constant, we conclude that there exists a set $\Omega_{\varepsilon} \subset \Omega$ such that (i) $\widetilde{\Gamma}=\widetilde{\Gamma}^{\omega}$ is a contraction on the ball $B_{\eta_{2}}$ defined by

$$
B_{\eta_{2}}:=\left\{u \in X^{\frac{d-2}{2}}(\mathbb{R}) \cap C\left(\mathbb{R} ; H^{\frac{d-2}{2}}\right):\|u\|_{X^{\frac{d-2}{2}}(\mathbb{R})} \leq \eta_{2}\right\}
$$

for $\omega \in \Omega_{\varepsilon}$, and (ii) $P\left(\Omega_{\varepsilon}^{c}\right) \leq C \exp \left(-\frac{c}{\varepsilon^{2}\|\phi\|_{H^{s}}^{2}}\right)$. This proves global existence for (1.1) with initial data $\varepsilon \phi^{\omega}$ if $\omega \in \Omega_{\varepsilon}$.

Fix $\omega \in \Omega_{\varepsilon}$ and let $v=v(\varepsilon, \omega)$ be the global-in-time solution with $\left.v\right|_{t=0}=\varepsilon \phi^{\omega}$ constructed above. In order to prove scattering, we need to show that there exists $v_{+}^{\omega} \in H^{\frac{d-2}{2}}\left(\mathbb{R}^{d}\right)$ such that

$$
S(-t) v(t)=\mp i \int_{0}^{t} S\left(-t^{\prime}\right) \mathcal{N}(v+\varepsilon z)\left(t^{\prime}\right) d t^{\prime} \rightarrow v_{+}^{\omega}
$$

in $H^{\frac{d-2}{2}}\left(\mathbb{R}^{d}\right)$ as $t \rightarrow \infty$. With $w(t)=S(-t) v(t)$, define $I\left(t_{1}, t_{2}\right)$ and $\widetilde{I}\left(t_{1}, t_{2}\right)$ by

$$
\begin{aligned}
& I\left(t_{1}, t_{2}\right):=S\left(t_{2}\right)\left(w\left(t_{2}\right)-w\left(t_{1}\right)\right), \\
& \widetilde{I}\left(t_{1}, t_{2}\right):=\mp i \int_{0}^{t_{2}} S\left(t_{2}-t^{\prime}\right) \chi_{\left[t_{1}, \infty\right)}\left(t^{\prime}\right) \mathcal{N}(v+\varepsilon z)\left(t^{\prime}\right) d t^{\prime} .
\end{aligned}
$$

Then, for $0<t_{1} \leq t_{2}<\infty$, we have

$$
I\left(t_{1}, t_{2}\right)=\mp i S\left(t_{2}\right) \int_{t_{1}}^{t_{2}} S\left(-t^{\prime}\right) \mathcal{N}(v+\varepsilon z)\left(t^{\prime}\right) d t^{\prime}=\widetilde{I}\left(t_{1}, t_{2}\right) .
$$

Also, note that $\widetilde{I}\left(t_{1}, t_{2}\right)=0$ if $t_{1}>t_{2}$. In the following, we view $\widetilde{I}\left(t_{1}, t_{2}\right)$ as a function of $t_{2}$ and estimate its $X^{\frac{d-2}{2}}([0, \infty))$-norm. We now revisit the computation in the proof of Proposition 4.1 for $\widetilde{I}\left(t_{1}, t_{2}\right)$. In Case $(1)$, we proceed slightly differently. By Lemma 3.5 (i), Hölder's inequality, and (3.5), we have

$$
\begin{aligned}
\left\|\widetilde{I}\left(t_{1}, \cdot\right)\right\|_{X^{\frac{d-2}{2}}\left(\mathbb{R}_{+}\right)} & \lesssim \sup _{\substack{v_{4} \in Y^{0}\left(\mathbb{R}_{+}\right) \\
\left\|v_{4}\right\|_{Y^{0}=1}}}\left|\int_{0}^{\infty} \int_{\mathbb{R}^{d}} \chi_{\left[t_{1}, \infty\right)}(t)\langle\nabla\rangle^{\frac{d-2}{2}} v \bar{v} v v_{4} d x d t\right| \\
& \leq\left\|\langle\nabla\rangle^{\frac{d-2}{2}} v\right\|_{L_{t, x}^{\frac{2(d+2)}{d}}\left(\left[t_{1}, \infty\right)\right)}\|v\|_{L_{t, x}^{d+2}\left(\left[t_{1}, \infty\right)\right)}^{2} .
\end{aligned}
$$

By 3.5) in Lemma 3.5, we have

$$
\left\|\langle\nabla\rangle^{\frac{d-2}{2}} v\right\|_{L_{t, x}^{\frac{2(d+2)}{d}}(\mathbb{R})}+\|v\|_{L_{t, x}^{d+2}(\mathbb{R})} \lesssim\|v\|_{X}^{\frac{d-2}{2}(\mathbb{R})} \leq \eta_{2}
$$

Then, by the monotone convergence theorem, (5.6) tends to 0 as $t_{1} \rightarrow \infty$.

In Cases (2), (3), and (4), we had at least one factor of $z$. We multiply the cutoff function $\chi_{\left[t_{1}, \infty\right)}$ only on the $(\varepsilon z)$-factors but not on the $v$-factors. Note that $\|v\|_{X^{\frac{d-2}{2}}(\mathbb{R})} \leq \eta_{2}$. As in the proof of Proposition 4.1, we estimate at least a small portion of these $z$-factors in $\left\|\langle\nabla\rangle^{s} \varepsilon z^{\omega}\right\|_{L_{t, x}^{q}\left(\left[t_{1}, \infty\right)\right)}, q=4, \frac{6(d+2)}{d+4}$, or $d+2$, in each case. Recall that we have $\left\|\langle\nabla\rangle^{s} \varepsilon z^{\omega}\right\|_{L_{t, x}^{q}(\mathbb{R})} \leq \eta_{2}$ for $\omega \in \Omega_{\varepsilon}$. See Lemma2.3. Hence, again by the monotone convergence theorem, we have $\left\|\langle\nabla\rangle^{s} \varepsilon z^{\omega}\right\|_{L_{t, x}^{q}\left(\left[t_{1}, \infty\right)\right)} \rightarrow 0$ as $t_{1} \rightarrow \infty$ and thus the contribution from Cases (2), (3), and (4) tends to 0 as $t_{1} \rightarrow \infty$. Therefore, we have

$$
\lim _{t_{1} \rightarrow \infty}\left\|\widetilde{I}\left(t_{1}, t_{2}\right)\right\|_{X \frac{d-2}{2}}([0, \infty))=0 .
$$


In conclusion, we obtain

$$
\begin{aligned}
\lim _{t_{1} \rightarrow \infty} & \sup _{t_{2}>t_{1}}\left\|w\left(t_{2}\right)-w\left(t_{1}\right)\right\|_{H^{\frac{d-2}{2}}}=\lim _{t_{1} \rightarrow \infty} \sup _{t_{2}>t_{1}}\left\|I\left(t_{1}, t_{2}\right)\right\|_{H^{\frac{d-2}{2}}} \\
& =\lim _{t_{1} \rightarrow \infty}\left\|\widetilde{I}\left(t_{1}, t_{2}\right)\right\|_{L_{t_{2}}^{\infty}\left([0, \infty) ; H^{\frac{d-2}{2}}\right)} \lesssim \lim _{t_{1} \rightarrow \infty}\left\|\widetilde{I}\left(t_{1}, t_{2}\right)\right\|_{X^{\frac{d-2}{2}}([0, \infty))}=0 .
\end{aligned}
$$

This proves (5.5) and scattering of $u^{\omega}(t)=\varepsilon S(t) \phi^{\omega}+v^{\omega}(t)$, which completes the proof of Theorem 1.2 .

\section{LOCAL WELL-POSEDNESS OF NLS With A DETERMINISTIC PERTURBATION}

In this and the next sections, we consider the following Cauchy problem of the defocusing NLS with a perturbation:

$$
\left\{\begin{array}{l}
i \partial_{t} v+\Delta v=|v+f|^{2}(v+f) \\
\left.v\right|_{t=t_{0}}=v_{0}
\end{array}\right.
$$

where $f$ is a given deterministic function. Assuming some suitable conditions on $f$, we prove local well-posedness of (6.1) in this section (Proposition 6.3) and long time existence under further assumptions in Section 7 (Proposition 7.2). Then, we show, in Section 8 , that the conditions imposed on $f$ for long time existence are satisfied with a large probability by setting $f(t)=z(t)=S(t) \phi^{\omega}$. This yields Theorem 1.3 .

Our main goal is to prove long time existence of solutions to the perturbed NLS (6.1) by iteratively applying a perturbation lemma (Lemma 7.1). For this purpose, we first prove a "variant" local well-posedness of (6.1). As in the usual critical regularity theory, we first introduce an auxiliary scaling-invariant norm which is weaker than the $X^{\frac{d-2}{2}}$-norm. Given an interval $I \subset \mathbb{R}$, we introduce the $Z$-norm by

$$
\|u\|_{Z(I)}:=\left(\sum_{\substack{N \geq 1 \\ \text { dyadic }}} N^{d-2}\left\|\mathbf{P}_{N} u\right\|_{L_{t, x}^{4}\left(I \times \mathbb{R}^{d}\right)}^{4}\right)^{\frac{1}{4}} .
$$

By the Littlewood-Paley theory and (3.5) in Lemma 3.5 we have

$$
\|u\|_{Z(I)} \lesssim\left\|\langle\nabla\rangle^{\frac{d-2}{4}} u\right\|_{L_{t, x}^{4}\left(I \times \mathbb{R}^{d}\right)} \lesssim\|u\|_{X \frac{d-2}{2}(I)} .
$$

Given $\theta \in(0,1)$, we define the $Z_{\theta}$-norm by

$$
\|u\|_{Z_{\theta}(I)}:=\|u\|_{Z(I)}^{\theta}\|u\|_{X \frac{d-2}{2}(I)}^{1-\theta} .
$$

Note that the $Z_{\theta}$-norm is weaker than the $X^{\frac{d-2}{2}}$-norm:

$$
\|u\|_{Z_{\theta}(I)} \leq C_{0}\|u\|_{X \frac{d-2}{2}(I)}
$$

for some $C_{0}>0$ independent of $I$.

First, we present the bilinear Strichartz estimate adapted to the $Z_{\theta}$-norm.

Lemma 6.1. Let $N_{1} \leq N_{2}$. Then, we have

$$
\left\|\mathbf{P}_{N_{1}} u_{1} \mathbf{P}_{N_{2}} u_{2}\right\|_{L_{t, x}^{2}\left(I \times \mathbb{R}^{d}\right)} \lesssim\left(\frac{N_{1}}{N_{2}}\right)^{\frac{1}{2}(1-\theta)-}\left\|\mathbf{P}_{N_{1}} u_{1}\right\|_{Z_{\theta}(I)}\left\|\mathbf{P}_{N_{2}} u_{2}\right\|_{Y^{0}(I)} .
$$


Proof. Given a cube $R$ of side length $N_{1}$ centered at $\xi_{0} \in N_{1} \mathbb{Z}^{d}$, let $\mathbf{P}_{R}=\psi\left(\frac{D-\xi_{0}}{N_{1}}\right)$ denote a smooth projection onto $R$ on the frequency side. Here, $\psi$ denotes the smooth projection onto the unit cube defined in (1.6). By (3.10), we have

$$
\begin{aligned}
\left\|\mathbf{P}_{N_{1}} u_{1} \mathbf{P}_{R} \mathbf{P}_{N_{2}} u_{2}\right\|_{L_{t, x}^{2}(I)} & \leq\left\|\mathbf{P}_{N_{1}} u_{1}\right\|_{L_{t, x}^{4}}\left\|\mathbf{P}_{R} \mathbf{P}_{N_{2}} u_{2}\right\|_{L_{t, x}^{4}} \\
& \lesssim N_{1}^{\frac{d-2}{4}}\left\|\mathbf{P}_{N_{1}} u_{1}\right\|_{L_{t, x}^{4}(I)}\left\|\mathbf{P}_{R} \mathbf{P}_{N_{2}} u_{2}\right\|_{Y^{0}(I)} .
\end{aligned}
$$

Then, by almost orthogonality, we have

$$
\begin{aligned}
\left\|\mathbf{P}_{N_{1}} u_{1} \mathbf{P}_{N_{2}} u_{2}\right\|_{L_{t, x}^{2}(I)} \sim\left(\sum_{R}\left\|\mathbf{P}_{N_{1}} u_{1} \mathbf{P}_{R} \mathbf{P}_{N_{2}} u_{2}\right\|_{L_{t, x}^{2}(I)}^{2}\right)^{\frac{1}{2}} \\
\quad \lesssim\left\|\mathbf{P}_{N_{1}} u_{1}\right\|_{Z(I)}\left(\sum_{R}\left\|\mathbf{P}_{R} \mathbf{P}_{N_{2}} u_{2}\right\|_{Y^{0}(I)}^{2}\right)^{\frac{1}{2}} \lesssim\left\|\mathbf{P}_{N_{1}} u_{1}\right\|_{Z(I)}\left\|\mathbf{P}_{N_{2}} u_{2}\right\|_{Y^{0}(I)} .
\end{aligned}
$$

Then, (6.4) follows from interpolating this with (3.6).

Next, we state the key nonlinear estimate. Given $I \subset \mathbb{R}$, we define the $W^{s}$-norm by

$$
\|f\|_{W^{s}(I)}:=\max \left(\left\|\langle\nabla\rangle^{s} f\right\|_{L_{t, x}^{4}(I)},\left\|\langle\nabla\rangle^{s} f\right\|_{L_{t, x}^{d+2}(I)},\left\|\langle\nabla\rangle^{s} f\right\|_{L_{t, x}^{\frac{6(d+2)}{d+4}}(I)}\right) .
$$

As in the proof of Proposition 4.1 different space-time norms of $f$ appear in the estimate but they are all controlled by this $W^{s}$-norm. The following lemma is analogous to Proposition 4.1 but with one important difference. All the terms on the right-hand side have (i) two factors of the $Z_{\theta}$-norm of $v_{j}$, which is weaker than the $X^{s}$-norm, or (ii) the $W^{s}$-norm of $f$, which can be made small by shrinking the interval $I$.

Lemma 6.2. Let $d \geq 3$ and $\theta \in(0,1)$. Suppose that $s, \alpha \in \mathbb{R}$ satisfy

$$
s \in\left(s_{d}, s_{\text {crit }}\right] \quad \text { and } \quad 0<\alpha<1-\frac{2}{d-1} s,
$$

where $s_{d}$ is as in (1.11). Then, given any interval $I=\left[t_{0}, t_{1}\right] \subset \mathbb{R}$, we have

$$
\begin{aligned}
\| \prod_{j=1}^{3}\left(v_{j}+f\right)^{*} & \left\|_{N \frac{d-2}{2}(I)} \lesssim \sum_{j=1}^{3}\right\| v_{j}\left\|_{X} \frac{d-2}{2} \prod_{\substack{k=1 \\
k \neq j}}^{3}\right\| v_{k} \|_{Z_{\theta}(I)} \\
& +\prod_{\substack{j, k=1 \\
j \neq k}}^{3}\left\|v_{j}\right\|_{X^{\frac{d-2}{2}}(I)}\left\|v_{k}\right\|_{X^{\frac{d-2}{2}}(I)}\left(\|f\|_{W^{s}(I)}+\|f\|_{Y^{s}(I)}^{1-\alpha}\|f\|_{W^{s}(I)}^{\alpha}\right) \\
& +\sum_{j=1}^{3}\left\|v_{j}\right\|_{X^{\frac{d-2}{2}}(I)}\left(\|f\|_{Y^{s}(I)}\|f\|_{W^{s}(I)}+\|f\|_{Y^{s}(I)}^{2-2 \alpha}\|f\|_{W^{s}(I)}^{2 \alpha}+\|f\|_{W^{s}(I)}^{2}\right) \\
& +\|f\|_{Y^{s}(I)}\|f\|_{W^{s}(I)}^{2}+\|f\|_{Y^{s}(I)}^{3-2 \alpha}\|f\|_{W^{s}(I)}^{2 \alpha}+\|f\|_{W^{s}(I)}^{3}
\end{aligned}
$$

for all $f \in W^{s}(I) \cap Y^{s}(I)$ and $v_{j} \in X^{\frac{d-2}{2}}(I), j=1,2,3$, where $\left(v_{j}+f\right)^{*}=v_{j}+f$ or $\bar{v}_{j}+\bar{f}$.

We first state and prove the following local well-posedness result for the perturbed NLS (6.1), assuming Lemma 6.2 The proof of Lemma 6.2 is presented at the end of this section. 
Proposition 6.3 (Local well-posedness of the perturbed NLS). Given $d \geq 3$, let $s \in\left(s_{d}, s_{\text {crit }}\right]$, where $s_{d}$ is defined in (1.11). Let $\theta \in\left(\frac{1}{2}, 1\right)$ and $\alpha \in \mathbb{R}$ satisfy (6.6). Suppose that

$$
\left\|v_{0}\right\|_{H^{\frac{d-2}{2}}} \leq R \quad \text { and } \quad\|f\|_{Y^{s}(I)} \leq M
$$

for some $R, M \geq 1$. Then, there exists small $\eta_{0}=\eta_{0}(R, M)>0$ such that if

$$
\left\|S\left(t-t_{0}\right) v_{0}\right\|_{Z_{\theta}(I)} \leq \eta \quad \text { and } \quad\|f\|_{W^{s}(I)} \leq \eta^{\frac{4-\alpha}{\alpha}}
$$

for some $\eta \leq \eta_{0}$ and some time interval $I=\left[t_{0}, t_{1}\right] \subset \mathbb{R}$, then there exists a unique solution $v \in X^{\frac{d-2}{2}}(I) \cap C\left(I ; H^{\frac{d-2}{2}}\left(\mathbb{R}^{d}\right)\right)$ to (6.1) with $v\left(t_{0}\right)=v_{0}$. Moreover, we have

$$
\left\|v-S\left(t-t_{0}\right) v_{0}\right\|_{X}{ }_{\frac{d-2}{2}(I)} \lesssim \eta^{3-2 \theta} .
$$

Proof. For $\theta \in\left(\frac{1}{2}, 1\right)$, we show that the map $\Gamma$ defined by

$$
\Gamma v(t):=S\left(t-t_{0}\right) v_{0}-i \int_{t_{0}}^{t} S\left(t-t^{\prime}\right) \mathcal{N}(v+f)\left(t^{\prime}\right) d t^{\prime}
$$

is a contraction on

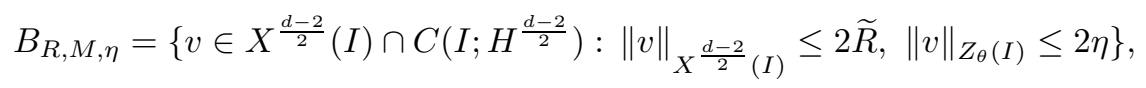

where $\widetilde{R}:=\max (R, M)$. Now, choose

$$
\eta_{0} \ll \widetilde{R}^{-\frac{1}{2 \theta-1}} .
$$

In particular, we have $\eta_{0} \ll \widetilde{R}^{-1} \leq 1$. Fix $\eta \leq \eta_{0}$ in the following. Noting that $\frac{4-\alpha}{\alpha}>3$, Lemma 6.2 with Lemma 3.5 yields

$$
\begin{aligned}
\|\Gamma v\|_{X^{\frac{d-2}{2}(I)}} & \leq\left\|S\left(t-t_{0}\right) v_{0}\right\|_{X^{\frac{d-2}{2}}(I)}+\left\|\Gamma v-S\left(t-t_{0}\right) v_{0}\right\|_{X^{\frac{d-2}{2}}(I)} \\
& \leq\left\|v_{0}\right\|_{H^{\frac{d-2}{2}}}+C \eta^{2} \widetilde{R} \leq 2 \widetilde{R}
\end{aligned}
$$

and

$$
\left\|\Gamma v_{1}-\Gamma v_{2}\right\|_{X \frac{d-2}{2}(I)} \leq \frac{1}{2}\left\|v_{1}-v_{2}\right\|_{X^{\frac{d-2}{2}}(I)}
$$

for $v, v_{1}, v_{2} \in B_{R, M, \eta}$. Moreover, we have

$$
\begin{aligned}
\|\Gamma v\|_{Z_{\theta}(I)} & \leq\left(\left\|S\left(t-t_{0}\right) v_{0}\right\|_{Z(I)}+C \eta^{2} \widetilde{R}\right)^{\theta}\left(\left\|S\left(t-t_{0}\right) v_{0}\right\|_{X}{ }_{X-2}^{\frac{d-2}{2}(I)}+C \eta^{2} \widetilde{R}\right)^{1-\theta} \\
& \leq \eta+C \eta^{2 \theta} \widetilde{R}+C \eta^{2-\theta} \widetilde{R}^{1-\theta}+C \eta^{2} \widetilde{R} \leq 2 \eta
\end{aligned}
$$

for $v \in B_{R, M, \eta}$. Hence, $\Gamma$ is a contraction on $B_{R, M, \eta}$. The estimate (6.7) follows from (6.9) and (6.10).

We conclude this section by presenting the proof of Lemma 6.2. Some cases follow directly from the proof of Proposition 4.1. However, due to the use of the $Z_{\theta}$-norm, we need to make modifications in several cases.

Proof of Lemma 6.2. As in the proof of Proposition 4.1, we need to estimate the right-hand side of (4.10) by performing a case-by-case analysis of expressions of the form:

$$
\left|\iint_{I \times \mathbb{R}^{d}}\langle\nabla\rangle^{\frac{d-2}{2}}\left(w_{1} w_{2} w_{3}\right) v_{4} d x d t\right|,
$$


where $\left\|v_{4}\right\|_{Y^{0}(I)} \leq 1$ and $w_{j}=v$ or $f, j=1,2,3$. Before proceeding further, let us simplify some of the notation. In the following, as before, we drop the complex conjugate sign and denote $X^{s}(I)$ and $Y^{s}(I)$ by $X^{s}$ and $Y^{s}$. Lastly, we dyadically decompose $w_{j}, j=1,2,3$, and $v_{4}$ such that their spatial frequency supports are $\left\{\left|\xi_{j}\right| \sim N_{j}\right\}$ for some dyadic $N_{j} \geq 1$ but still denote them as $w_{j}=v_{j}$ or $f_{j}$, $j=1,2,3$, and $v_{4}$ if there is no confusion.

Case (1): vvv case. Without loss of generality, assume that $N_{1} \geq N_{2}, N_{3}$.

- Subcase (1.a): $N_{1} \sim N_{4}$. By Lemma 6.1, we have

$$
\begin{aligned}
& \left|\iint_{I \times \mathbb{R}^{d}}\langle\nabla\rangle^{\frac{d-2}{2}} v_{1} v_{2} v_{3} v_{4} d x d t\right| \\
& \quad \lesssim \sum_{N_{1} \sim N_{4} \gtrsim N_{2}, N_{3}} N_{1}^{\frac{d-2}{2}}\left\|\mathbf{P}_{N_{1}} v_{1} \mathbf{P}_{N_{3}} v_{3}\right\|_{L_{t, x}^{2}}\left\|\mathbf{P}_{N_{2}} v_{2} \mathbf{P}_{N_{4}} v_{4}\right\|_{L_{t, x}^{2}} \\
& \quad \lesssim \sum_{N_{1}, \ldots, N_{4}}\left(\frac{N_{2} N_{3}}{N_{1} N_{4}}\right)^{\frac{1}{2}(1-\theta)-}\left\|\mathbf{P}_{N_{1}} v_{1}\right\|_{Y^{\frac{d-2}{2}}}\left\|\mathbf{P}_{N_{2}} v_{2}\right\|_{Z_{\theta}}\left\|\mathbf{P}_{N_{3}} v_{3}\right\|_{Z_{\theta}}\left\|\mathbf{P}_{N_{4}} v_{4}\right\|_{Y^{0}}
\end{aligned}
$$

By first summing over $N_{2}, N_{3} \leq N_{1}$ and then applying Cauchy-Schwarz' inequality in summing over $N_{1} \sim N_{4}$, we have

$$
\lesssim\left\|v_{1}\right\|_{X \frac{d-2}{2}(I)}\left\|v_{2}\right\|_{Z_{\theta}(I)}\left\|v_{3}\right\|_{Z_{\theta}(I)} .
$$

- Subcase (1.b): $N_{1} \sim N_{2} \gg N_{4}$. By Lemma 6.1 and (3.5) in Lemma 3.5, we have

$$
\begin{aligned}
& \left|\iint_{I \times \mathbb{R}^{d}}\langle\nabla\rangle^{\frac{d-2}{2}} v_{1} v_{2} v_{3} v_{4} d x d t\right| \\
& \quad \lesssim \sum_{N_{1} \sim N_{2} \geq N_{3}, N_{4}} N_{1}^{\frac{d-2}{2}}\left\|\mathbf{P}_{N_{1}} v_{1} \mathbf{P}_{N_{3}} v_{3}\right\|_{L_{t, x}^{2}}\left\|\mathbf{P}_{N_{2}} v_{2} \mathbf{P}_{N_{4}} v_{4}\right\|_{L_{t, x}^{2}} \\
& \quad \lesssim \sum_{N_{1} \sim N_{2} \geq N_{3}, N_{4}}\left(\frac{N_{3}}{N_{1}}\right)^{\frac{1}{2}(1-\theta)-}\left\|\mathbf{P}_{N_{1}} v_{1}\right\|_{Y} \frac{d-2}{2}\left\|\mathbf{P}_{N_{3}} v_{3}\right\|_{Z_{\theta}}\left\|\mathbf{P}_{N_{2}} v_{2}\right\|_{L_{t, x}^{4}}\left\|\mathbf{P}_{N_{4}} v_{4}\right\|_{L_{t, x}^{4}} \\
& \quad \lesssim \sum_{N_{1} \sim N_{2} \geq N_{3}, N_{4}}\left(\frac{N_{3}}{N_{1}}\right)^{\frac{1}{2}(1-\theta)-}\left(\frac{N_{4}}{N_{2}}\right)^{\frac{d-2}{4}}\left\|\mathbf{P}_{N_{1}} v_{1}\right\|_{Y^{\frac{d-2}{2}}\left\|\mathbf{P}_{N_{3}} v_{3}\right\|_{Z_{\theta}}} \\
& \times N_{2}^{\frac{d-2}{4}}\left\|\mathbf{P}_{N_{2}} v_{2}\right\|_{L_{t, x}^{4}}\left\|\mathbf{P}_{N_{4}} v_{4}\right\|_{Y^{0}} .
\end{aligned}
$$

Summing over $N_{3}$ and taking a supremum in $N_{2}$,

$$
\lesssim\left\|v_{2}\right\|_{Z}\left\|v_{3}\right\|_{Z_{\theta}} \sum_{N_{1} \gg N_{4}}\left(\frac{N_{4}}{N_{1}}\right)^{\frac{d-2}{4}}\left\|\mathbf{P}_{N_{1}} v_{1}\right\|_{Y^{\frac{d-2}{2}}}\left\|\mathbf{P}_{N_{4}} v_{4}\right\|_{Y^{0}}
$$

By Lemma 3.6. we have

$$
\lesssim\left\|v_{1}\right\|_{Y^{\frac{d-2}{2}}}\left\|v_{2}\right\|_{Z_{\theta}}\left\|v_{3}\right\|_{Z_{\theta}}\left\|v_{4}\right\|_{Y^{0}} \lesssim\left\|v_{1}\right\|_{X^{\frac{d-2}{2}}(I)}\left\|v_{2}\right\|_{Z_{\theta}(I)}\left\|v_{3}\right\|_{Z_{\theta}(I)}
$$

In the following, the desired estimates follow from the corresponding cases in the proof of Proposition 4.1. Hence, we just state the results. 
Case (2): $f f f$ case. Without loss of generality, assume $N_{3} \geq N_{2} \geq N_{1}$.

- Subcase (2.a): $N_{2} \sim N_{3}$. The contribution to (6.11) in this case is at most

$$
\lesssim\|f\|_{L_{t, x}^{d+2}}^{2}\left\|\langle\nabla\rangle^{\frac{d-2}{4}+} f\right\|_{L_{t, x}^{4}}^{2} \leq\|f\|_{W^{s}(I)}^{3}
$$

as long as $s>\frac{d-2}{4}$.

- Subcase (2.b): $N_{3} \sim N_{4} \gg N_{1}, N_{2}$.

○ Subsubcase (2.b.i): $N_{1}, N_{2} \ll N_{3}^{\frac{1}{d-1}}$. The contribution to (6.11) in this case is at most

$$
\lesssim\|f\|_{Y^{s}}^{3-2 \alpha}\left\|\langle\nabla\rangle^{s} f\right\|_{L_{t, x}^{4}}^{2 \alpha} \leq\|f\|_{Y^{s}(I)}^{3-2 \alpha}\|f\|_{W^{s}(I)}^{2 \alpha}
$$

as long as (4.13) is satisfied and $\alpha<1-\frac{2}{d-1} s$.

○ Subsubcase (2.b.ii): $N_{2} \gtrsim N_{3}^{\frac{1}{d-1}}$. The contribution to (6.11) in this case is at most

$$
\lesssim\|f\|_{Y^{s}(I)}\left\|\langle\nabla\rangle^{s} f\right\|_{L_{t, x}^{4}}^{2} \leq\|f\|_{Y^{s}(I)}\|f\|_{W^{s}(I)}^{2}
$$

as long as (4.13) is satisfied.

○ Subsubcase (2.b.iii): $N_{1}, N_{2} \gtrsim N_{3}^{\frac{1}{d-1}}$. The contribution to (6.11) in this case is at most

$$
\lesssim\left\|\langle\nabla\rangle^{s} f\right\|_{L_{t, x}^{\frac{6(d+2)}{d+4}}}^{3} \leq\|f\|_{W^{s}(I)}^{3}
$$

as long as (4.13) is satisfied.

Case (3): vvf case. Without loss of generality, assume $N_{1} \geq N_{2}$.

- Subcase (3.a): $N_{1} \gtrsim N_{3}$. The contribution to (6.11) in this case is at most

$$
\lesssim\|v\|_{X \frac{d-2}{2}}^{2}\left\|\langle\nabla\rangle^{s} f\right\|_{L_{t, x}^{d+2}} \leq\|v\|_{X^{\frac{d-2}{2}(I)}}^{2}\|f\|_{W^{s}(I)}
$$

as long as $s>0$.

- Subcase (3.b): $N_{3} \sim N_{4} \gg N_{1} \geq N_{2}$.

- Subsubcase (3.b.i): $N_{1} \gtrsim N_{3}^{\frac{1}{d-1}}$. The contribution to (6.11) in this case is at most

$$
\lesssim\|v\|_{X \frac{d-2}{2}}^{2}\left\|\langle\nabla\rangle^{s} f\right\|_{L_{t, x}^{d+2}} \leq\|v\|_{X^{\frac{d-2}{2}}(I)}^{2}\|f\|_{W^{s}(I)}
$$

as long as (4.14) is satisfied.

○ Subsubcase (3.b.ii): $N_{2} \leq N_{1} \ll N_{3}^{\frac{1}{d-1}}$. The contribution to (6.11) in this case is at most

$$
\lesssim\|v\|_{X^{\frac{d-2}{2}}}^{2}\|f\|_{Y^{s}}^{1-\alpha}\left\|\langle\nabla\rangle^{s} f\right\|_{L_{t, x}^{d+2}}^{\alpha} \leq\|v\|_{X^{\frac{d-2}{2}}(I)}^{2}\|f\|_{Y^{s}(I)}^{1-\alpha}\|f\|_{W^{s}(I)}^{\alpha}
$$

as long as (4.14) is satisfied.

Case (4): vff case. Without loss of generality, assume $N_{3} \geq N_{2}$.

- Subcase (4.a): $N_{1} \gtrsim N_{3}$. The contribution to (6.11) in this case is at most

$$
\lesssim\|v\|_{X \frac{d-2}{2}}\|f\|_{L_{t, x}^{d+2}}^{2} \leq\|v\|_{X \frac{d-2}{2}(I)}\|f\|_{W^{s}(I)}^{2}
$$

as long as $s>0$. 
- Subcase (4.b): $N_{3} \gg N_{1}$. First, suppose that $N_{2} \sim N_{3}$. Then, the contribution to (6.11) in this case is at most

$$
\lesssim\|v\|_{X^{\frac{d-2}{2}}}\left\|\langle\nabla\rangle^{s} f\right\|_{L_{t, x}^{4}}^{2} \leq\|v\|_{X^{\frac{d-2}{2}(I)}}\|f\|_{W^{s}(I)}^{2}
$$

as long as $s>\frac{d-2}{4}$.

Hence, it remains to consider the case $N_{3} \sim N_{4} \gg N_{1}, N_{2}$.

○ Subsubcase (4.b.i): $N_{1}, N_{2} \ll N_{3}^{\frac{1}{d-1}}$. The contribution to (6.11) in this case is at most

$$
\lesssim\|v\|_{X^{\frac{d-2}{2}}}\|f\|_{Y^{s}}^{2-2 \alpha}\left\|\langle\nabla\rangle^{s} f\right\|_{L_{t, x}^{4}}^{2 \alpha} \leq\|v\|_{X^{\frac{d-2}{2}(I)}}\|f\|_{Y^{s}(I)}^{2-2 \alpha}\|f\|_{W^{s}(I)}^{2 \alpha}
$$

as long as (4.17) is satisfied and $\alpha<1-\frac{2}{d-1} s$.

○ Subsubcase (4.b.ii): $N_{1} \ll N_{3}^{\frac{1}{d-1}} \lesssim N_{2}$. The contribution to (6.11) in this case is at most

$$
\lesssim\|v\|_{X^{\frac{d-2}{2}}}\left\|\langle\nabla\rangle^{s} f\right\|_{L_{t, x}^{4}}^{2} \leq\|v\|_{X^{\frac{d-2}{2}}(I)}\|f\|_{W^{s}(I)}^{2}
$$

as long as (4.17) is satisfied.

○ Subsubcase (4.b.iii): $N_{2} \ll N_{3}^{\frac{1}{d-1}} \lesssim N_{1}$. The contribution to (6.11) in this case is at most

$$
\lesssim\|v\|_{X \frac{d-2}{2}}\|f\|_{Y^{s}}\left\|\langle\nabla\rangle^{s} f\right\|_{L_{t, x}^{d+2}} \leq\|v\|_{X^{\frac{d-2}{2}}(I)}\|f\|_{Y^{s}(I)}\|f\|_{W^{s}(I)}
$$

as long as 4.17) is satisfied.

- Subsubcase (4.b.iv): $N_{1}, N_{2} \gtrsim N_{3}^{\frac{1}{d-1}}$. The contribution to (6.11) in this case is at most

$$
\lesssim\|v\|_{X^{\frac{d-2}{2}}}\left\|\langle\nabla\rangle^{s} f\right\|_{L_{t, x}^{d+2}}^{2} \leq\|v\|_{X^{\frac{d-2}{2}}(I)}\|f\|_{W^{s}(I)}^{2}
$$

as long as (4.17) is satisfied.

\section{LONG TIME EXISTENCE OF SOLUTIONS TO THE PERTURBED NLS}

The main goal of this section is to establish long time existence of solutions to the perturbed NLS (6.1) under some assumptions. See Proposition 7.2, We achieve this goal by iteratively applying the perturbation lemma (Lemma 7.1) for the energy-critical NLS.

We first state the perturbation lemma for the energy-critical cubic NLS involving the $X^{\frac{d-2}{2}}$ - and the $Z$-norms. See [19,38,56,57] for perturbation and stability results on usual Strichartz and Lebesgue spaces. In the context of the cubic NLS on $\mathbb{R} \times \mathbb{T}^{3}$, Ionescu-Pausader [31] proved a perturbation lemma involving the critical $X^{s_{\text {crit }}}$ norm. Our proof essentially follows their argument and is included for the sake of completeness.

Lemma 7.1 (Perturbation lemma). Let $d \geq 3$ and let $I$ be a compact interval with $|I| \leq 1$. Suppose that $v \in C\left(I ; H^{\frac{d-2}{2}}\left(\mathbb{R}^{d}\right)\right)$ satisfies the following perturbed $N L S$ :

$$
i \partial_{t} v+\Delta v=|v|^{2} v+e,
$$

satisfying

$$
\|v\|_{Z(I)}+\|v\|_{L^{\infty}\left(I ; H^{\frac{d-2}{2}}\left(\mathbb{R}^{d}\right)\right)} \leq R
$$


for some $R \geq 1$. Then, there exists $\varepsilon_{0}=\varepsilon_{0}(R)>0$ such that if we have

$$
\left\|u_{0}-v\left(t_{0}\right)\right\|_{H^{\frac{d-2}{2}}\left(\mathbb{R}^{d}\right)}+\|e\|_{N^{\frac{d-2}{2}}(I)} \leq \varepsilon
$$

for some $u_{0} \in H^{\frac{d-2}{2}}\left(\mathbb{R}^{d}\right)$, some $t_{0} \in I$, and some $\varepsilon<\varepsilon_{0}$, then there exists a solution $u \in X^{\frac{d-2}{2}}(I) \cap C\left(I ; H^{\frac{d-2}{2}}\left(\mathbb{R}^{d}\right)\right)$ to the defocusing cubic NLS (1.1) with $u\left(t_{0}\right)=u_{0}$ such that

$$
\begin{aligned}
& \|u\|_{X \frac{d-2}{2}(I)}+\|v\|_{X \frac{d-2}{2}(I)} \leq C(R), \\
& \|u-v\|_{X}{ }_{\frac{d-2}{2}(I)} \leq C(R) \varepsilon,
\end{aligned}
$$

where $C(R)$ is a nondecreasing function of $R$.

Proof. Without loss of generality, we assume $t_{0}=\min I$. Given small $\varepsilon_{1}=\varepsilon_{1}(R)>$ 0 (to be chosen later), we divide the interval $I$ into subintervals $I_{j}=\left[t_{j}, t_{j+1}\right]$ such that $I=\bigcup_{j=0}^{L} I_{j}$. By choosing $L \sim\left(\frac{R}{\varepsilon_{1}}\right)^{4}$, we can guarantee that

$$
\|v\|_{Z\left(I_{j}\right)} \leq \varepsilon_{1}
$$

for $j=0, \ldots, L$. By assumption, we also have

$$
\|e\|_{N} \frac{d-2}{2}\left(I_{j}\right) \leq \varepsilon<\varepsilon_{0}
$$

for $j=0, \ldots, L$.

Step 1. Let $\theta \in\left(\frac{1}{2}, 1\right)$. We first claim that there exist $\eta_{0}=\eta_{0}(R)>0$ and $\varepsilon_{0}=\varepsilon_{0}(R)>0$ such that if

$$
\left\|S\left(t-t_{*}\right) v\left(t_{*}\right)\right\|_{Z_{\theta}(J)} \leq \eta_{0} \quad \text { and } \quad\|e\|_{N^{\frac{d-2}{2}}(J)} \leq \varepsilon_{0}
$$

for some $t_{*}$ in a subinterval $J \subset I$, then there exists a unique solution $v$ to (7.1) on $J$, satisfying

$$
\left\|v-S\left(t-t_{*}\right) v\left(t_{*}\right)\right\|_{X^{\frac{d-2}{2}}(J)} \leq C\left\|S\left(t-t_{*}\right) v\left(t_{*}\right)\right\|_{Z_{\theta}(J)}^{3-2 \theta}+2\|e\|_{N^{\frac{d-2}{2}}(J)} .
$$

We choose $\eta_{0}=\eta_{0}(R)$ and $\varepsilon_{0}=\varepsilon_{0}(R)$ such that

$$
\eta_{0} \ll R^{-\frac{1}{2 \theta-1}} \quad \text { and } \quad \varepsilon_{0} \ll R^{-\frac{2}{2 \theta-1}} .
$$

In the following, we set

$$
\eta:=\left\|S\left(t-t_{*}\right) v\left(t_{*}\right)\right\|_{Z_{\theta}(J)} \leq \eta_{0} \quad \text { and } \quad \varepsilon:=\|e\|_{N^{\frac{d-2}{2}(J)}} \leq \varepsilon_{0} .
$$

Then, proceeding as in the proof of Proposition 6.3. we show that the map $\Gamma$ defined by

$$
\Gamma v(t):=S\left(t-t_{*}\right) v\left(t_{*}\right)-i \int_{t_{*}}^{t} S\left(t-t^{\prime}\right) \mathcal{N}(v)\left(t^{\prime}\right) d t^{\prime}-i \int_{t_{*}}^{t} S\left(t-t^{\prime}\right) e\left(t^{\prime}\right) d t^{\prime}
$$

is a contraction on

$$
B_{R, \eta, \varepsilon}=\left\{v \in X^{\frac{d-2}{2}}(J) \cap C\left(J ; H^{\frac{d-2}{2}}\right):\|v\|_{X^{\frac{d-2}{2}}(J)} \leq 2 R,\|v\|_{Z_{\theta}(J)} \leq 2\left(\eta+\varepsilon^{\frac{\theta}{2}+\frac{1}{4}}\right)\right\} .
$$

Indeed, by Lemma 6.2 (with $f=0$ ), we have

$$
\begin{aligned}
\|\Gamma v\|_{X} \frac{d-2}{2}(J) & \leq\left\|v\left(t_{*}\right)\right\|_{H^{\frac{d-2}{2}}}+C\left(\eta+\varepsilon^{\frac{\theta}{2}+\frac{1}{4}}\right)^{2} R+\varepsilon \\
& \leq\left\|v\left(t_{*}\right)\right\|_{H^{\frac{d-2}{2}}}+C \eta^{2} R+2 \varepsilon \leq 2 R
\end{aligned}
$$


and

$$
\left\|\Gamma v_{1}-\Gamma v_{2}\right\|_{X \frac{d-2}{2}(J)} \leq C\left(\eta+\varepsilon^{\frac{\theta}{2}+\frac{1}{4}}\right) R\left\|v_{1}-v_{2}\right\|_{X \frac{d-2}{2}(J)} \leq \frac{1}{2}\left\|v_{1}-v_{2}\right\|_{X^{\frac{d-2}{2}}(J)}
$$

for $v, v_{1}, v_{2} \in B_{R, \eta, \varepsilon}$. Moreover, we have

$$
\begin{aligned}
\|\Gamma v\|_{Z_{\theta}(J)} \leq\left(\| S\left(t-t_{*}\right)\right. & \left.v\left(t_{*}\right) \|_{Z(J)}+C \eta^{2} R+\varepsilon\right)^{\theta} \\
& \times\left(\left\|S\left(t-t_{*}\right) v\left(t_{*}\right)\right\|_{X^{\frac{d-2}{2}(J)}}+C \eta^{2} R+\varepsilon\right)^{1-\theta} \\
\leq \eta+C \eta^{2 \theta} R & +C \eta^{2-\theta} R^{1-\theta}+C \eta^{\theta} \varepsilon^{1-\theta}+C \varepsilon^{\theta} R^{1-\theta} \leq 2\left(\eta+\varepsilon^{\frac{\theta}{2}+\frac{1}{4}}\right)
\end{aligned}
$$

for $v \in B_{R, \eta, \varepsilon}$. Hence, $\Gamma$ is a contraction on $B_{R, \eta_{1}}$. The estimate (7.9) follows from (7.10) and (7.12).

Step 2. Next, we claim that, given $\varepsilon_{2}>0$, we can choose $\varepsilon_{j}=\varepsilon_{j}\left(R, \varepsilon_{2}\right), j=0,1$, in (7.7) and (7.6) sufficiently small such that we have

$$
\left\|S\left(t-t_{j}\right) v\left(t_{j}\right)\right\|_{Z_{\theta}\left(I_{j}\right)} \leq \varepsilon_{2} \quad \text { and } \quad\|v\|_{Z_{\theta}\left(I_{j}\right)} \leq \varepsilon_{2} .
$$

Without loss of generality, assume $\varepsilon_{2} \leq \frac{\eta_{0}}{2}$, where $\eta_{0}=\eta_{0}(R)$ is as in Step 1 . Let $h(\tau)=\left\|S\left(t-t_{j}\right) v\left(t_{j}\right)\right\|_{Z_{\theta}\left(\left[t_{j}, t_{j}+\tau\right]\right)}$. Note that $h$ is continuous and $h(0)=0$. Thus, we have $h(\tau) \leq 2 \varepsilon_{2} \leq \eta_{0}$ for small $\tau>0$. Then, from the Duhamel formula (7.11) with (7.6), (7.7), and (7.9), we have

$$
\begin{aligned}
h(\tau) & \leq\left\|S\left(t-t_{j}\right) v\left(t_{j}\right)\right\|_{X^{\frac{d-2}{2}}}^{1-\theta}\left(\left[t_{j}, t_{j}+\tau\right]\right) \\
& \leq C R^{1-\theta}\left(\varepsilon_{1}+\varepsilon_{2}^{3-2 \theta}+\|e\|_{N^{\frac{d-2}{2}}\left(I_{j}\right)}\right)^{\theta} \\
& \leq C R^{1-\theta} \varepsilon_{2}^{\theta(3-2 \theta)}+C R^{1-\theta}\left(\varepsilon_{1}+\|e\|_{N^{\frac{d-2}{2}}\left(I_{j}\right)}\right)^{\theta} .
\end{aligned}
$$

From (17.10) with $\varepsilon_{2} \leq \frac{\eta_{0}}{2}$, we have

$$
C R^{1-\theta} \varepsilon_{2}^{\theta(3-2 \theta)} \leq C\left(R \eta_{0}^{(2 \theta-1)}\right)^{1-\theta} \varepsilon_{2} \ll \varepsilon_{2} .
$$

Hence, it follows from (7.14) and (7.15) that

$$
h(\tau) \leq \frac{1}{2} \varepsilon_{2}+C R^{1-\theta}\left(\varepsilon_{1}+\varepsilon_{0}\right)^{\theta} \leq \varepsilon_{2}
$$

by choosing $\varepsilon_{j}=\varepsilon_{j}\left(R, \varepsilon_{2}\right)>0$ sufficiently small, $j=0,1$. Then, by the continuity argument, we see that (7.16) holds for all $\tau \leq t_{j+1}-t_{j}$. From Step 1 and (7.6), we have

$$
\|v\|_{Z_{\theta}\left(I_{j}\right)}=\|v\|_{Z\left(I_{j}\right)}^{\theta}\|v\|_{X^{\frac{d-2}{2}}\left(I_{j}\right)}^{1-\theta} \leq C \varepsilon_{1}^{\theta} R^{1-\theta} .
$$

Therefore, (7.13) follows from (7.16) and (7.17), by choosing $\varepsilon_{1}=\varepsilon_{1}\left(R, \varepsilon_{2}\right)$ smaller if necessary.

Step 3. Given $\varepsilon_{2}=\varepsilon_{2}(R)>0$ (to be chosen later), it follows from Step 2 that (7.13) holds as long as $\varepsilon_{j}=\varepsilon_{j}(R)>0, j=0,1$, are sufficiently small. From Step 1 with (7.7), (7.13), and (17.12), we have

$$
\|v\|_{X}{ }_{\frac{d-2}{2}\left(I_{j}\right)} \leq 2 R
$$

as long as $\varepsilon_{j}=\varepsilon_{j}(R)>0, j=0,1,2$, are sufficiently small. 
Let $u$ be a solution to the defocusing cubic NLS (1.1) with initial data $u\left(t_{j}\right)$ given at $t=t_{j}$ such that

$$
\left\|u\left(t_{j}\right)-v\left(t_{j}\right)\right\|_{H^{\frac{d-2}{2}}} \leq \varepsilon<\varepsilon_{0} .
$$

Let $J_{j}=\left[t_{j}, t_{j}+\tau\right] \subset I_{j}$ be the maximal time interval such that

$$
\|u-v\|_{Z_{\theta}\left(J_{j}\right)} \leq 6 C_{0} \varepsilon,
$$

where $C_{0}$ is as in (6.3). Such an interval exists and is nonempty, since $\tau \mapsto$ $\|u-v\|_{Z_{\theta}\left(t_{j}, t_{j}+\tau\right)}$ is finite and continuous (see Lemma A.8), at least on the interval of local existence of $u$, and vanishes for $\tau=0$.

Let $w:=u-v$. By Lemma 6.2 (with $f=0$ ) with (7.7), (7.13), (7.18), (17.19), and (7.20), we have

$$
\begin{aligned}
& \|w\|_{X^{\frac{d-2}{2}}\left(J_{j}\right)} \leq\left\|u\left(t_{j}\right)-v\left(t_{j}\right)\right\|_{H^{\frac{d-2}{2}}}+C_{1}\left\{\|v\|_{X^{\frac{d-2}{2}}\left(J_{j}\right)}\|v\|_{Z_{\theta}\left(J_{j}\right)}\|w\|_{X^{\frac{d-2}{2}}\left(J_{j}\right)}\right.
\end{aligned}
$$

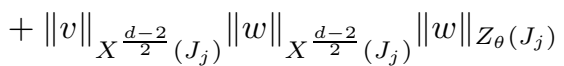

$$
\begin{aligned}
& \left.+\|w\|_{X \frac{d-2}{2}\left(J_{j}\right)}\|w\|_{Z_{\theta}\left(J_{j}\right)}^{2}\right\}+\|e\|_{N^{\frac{d-2}{2}}\left(J_{j}\right)} \\
& \leq 2 \varepsilon+C_{2}\left(\varepsilon_{0}+\varepsilon_{2}\right) R\|w\|_{X} \frac{d-2}{2}\left(J_{j}\right) .
\end{aligned}
$$

Taking $\varepsilon_{j}=\varepsilon_{j}(R)>0$ sufficiently small, $j=0,2$, such that $\left(\varepsilon_{0}+\varepsilon_{2}\right) R \ll 1$, we obtain

$$
\|w\|_{X}{ }_{\frac{d-2}{2}\left(J_{j}\right)} \leq 4 \varepsilon
$$

Hence, from (6.3), we have

$$
\|w\|_{Z_{\theta}\left(J_{j}\right)} \leq C_{0}\|w\|_{X^{\frac{d-2}{2}}\left(J_{j}\right)} \leq 4 C_{0} \varepsilon .
$$

From (7.18) and (7.21), we have $\|u\|_{X^{\frac{d-2}{2}\left(J_{j}\right)}} \leq 3 R<\infty$. Then, from (3.4), we have $\|u\|_{\dot{S}^{\frac{d-2}{2}}\left(J_{j}\right)}<\infty$. In particular, this implies that $u$ can be extended to some larger interval $J^{\prime} \supset \overline{J_{j}}$. Therefore, in view of (7.20) and (7.22), we can apply the continuity argument and conclude that $J_{j}=I_{j}$.

Step 4. By (7.3), we have $\left\|u\left(t_{0}\right)-v\left(t_{0}\right)\right\|_{H^{\frac{d-2}{2}}} \leq \varepsilon$ for some $\varepsilon<\varepsilon_{0}$. Then, by Step 3 , we have $\|w\|_{X \frac{d-2}{2}\left(I_{0}\right)} \leq 4 \varepsilon$ on $I_{0}=\left[t_{0}, t_{1}\right]$. In particular, this yields

$$
\left\|u\left(t_{1}\right)-v\left(t_{1}\right)\right\|_{H^{\frac{d-2}{2}}} \leq 4 C \varepsilon .
$$

Then, we can apply Step 3 on the interval $I_{1}$ by choosing $\varepsilon_{0}$ (and hence $\varepsilon$ ) even smaller. We argue recursively for each interval $I_{j}, j=2, \ldots, L$. Note that, at each step, we make $\varepsilon_{0}$ smaller by a factor of $(4 C)^{-1}$. Since $L \sim\left(\frac{R}{\varepsilon_{1}}\right)^{4}$ and $\varepsilon_{1}=\varepsilon_{1}(R)$, there are a finite number of iterative steps depending only on $R$. This allows us to choose new $\varepsilon_{0}=\varepsilon_{0}(R)>0$ such that, by Lemma A.4 we have

$$
\begin{aligned}
\|u\|_{X \frac{d-2}{2}(I)}+\|v\|_{X^{\frac{d-2}{2}}(I)} & \lesssim L R \lesssim C(R), \\
\|u-v\|_{X^{\frac{d-2}{2}}(I)} & \lesssim L \varepsilon \lesssim C(R) \varepsilon .
\end{aligned}
$$

This completes the proof of Lemma 7.1 
In the remaining part of this section, we consider long time existence of solutions to the perturbed NLS (6.1) under several assumptions. Given $T>0$, we assume that there exist $\beta, C, M>0$ such that

$$
\|f\|_{W^{s}(I)} \leq C|I|^{\beta} \quad \text { and } \quad\|f\|_{Y^{s}([0, T])} \leq M
$$

for any interval $I \subset[0, T]$. Then, Proposition 6.3 guarantees existence of a solution to the perturbed NLS (6.1), at least for a short time.

Proposition 7.2. Let $d \geq 3$. Let $s \in\left(s_{d}, s_{\text {crit }}\right]$, where $s_{d}$ is defined in (1.11). Given $T>0$, assume the following conditions (i) - (iii):

(i) Hypothesis (B) holds if $d \neq 4$.

(ii) $f \in Y^{s}([0, T]) \cap W^{s}([0, T])$ satisfies (7.23) .

(iii) Given a solution $v$ to (6.1), the following a priori bound holds:

$$
\|v\|_{L^{\infty}\left([0, T] ; H^{\frac{d-2}{2}}\left(\mathbb{R}^{d}\right)\right)} \leq R
$$

for some $R>0$.

Then, there exists $\tau=\tau(R, M, T, s, \beta)>0$ such that, given any $t_{0} \in[0, T)$, the solution $v$ to (6.1) exists on $\left[t_{0}, t_{0}+\tau\right] \cap[0, T]$. In particular, condition (iii) guarantees existence of $v$ on the entire interval $[0, T]$.

Remark 7.3. We point out that the first condition in (7.23) can be weakened as follows. Let $\tau=\tau(R, M, T, s, \beta)>0$ be as in Proposition 7.2. Then, it follows from the proof of Proposition 7.2 (see (17.27) and (7.28) below) that if we assume that

$$
\|f\|_{W^{s}\left(\left[t_{0}, t_{0}+\tau_{*}\right)\right)} \leq C\left|\tau_{*}\right|^{\beta}
$$

for some $\tau_{*} \leq \tau$ instead of the first condition in (7.23), then the conclusion of Proposition 7.2 still holds on $\left[t_{0}, t_{0}+\tau_{*}\right] \cap[0, T]$. Indeed, we use this version of Proposition 7.2 in Section 8

Proof. By setting $e=|v+f|^{2}(v+f)-|v|^{2} v$, (6.1) reduces to (7.1). In the following, we iteratively apply Lemma 7.1 on short intervals and show that there exists $\tau=$ $\tau(R, M, T, s, \beta)>0$ such that (7.1) is well-posed on $\left[t_{0}, t_{0}+\tau\right] \cap[0, T]$ for any $t_{0} \in[0, T)$.

Let $w$ be the global solution to the defocusing cubic NLS (1.1) with $w\left(t_{0}\right)=$ $v\left(t_{0}\right)=v_{0}$. By (7.24), we have $\left\|w\left(t_{0}\right)\right\|_{H^{\frac{d-2}{2}}} \leq R$. Then, by Hypothesis (B), we have

$$
\|w\|_{L_{t, x}^{d+2}([0, T])} \leq C(R, T)<\infty .
$$

By the standard argument, this implies that $\left\||\nabla|^{\frac{d-2}{2}} w\right\|_{L_{t}^{q} L_{x}^{r}([0, T])} \leq C^{\prime}(R, T)<\infty$ for all Schrödinger admissible pairs $(q, r)$. In particular, we have $\|w\|_{Z([0, T])} \leq$ $C^{\prime \prime}(R, T)<\infty$ and

$$
\begin{aligned}
\|w\|_{X^{\frac{d-2}{2}}([0, T])} & \leq\left\|v_{0}\right\|_{H^{\frac{d-2}{2}}}+\left\||\nabla|^{\frac{d-2}{2}} w\right\|_{L_{t, x}^{\frac{2(d+2)}{d}}([0, T])}\|w\|_{L_{t, x}^{d+2}([0, T])}^{2} \\
& \leq C^{\prime \prime \prime}(R, T)<\infty .
\end{aligned}
$$

Let $\theta \in\left(\frac{1}{2}, 1\right)$. Given small $\eta>0$ (to be chosen later), we divide the interval $\left[t_{0}, T\right]$ into $J=J(R, T, \eta)$ many subintervals $I_{j}=\left[t_{j}, t_{j+1}\right]$ such that

$$
\|w\|_{Z_{\theta}\left(I_{j}\right)} \sim \eta \text {. }
$$

In the following, we fix the value of $\theta$ and suppress dependence of various constants such as $\tau$ and $\eta$ on $\theta$. 
Fix $\tau>0$ (to be chosen later in terms of $R, M, T, s$, and $\beta$ ) and write $\left[t_{0}, t_{0}+\tau\right]=$ $\bigcup_{j=0}^{J^{\prime}}\left(\left[t_{0}, t_{0}+\tau\right] \cap I_{j}\right)$ for some $J^{\prime} \leq J-1$, where $\left[t_{0}, t_{0}+\tau\right] \cap I_{j} \neq \emptyset$ for $0 \leq j \leq J^{\prime}$ and $\left[t_{0}, t_{0}+\tau\right] \cap I_{j}=\emptyset$ for $j \geq J^{\prime}$.

Since the nonlinear evolution $w$ is small on each $I_{j}$, it follows that the linear evolution $S\left(t-t_{j}\right) w\left(t_{j}\right)$ is also small on each $I_{j}$. Indeed, from the Duhamel formula, we have

$$
S\left(t-t_{j}\right) w\left(t_{j}\right)=w(t)+i \int_{t_{j}}^{t} S\left(t-t^{\prime}\right)|w|^{2} w\left(t^{\prime}\right) d t^{\prime} .
$$

Then, from Case (1) in the proof of Lemma 6.2 with (7.25), we have

$$
\left\|S\left(t-t_{j}\right) w\left(t_{j}\right)\right\|_{Z_{\theta}\left(I_{j}\right)} \leq\|w\|_{Z_{\theta}\left(I_{j}\right)}+C\|w\|_{X^{\frac{d-2}{2}\left(I_{j}\right)}}\|w\|_{Z_{\theta}\left(I_{j}\right)}^{2} \leq \eta+C(R, T) \eta^{2} .
$$

By taking $\eta=\eta(R, T)>0$ sufficiently small, we have

$$
\left\|S\left(t-t_{j}\right) w\left(t_{j}\right)\right\|_{Z_{\theta}\left(I_{j}\right)} \leq 2 \eta
$$

for all $j=0, \ldots, J-1$.

Now, we estimate $v$ on the first interval $I_{0}$. Let $\eta_{0}=\eta_{0}(R, M)$ be as in Proposition 6.3. Then, by Lemma 3.5 (i), (7.24), and Proposition 6.3, we have

$$
\begin{aligned}
\|v\|_{X \frac{d-2}{2}\left(I_{0}\right)} & \leq\left\|S\left(t-t_{0}\right) v\left(t_{0}\right)\right\|_{X \frac{d-2}{2}\left(I_{0}\right)}+\left\|v-S\left(t-t_{0}\right) v\left(t_{0}\right)\right\|_{X}{ }^{\frac{d-2}{2}}\left(I_{0}\right) \\
& \leq R+C \eta^{3-2 \theta} \leq 2 R,
\end{aligned}
$$

as long as $2 \eta<\eta_{0}$ and $\tau=\tau(\eta, \alpha, \beta)=\tau(R, M, T, \alpha, \beta)>0$ is sufficiently small so that

$$
\|f\|_{W^{s}\left(\left[t_{0}, t_{0}+\tau\right)\right)} \leq C \tau^{\beta} \leq \eta^{\frac{4-\alpha}{\alpha}},
$$

where $\alpha=\alpha(s)$ satisfies (6.6).

Next, we estimate the error term. By Lemma 6.2 with (7.23), we have

$$
\|e\|_{N} \frac{d-2}{2}\left(I_{0}\right) \leq C(R, M) \tau^{\alpha \beta} .
$$

Given $\varepsilon>0$, we can choose $\tau=\tau(R, M, T, \varepsilon, \alpha, \beta)>0$ sufficiently small so that

$$
\|e\|_{N \frac{d-2}{2}\left(I_{0}\right)} \leq \varepsilon \text {. }
$$

In particular, for $\varepsilon<\varepsilon_{0}$ with $\varepsilon_{0}=\varepsilon_{0}(R)>0$ dictated by Lemma 7.1 the condition (17.3) is satisfied on $I_{0}$.

Therefore, all the conditions of Lemma 7.1 are satisfied on the first interval $I_{0}$, provided that $\tau=\tau(R, M, T, \varepsilon, \alpha, \beta)>0$ is chosen sufficiently small. Hence, we obtain

$$
\|w-v\|_{X \frac{d-2}{2}\left(I_{0}\right)} \leq C_{0}(R) \varepsilon .
$$

In particular, we have

$$
\left\|w\left(t_{1}\right)-v\left(t_{1}\right)\right\|_{H^{\frac{d-2}{2}}} \leq C_{1}(R) \varepsilon .
$$

Then, from (7.26) and Lemma 3.5 (i) with (7.30), we have

$$
\begin{aligned}
\left\|S\left(t-t_{1}\right) v\left(t_{1}\right)\right\|_{Z_{\theta}\left(I_{1}\right)} & \leq\left\|S\left(t-t_{1}\right) w\left(t_{1}\right)\right\|_{Z_{\theta}\left(I_{1}\right)}+\left\|S\left(t-t_{1}\right)\left(w\left(t_{1}\right)-v\left(t_{1}\right)\right)\right\|_{Z_{\theta}\left(I_{1}\right)} \\
& \leq 2 \eta+C_{1}^{\prime}(R) \varepsilon \leq 3 \eta
\end{aligned}
$$

by choosing $\varepsilon=\varepsilon(R, \eta)>0$ sufficiently small. 
Proceeding as before, it follows from Proposition 6.3 with (7.24) and (7.26) that

$$
\|v\|_{X \frac{d-2}{2}\left(I_{1}\right)} \leq R+C \eta^{3-2 \theta} \leq 2 R,
$$

as long as $3 \eta \leq \eta_{0}$ and $\tau>0$ is sufficiently small so that (7.27) is satisfied. Similarly, it follows from Lemma 6.2 with (7.23) that

$$
\|e\|_{N \frac{d-2}{2}\left(I_{1}\right)} \leq C(R, M) \tau^{\alpha \beta} \leq \varepsilon
$$

by choosing $\tau=\tau(R, M, T, \varepsilon, \alpha, \beta)>0$ sufficiently small. Therefore, all the conditions of Lemma 7.1 are satisfied on the second interval $I_{1}$, provided that $\tau=\tau(R, M, T, \varepsilon, \alpha, \beta)$ is chosen sufficiently small and that $\left(C_{1}(R)+1\right) \varepsilon<\varepsilon_{0}$. Hence, by Lemma 7.1, we obtain

$$
\|w-v\|_{X \frac{d-2}{2}\left(I_{1}\right)} \leq C_{0}(R)\left(C_{1}(R)+1\right) \varepsilon .
$$

In particular, we have

$$
\left\|w\left(t_{2}\right)-v\left(t_{2}\right)\right\|_{H^{\frac{d-2}{2}}} \leq C_{2}(R) \varepsilon .
$$

By choosing $\eta=\eta(R, M, T)>0$ and $\tau=\tau(R, M, T, \varepsilon, \alpha, \beta)>0$ sufficiently small, we can argue inductively and obtain

$$
\left\|w\left(t_{j}\right)-v\left(t_{j}\right)\right\|_{H \frac{d-2}{2}} \leq C_{j}(R) \varepsilon
$$

for all $0 \leq j \leq J^{\prime}$, as long as (i) $\left(J^{\prime}+2\right) \eta \leq \eta_{0}$ and (ii) $\varepsilon=\varepsilon(R, \eta, J)$ is sufficiently small such that $\left(C_{j}(R)+1\right) \varepsilon<\varepsilon_{0}, j=1, \ldots, J^{\prime}$. Recalling that $J^{\prime}+1 \leq J=$ $J(R, T, \eta)$, we see that this can be achieved by choosing $\eta=\eta(R, M, T)>0$, $\varepsilon=\varepsilon(R, M, T)>0$, and $\tau=\tau(R, M, T, \alpha, \beta)=\tau(R, M, T, s, \beta)>0$ sufficiently small. This guarantees existence of the solution $v$ to (7.1) on $\left[t_{0}, t_{0}+\tau\right]$.

Under the conditions (i) - (iii), we can apply the above local argument on time intervals of length $\tau=\tau(R, M, T, s, \beta)>0$, thus extending the solution $v$ to (6.1) on the entire interval $[0, T]$.

\section{Proof of Theorem 1.3}

In this section, we prove the following "almost" almost sure global existence result.

Proposition 8.1. Let $d \geq 3$ and $s \in\left(s_{d}, s_{\text {crit }}\right]$. Assume Hypothesis (A). Furthermore, assume Hypothesis (B) if $d \neq 4$. Given $\phi \in H^{s}\left(\mathbb{R}^{d}\right)$, let $\phi^{\omega}$ be its Wiener randomization defined in (1.9), satisfying (1.10). Then, given any $T, \varepsilon>0$, there exists a set $\widetilde{\Omega}_{T, \varepsilon} \subset \Omega$ such that:

(i) $P\left(\widetilde{\Omega}_{T, \varepsilon}^{c}\right)<\varepsilon$.

(ii) For each $\omega \in \widetilde{\Omega}_{T, \varepsilon}$, there exists a (unique) solution $u$ to (1.1) on $[0, T]$ with $\left.u\right|_{t=0}=\phi^{\omega}$.

It is easy to see that "almost" almost sure global existence implies almost sure global existence. See 20. For completeness, we first show how Theorem 1.3 follows as an immediate consequence of Proposition 8.1 .

Given small $\varepsilon>0$, let $T_{j}=2^{j}$ and $\varepsilon_{j}=2^{-j} \varepsilon, j \in \mathbb{N}$. For each $j$, we apply Proposition 8.1 and construct $\widetilde{\Omega}_{T_{j}, \varepsilon_{j}}$. Then, let $\Omega_{\varepsilon}=\bigcap_{j=1}^{\infty} \widetilde{\Omega}_{T_{j}, \varepsilon_{j}}$. Note that (i) $P\left(\Omega_{\varepsilon}^{c}\right)<\varepsilon$, and (ii) for each $\omega \in \Omega_{\varepsilon}$, we have a global solution $u$ to (1.1) with $\left.u\right|_{t=0}=\phi^{\omega}$. Now, let $\Sigma=\bigcup_{\varepsilon>0} \Omega_{\varepsilon}$. Then, we have $P\left(\Sigma^{c}\right)=0$. Moreover, for each 
$\omega \in \Sigma$, we have a global solution $u$ to (1.1) with $\left.u\right|_{t=0}=\phi^{\omega}$. This proves Theorem 1.3 .

The rest of this section is devoted to the proof of Proposition 8.1.

Proof of Proposition 8.1. Given $T, \varepsilon>0$, set

$$
M=M(T, \varepsilon) \sim\|\phi\|_{H^{s}}\left(\log \frac{1}{\varepsilon}\right)^{\frac{1}{2}} .
$$

Defining $\Omega_{1}=\Omega_{1}(T, \varepsilon)$ by

$$
\Omega_{1}:=\left\{\omega \in \Omega:\left\|S(t) \phi^{\omega}\right\|_{Y^{s}([0, T])} \leq M\right\},
$$

it follows from Lemma 3.5 (i) and Lemma 2.4 that

$$
P\left(\Omega_{1}^{c}\right)<\frac{\varepsilon}{3}
$$

Given $T, \varepsilon>0$, let $R=R\left(T, \frac{\varepsilon}{3}\right)$ and let $M$ be as in (1.13) and (8.1), respectively. With $\tau=\tau(R, M, T)$ as in Proposition 7.2, write

$$
[0, T]=\bigcup_{j=0}^{\left[\frac{T}{\tau_{*}}\right]}\left[j \tau_{*},(j+1) \tau_{*}\right] \cap[0, T]
$$

for some $\tau_{*} \leq \tau$ (to be chosen later). Now, define $\Omega_{2}$ by

$$
\Omega_{2}=\left\{\omega \in \Omega:\left\|S(t) \phi^{\omega}\right\|_{W^{s}\left(\left[j \tau_{*},(j+1) \tau_{*}\right]\right)} \leq C \tau_{*}^{\frac{1}{2(d+2)}}, j=0, \ldots,\left[\frac{T}{\tau_{*}}\right]\right\},
$$

where $C$ is as in (7.23). Then, by Lemma 2.2, we have

$$
P\left(\Omega_{2}^{c}\right) \leq \sum_{j=0}^{\left[\frac{T}{\tau_{*}}\right]} P\left(\left\|S(t) \phi^{\omega}\right\|_{W^{s}\left(\left[j \tau_{*},(j+1) \tau_{*}\right]\right)} \geq C \tau_{*}^{\frac{1}{2(d+2)}}\right) \lesssim \frac{T}{\tau_{*}} \exp \left(-\frac{c}{\tau_{*}^{\frac{1}{d+2}}\|\phi\|_{H^{s}}^{2}}\right) .
$$

By making $\tau_{*}=\tau_{*}\left(\|\phi\|_{H^{s}}\right)$ smaller, if necessary, we have

$$
\lesssim \frac{T}{\tau_{*}} \cdot \tau_{*} \exp \left(-\frac{c}{2 \tau_{*}^{\frac{1}{d+2}}\|\phi\|_{H^{s}}^{2}}\right)=T \exp \left(-\frac{1}{2 \tau_{*}^{\frac{1}{d+2}}\|\phi\|_{H^{s}}^{2}}\right) .
$$

Hence, by choosing $\tau_{*}=\tau_{*}\left(T, \varepsilon,\|\phi\|_{H^{s}}\right)$ sufficiently small, we have

$$
P\left(\Omega_{2}^{c}\right)<\frac{\varepsilon}{3} .
$$

Finally, set $\widetilde{\Omega}_{T, \varepsilon}=\Omega_{T, \frac{\varepsilon}{3}} \cap \Omega_{1} \cap \Omega_{2}$, where $\Omega_{T, \frac{\varepsilon}{3}}$ is as in Hypothesis (A) with $\varepsilon$ replaced by $\frac{\varepsilon}{3}$. Then, from (8.2) and (8.3), we have

$$
P\left(\widetilde{\Omega}_{T, \varepsilon}^{c}\right)<\varepsilon .
$$

Moreover, for $\omega \in \widetilde{\Omega}_{T, \varepsilon}$, we can iteratively apply Proposition 7.2 and Remark 7.3 and construct the solution $v=v^{\omega}$ on each $\left[j \tau_{*},(j+1) \tau_{*}\right], j=0, \ldots,\left[\frac{T}{\tau_{*}}\right]-1$, and $\left[\left[\frac{T}{\tau_{*}}\right] \tau, T\right]$. This completes the proof of Proposition 8.1.

Remark 8.2. It is worthwhile to mention that the proof of Proposition 8.1 strongly depends on the quasi-invariance property of the distribution of the linear solution $S(t) \phi^{\omega}$. More precisely, in the proof above, we exploited the fact that the distribution of $\left\|S(t) \phi^{\omega}\right\|_{W^{s}\left(\left[t_{0}, t_{0}+\tau_{*}\right]\right)}$ depends basically only on the length $\tau_{*}$ of the interval, but is independent of $t_{0}$. 


\section{Probabilistic global existence via Randomization on Dilated Cubes}

In this section, we present the proof of Theorem 1.4. The main idea is to exploit the dilation symmetry of the cubic NLS (1.1). For a function $\phi=\phi(x)$, we define its scaling $\phi_{\mu}$ by

$$
\phi_{\mu}(x):=\mu^{-1} \phi\left(\mu^{-1} x\right),
$$

while for a function $f=f(t, x)$, we define its scaling $f_{\mu}$ by

$$
f_{\mu}(t, x):=\mu^{-1} f\left(\mu^{-2} t, \mu^{-1} x\right) .
$$

Then, given $\phi \in H^{s}\left(\mathbb{R}^{d}\right)$, we have

$$
\left\|\phi_{\mu}\right\|_{\dot{H}^{s}\left(\mathbb{R}^{d}\right)}=\mu^{\frac{d-2}{2}-s}\|\phi\|_{\dot{H}^{s}\left(\mathbb{R}^{d}\right)} .
$$

If $s<s_{\text {crit }}=\frac{d-2}{2}$, that is, if $\phi$ is supercritical with respect to the scaling symmetry, then we can make the $H^{s}$-norm of the scaled function $\phi_{\mu}$ small by taking $\mu \ll 1$. The issue is that the Strichartz estimates we employ in proving probabilistic wellposedness are (sub)critical and do not become small even if we take $\mu \ll 1$. It is for this reason that we consider the randomization $\phi^{\omega, \mu}$ on dilated cubes.

Fix $\phi \in H^{s}\left(\mathbb{R}^{d}\right)$ with $s \in\left(s_{d}, s_{\text {crit }}\right)$, where $s_{\text {crit }}=\frac{d-2}{2}$ and $s_{d}$ is as in (1.11). Let $\phi^{\omega, \mu}$ be its randomization on dilated cubes of scale $\mu$ as in (1.17). Instead of considering (1.1) with $u_{0}=\phi^{\omega, \mu}$, we consider the scaled Cauchy problem

$$
\left\{\begin{array}{l}
i \partial_{t} u_{\mu}+\Delta u_{\mu}= \pm\left|u_{\mu}\right|^{2} u_{\mu}, \\
\left.u_{\mu}\right|_{t=0}=u_{0, \mu}=\left(\phi^{\omega, \mu}\right)_{\mu},
\end{array}\right.
$$

where $u_{\mu}$ is as in (1.2) and $\left(\phi^{\omega, \mu}\right)_{\mu}(x):=\mu^{-1} \phi^{\omega, \mu}\left(\mu^{-1} x\right)$ is the scaled randomization. For notational simplicity, we denote $\left(\phi^{\omega, \mu}\right)_{\mu}$ by $\phi_{\mu}^{\omega, \mu}$ in the following. Denoting the linear and nonlinear part of $u_{\mu}$ by $z_{\mu}(t)=z_{\mu}^{\omega}(t):=S(t) \phi_{\mu}^{\omega, \mu}$ and $v_{\mu}(t):=u_{\mu}(t)-S(t) \phi_{\mu}^{\omega, \mu}$ as before, we reduce (9.2) to

$$
\left\{\begin{array}{l}
i \partial_{t} v_{\mu}+\Delta v_{\mu}= \pm\left|v_{\mu}+z_{\mu}\right|^{2}\left(v_{\mu}+z_{\mu}\right), \\
\left.v_{\mu}\right|_{t=0}=0
\end{array}\right.
$$

Note that if $u$ satisfies (1.1) with initial data $u(0)=\phi^{\omega, \mu}$, then $u_{\mu}, z_{\mu}$, and $v_{\mu}$ are indeed the scalings of $u, z:=S(t) \phi^{\omega, \mu}$, and $v:=u-z$, respectively. For $u_{\mu}$ this simply follows from the scaling symmetry of (1.1). For $z_{\mu}$ and $v_{\mu}$, this follows from the following observation:

$$
\mathcal{F}_{x}\left[\left(S(t) \phi^{\omega, \mu}\right)_{\mu}\right](\xi)=\mu^{d-1} e^{-i \frac{t}{\mu^{2}}|\mu \xi|^{2}} \widehat{\phi^{\omega, \mu}}(\mu \xi)=e^{-i t|\xi|^{2}} \widehat{\phi_{\mu}^{\omega, \mu}}(\xi)=\widehat{z_{\mu}}(t, \xi) .
$$

Define $\Gamma_{\mu}$ by

$$
\Gamma_{\mu} v_{\mu}(t)=\mp i \int_{0}^{t} S\left(t-t^{\prime}\right) \mathcal{N}\left(v_{\mu}+z_{\mu}\right)\left(t^{\prime}\right) d t^{\prime}
$$

In the following, we show that there exists $\mu_{0}=\mu_{0}\left(\varepsilon,\|\phi\|_{H^{s}}\right)>0$ such that, for $\mu \in\left(0, \mu_{0}\right)$, the estimates (4.5) and (4.6) in Proposition 4.1 (with $\widetilde{\Gamma}$ replaced by $\Gamma_{\mu}$ ) hold with $R=\eta_{2}$ outside a set of probability $<\varepsilon$, where $\eta_{2}$ is as in (5.2). In view of (1.16), it is easy to see that

$$
\psi(D-n) \phi_{\mu}=\left(\psi^{\mu}(D-\mu n) \phi\right)_{\mu} .
$$


Hence, we have

$$
\phi_{\mu}^{\omega, \mu}=\left(\phi^{\omega, \mu}\right)_{\mu}=\sum_{n \in \mathbb{Z}^{d}} g_{n}(\omega) \psi(D-n) \phi_{\mu} .
$$

Given $\eta_{2}$ as in (5.2) and $\mu>0$, define $\Omega_{1, \mu}$ by

$$
\Omega_{1, \mu}=\left\{\omega \in \Omega:\left\|S(t) \phi_{\mu}^{\omega, \mu}\right\|_{L_{t}^{q} W_{x}^{s, q}\left(\mathbb{R} \times \mathbb{R}^{d}\right)} \leq \eta_{2}, q=4, \frac{6(d+2)}{d+4}, d+2\right\} .
$$

We also define $\Omega_{2, \mu}$ by

$$
\Omega_{2, \mu}=\left\{\omega \in \Omega:\left\|\phi_{\mu}^{\omega, \mu}\right\|_{H^{s}\left(\mathbb{R}^{d}\right)} \leq \eta_{2}\right\} .
$$

Now, let $\Omega_{\mu}=\Omega_{1, \mu} \cap \Omega_{2, \mu}$. Noting that $4, \frac{6(d+2)}{d+4}$, and $d+2$ are larger than the diagonal Strichartz admissible index $\frac{2(d+2)}{d}$, it follows from Lemma 2.3 and Lemma 2.4 with (9.6) and (9.1) that

$$
P\left(\Omega_{\mu}^{c}\right) \leq C \exp \left(-c \frac{\eta_{2}^{2}}{\left\|\phi_{\mu}\right\|_{H^{s}}^{2}}\right) \leq C \exp \left(-c \frac{\eta_{2}^{2}}{\mu^{d-2-2 s}\|\phi\|_{H^{s}}^{2}}\right)
$$

for $\mu \leq 1$. Then, by setting

$$
\mu_{0} \sim\left(\frac{\eta_{2}}{\|\phi\|_{H^{s}}\left(\log \frac{1}{\varepsilon}\right)^{\frac{1}{2}}}\right)^{\frac{\frac{1}{d-2}}{2}-s}
$$

we have

$$
P\left(\Omega_{\mu}^{c}\right)<\varepsilon
$$

for $\mu \in\left(0, \mu_{0}\right)$. Note that $\mu_{0} \rightarrow 0$ as $\varepsilon \rightarrow 0$. Recall that $q=4, \frac{6(d+2)}{d+4}$, and $d+2$ are the only relevant values of the space-time Lebesgue indices controlling the random forcing term in the proof of Proposition 4.1. Hence, the estimates (4.5) and (4.6) in Proposition 4.1 (with $\widetilde{\Gamma}$ replaced by $\Gamma_{\mu}$ ) hold with $R=\eta_{2}$ for each $\omega \in \Omega_{\mu}$. Then, by repeating the proof of Theorem 1.2 in Section 5 , we see that, for each $\omega \in \Omega_{\mu}$, there exists a global solution $u_{\mu}$ to (9.2) with $\left.u_{\mu}\right|_{t=0}=\phi_{\mu}^{\omega, \mu}$ which scatters both forward and backward in time. By undoing the scaling, we obtain a global solution $u$ to (1.1) with $\left.u\right|_{t=0}=\phi^{\omega, \mu}$ for each $\omega \in \Omega_{\mu}$. Moreover, scattering for $u_{\mu}$ implies scattering for $u$. Indeed, as in Theorem 1.2, there exists $v_{+, \mu} \in H^{\frac{d-2}{2}}\left(\mathbb{R}^{d}\right)$ such that

$$
\lim _{t \rightarrow \infty}\left\|u_{\mu}(t)-S(t)\left(\phi_{\mu}^{\omega, \mu}+v_{+, \mu}\right)\right\|_{H^{\frac{d-2}{2}}}=0 .
$$

Then, a computation analogous to (9.4) yields

$$
S(t)\left(\phi_{\mu}^{\omega, \mu}+v_{+, \mu}\right)=\left(S(t)\left(\phi^{\omega, \mu}+v_{+}\right)\right)_{\mu},
$$

where $v_{+}:=\left(v_{+, \mu}\right)_{\mu^{-1}} \in H^{\frac{d-2}{2}}\left(\mathbb{R}^{d}\right)$. Then, by (9.1), it follows that

$$
\lim _{t \rightarrow \infty}\left\|u-S(t)\left(\phi^{\omega, \mu}+v_{+}\right)\right\|_{H^{\frac{d-2}{2}}}=0 .
$$

This proves that $u$ scatters forward in time. Scattering of $u$ as $t \rightarrow-\infty$ can be proved analogously. This completes the proof of Theorem 1.4. 
Appendix A. On the PROPERTIEs OF The $U^{p}$ - AND $X^{s}$-SPACES

In this appendix, we prove some additional properties of the $U^{p}$ - and $X^{s}$-spaces. In the following, all intervals are half open intervals of the form $[a, b)$ and $p$ denotes a number such that $1 \leq p<\infty$.

Lemma A.1. Let $u=\sum_{j=1}^{\infty} \lambda_{j} a_{j}$, where $\left\{\lambda_{j}\right\}_{j=1}^{\infty} \in \ell^{1}(\mathbb{N} ; \mathbb{C})$ and the $a_{j}$ 's are $U^{p}{ }_{-}$ atoms. Given an interval $I \subset \mathbb{R}$, we can write $u \cdot \chi_{I}$ as $u \cdot \chi_{I}=\sum_{j=1}^{\infty} \widetilde{\lambda}_{j} \widetilde{a}_{j}$ for some $\left\{\widetilde{\lambda}_{j}\right\}_{j=1}^{\infty} \in \ell^{1}$ and some sequence $\left\{\widetilde{a}_{j}\right\}_{j=1}^{\infty}$ of $U^{p}$-atoms such that

$$
\sum_{j=1}^{\infty}\left|\widetilde{\lambda}_{j}\right| \leq \sum_{j=1}^{\infty}\left|\lambda_{j}\right|
$$

As a consequence, we have

$$
\left\|u \cdot \chi_{I}\right\|_{U^{p}(\mathbb{R})} \leq\|u\|_{U^{p}(\mathbb{R})}
$$

for any $u \in U^{p}(\mathbb{R})$ and any $I \subset \mathbb{R}$.

Proof. With $a_{j}=\sum_{k=1}^{K_{j}} \phi_{k-1}^{j} \chi_{\left[t_{k-1}^{j}, t_{k}^{j}\right)}$, we have

$$
u \cdot \chi_{I}=\sum_{j=1}^{\infty} \lambda_{j} a_{j} \chi_{I}=\sum_{j=1}^{\infty} \lambda_{j} \sum_{k=1}^{K_{j}} \phi_{k-1}^{j} \chi_{\left[t_{k-1}^{j}, t_{k}^{j}\right) \cap I} .
$$

Then, setting $\widetilde{\lambda}_{j}$ and $\widetilde{a}_{j}$ as

$$
\begin{aligned}
& \tilde{\lambda}_{j}=\left(\sum_{k \in A_{j}(I)}\left\|\phi_{k}^{j}\right\|_{H}^{p}\right)^{\frac{1}{p}} \lambda_{j}, \\
& \widetilde{a}_{j}=\frac{1}{\left(\sum_{k \in A_{j}(I)}\left\|\phi_{k}^{j}\right\|_{H}^{p}\right)^{\frac{1}{p}}} \sum_{k \in A_{j}(I)} \phi_{k-1}^{j} \chi_{\left[t_{k-1}^{j}, t_{k}^{j}\right) \cap I},
\end{aligned}
$$

where $A_{j}(I)$ is defined by

$$
A_{j}(I)=\left\{k \in\left\{1, \ldots, K_{j}\right\}:\left[t_{k-1}^{j}, t_{k}^{j}\right) \cap I \neq \emptyset\right\},
$$

we have $u \cdot \chi_{I}=\sum_{j=1}^{\infty} \widetilde{\lambda}_{j} \widetilde{a}_{j}$. Moreover, noting that

$$
\left(\sum_{k \in A_{j}(I)}\left\|\phi_{k}^{j}\right\|_{H}^{p}\right)^{\frac{1}{p}} \leq\left(\sum_{k=1}^{K_{j}}\left\|\phi_{k}^{j}\right\|_{H}^{p}\right)^{\frac{1}{p}}=1,
$$

we obtain (A.1) from (A.3). Finally, by (A.1), we have

$$
\left\|u \cdot \chi_{I}\right\|_{U^{p}(\mathbb{R})} \leq \sum_{j=1}^{\infty}\left|\lambda_{j}\right|
$$

for any representation $u=\sum_{j=1}^{\infty} \lambda_{j} a_{j}$ with $\left\{\lambda_{j}\right\}_{j=1}^{\infty} \in \ell^{1}(\mathbb{N} ; \mathbb{C})$ and $U^{p}$-atoms $a_{j}$ 's. Hence, by taking an infimum over all such representations of $u$, we obtain (A.2).

Given an interval $I \subset \mathbb{R}$, we define the local-in-time $U^{p}$-norm in the usual manner as a restriction norm:

$$
\|u\|_{U^{p}(I)}=\inf _{v}\left\{\|v\|_{U^{p}(\mathbb{R})}:\left.v\right|_{I}=u\right\} .
$$

Remark A.2. The infimum is achieved by $v=u \cdot \chi_{I}$ in view of Lemma A.1 In the following, however, we may use other extensions, depending on our purpose. 
The next lemma states the subadditivity of the local-in-time $U^{p}$-norm over intervals.

Lemma A.3. Given an interval $I \subset \mathbb{R}$, let $I=\bigcup_{j=1}^{\infty} I_{j}$ be a partition of $I$. Then, we have

$$
\|u\|_{U^{p}(I)} \leq \sum_{j=1}^{\infty}\|u\|_{U^{p}\left(I_{j}\right)}
$$

Proof. Given $\varepsilon>0$, it follows from the definition of the local-in-time $U^{p}$-norm that there exists $v_{j} \in U^{p}(\mathbb{R})$ such that $\left.v_{j}\right|_{I_{j}}=u$ and

$$
\left\|v_{j}\right\|_{U^{p}(\mathbb{R})} \leq\|u\|_{U^{p}\left(I_{j}\right)}+\frac{\varepsilon}{2^{j}}
$$

for each $j \in \mathbb{N}$. Then, by (A.2) and (A.5), we have

$$
\begin{aligned}
\|u\|_{U^{p}(I)} & \leq\left\|\sum_{j=1}^{\infty} v_{j} \cdot \chi_{I_{j}}\right\|_{U^{p}(\mathbb{R})} \leq \sum_{j=1}^{\infty}\left\|v_{j} \cdot \chi_{I_{j}}\right\|_{U^{p}(\mathbb{R})} \leq \sum_{j=1}^{\infty}\left\|v_{j}\right\|_{U^{p}(\mathbb{R})} \\
& \leq \sum_{j=1}^{\infty}\|u\|_{U^{p}\left(I_{j}\right)}+\varepsilon .
\end{aligned}
$$

Since $\varepsilon>0$ is arbitrary, (A.4) follows from (A.6).

As a corollary, we immediately obtain the following subadditivity property of the local-in-time $X^{s}$-norm over intervals.

Lemma A.4. Let $s \in \mathbb{R}$. Given an interval $I \subset \mathbb{R}$, let $I=\bigcup_{j=1}^{\infty} I_{j}$ be a partition of I. Then, we have

$$
\|u\|_{X^{s}(I)} \leq \sum_{j=1}^{\infty}\|u\|_{X^{s}\left(I_{j}\right)} .
$$

We say that $u$ on $[a, b)$ is a regulated function if both left and right limits exist at every point (including one-sided limits at the endpoint4). Given a regulated function $u$ on $[a, b)$ and a partition $\mathcal{P}=\left\{\tau_{1}, \ldots, \tau_{n}\right\}$ of $[a, b): a<\tau_{1}<\cdots<\tau_{n}<b$, we define a step function $u_{\mathcal{P}}$ by

$$
u_{\mathcal{P}}(t)= \begin{cases}u(t), & \text { if } t=\tau_{j}, \\ u\left(\tau_{j}+\right), & \text { if } \tau_{j}<t<\tau_{j+1},\end{cases}
$$

where we set $\tau_{0}=a$ and $\tau_{n+1}=b$. In particular, if $u$ is right-continuous, we have $u_{\mathcal{P}}(t)=u\left(\tau_{j}\right)$ for $\tau_{j} \leq t<\tau_{j+1}$. Note that the mapping $\mathcal{P}: u \mapsto u_{\mathcal{P}}$ is linear.

Lemma A.5. Let $u \in U^{p}(\mathbb{R})$.

(i) For any partition $\mathcal{P}$ of $\mathbb{R}$, we have

$$
\left\|u_{\mathcal{P}}\right\|_{U^{p}(\mathbb{R})} \leq\|u\|_{U^{p}(\mathbb{R})} .
$$

(ii) Given $\varepsilon>0$, there exists a partition $\mathcal{P}$ of $\mathbb{R}$ such that

$$
\left\|u-u_{\mathcal{P}}\right\|_{U^{p}(\mathbb{R})}<\varepsilon .
$$

\footnotetext{
${ }^{4}$ We allow $a=-\infty$ and/or $b=\infty$.
} 
Proof. (i) We first claim that, given a $U^{p}$-atom $a$, we have $\left\|a_{\mathcal{P}}\right\|_{U^{p}(\mathbb{R})} \leq 1$ for any partition $\mathcal{P}$. Given a $U^{p}$-atom $a=\sum_{k=1}^{K} \phi_{k-1} \chi_{\left[t_{k-1}, t_{k}\right)}$ and a partition $\mathcal{P}=$ $\left\{\tau_{1}, \ldots, \tau_{n}\right\}$ of $\mathbb{R}$, we have

$$
a_{\mathcal{P}}=\sum_{j=1}^{n} a\left(\tau_{j}\right) \chi_{\left[\tau_{j}, \tau_{j+1}\right)}
$$

where $\tau_{n+1}=\infty$. Note that we have

$$
a\left(\tau_{j}\right)= \begin{cases}\phi_{k-1}, & \text { if } \tau_{j} \in\left[t_{k-1}, t_{k}\right) \text { for some } k, \\ 0, & \text { otherwise. }\end{cases}
$$

We can simplify the expression in (A.9) by concatenating neighboring intervals $\left[\tau_{j}, \tau_{j+1}\right)$ and $\left[\tau_{j+1}, \tau_{j+2}\right)$ if $a\left(\tau_{j}\right)=a\left(\tau_{j+1}\right)$ and obtain

$$
a_{\mathcal{P}}=\sum_{\ell=1}^{L} a\left(\tau_{j_{\ell}}\right) \chi_{\left[\tau_{j_{\ell}}, \tau_{j_{\ell+1}}\right)}
$$

for some subpartition $\left\{\tau_{j_{\ell}}\right\}_{\ell=1}^{L}$ of $\mathcal{P}$, where $a\left(\tau_{j_{\ell}}\right)=\phi_{k-1}$ for some $k$ or $a\left(\tau_{j_{\ell}}\right)=0$. Note that, given $k \in\{1, \ldots, K\}$, there exists at most one $\ell \in\{1, \ldots, L\}$ such that $a\left(\tau_{j_{\ell}}\right)=\phi_{k-1}$ (unless $\phi_{k-1}=\phi_{k^{\prime}-1}$ for some $\left.k^{\prime} \neq k\right)$. In particular, we have

$$
\lambda:=\left(\sum_{\ell=1}^{L}\left\|a\left(\tau_{j_{\ell}}\right)\right\|_{H}^{p}\right)^{\frac{1}{p}} \leq\left(\sum_{k=1}^{K}\left\|\phi_{k-1}\right\|_{H}^{p}\right)^{\frac{1}{p}}=1 .
$$

If $\lambda=0$, then $a_{\mathcal{P}}=0$. Otherwise, we have $a_{\mathcal{P}}=\lambda b$, where $b$ is a $U^{p}$-atom given by

$$
b=\sum_{\ell=1}^{L} \frac{a\left(\tau_{j_{\ell}}\right)}{\lambda} \chi_{\left[\tau_{j_{\ell}}, \tau_{j_{\ell+1}}\right)} .
$$

Hence, $\left\|a_{\mathcal{P}}\right\|_{U^{p}(\mathbb{R})} \leq 1$.

Given $u \in U^{p}(\mathbb{R})$, write $u=\sum_{j=1}^{\infty} \lambda_{j} a_{j}$ for some $\left\{\lambda_{j}\right\}_{j=1}^{\infty} \in \ell^{1}$ and some sequence of $\left\{a_{j}\right\}_{j=1}^{\infty}$ of $U^{p}$-atoms. Then, we have

$$
\left\|u_{\mathcal{P}}\right\|_{U^{p}(\mathbb{R})}=\left\|\sum_{j=1}^{\infty} \lambda_{j} \cdot\left(a_{j}\right)_{\mathcal{P}}\right\|_{U^{p}(\mathbb{R})} \leq \sum_{j=1}^{\infty}\left|\lambda_{j}\right|\left\|\left(a_{j}\right)_{\mathcal{P}}\right\|_{U^{p}(\mathbb{R})} \leq \sum_{j=1}^{\infty}\left|\lambda_{j}\right| .
$$

Therefore, we obtain (A.7), since A.10 holds for any $\left\{\lambda_{j}\right\}_{j=1}^{\infty} \in \ell^{1}$ and any sequence of $\left\{a_{j}\right\}_{j=1}^{\infty}$ of $U^{p}$-atoms such that $u=\sum_{j=1}^{\infty} \lambda_{j} a_{j}$.

(ii) Fix a representation $u=\sum_{j=1}^{\infty} \lambda_{j} a_{j}$ for some $\left\{\lambda_{j}\right\}_{j=1}^{\infty} \in \ell^{1}$ and some sequence $\left\{a_{j}\right\}_{j=1}^{\infty}$ of $U^{p}$-atoms. Then, by setting $u_{J}=\sum_{j=1}^{J} \lambda_{j} a_{j}$ for sufficiently large $J$, we have

$$
\left\|u-u_{J}\right\|_{U^{p}(\mathbb{R})} \leq \sum_{j=J+1}^{\infty}\left|\lambda_{j}\right|<\frac{\varepsilon}{2} .
$$

Note that $u_{J}$ is a step function with finitely many jump discontinuities. Now, we define a partition $\mathcal{P}$ by setting $\mathcal{P}=\left\{t \in \mathbb{R}: u_{J}\right.$ is discontinuous at $\left.t\right\}$. Then, by right-continuity of $u_{J}$, we have $u_{J}-\left(u_{J}\right)_{\mathcal{P}}=0$. Hence, from (A.11) and part (i), 
we obtain

$$
\begin{aligned}
\left\|u-u_{\mathcal{P}}\right\|_{U^{p}(\mathbb{R})} & \leq\left\|u-u_{J}\right\|_{U^{p}(\mathbb{R})}+\left\|u_{J}-\left(u_{J}\right)_{\mathcal{P}}\right\|_{U^{p}(\mathbb{R})}+\left\|\left(u-u_{J}\right)_{\mathcal{P}}\right\|_{U^{p}(\mathbb{R})} \\
& <\frac{\varepsilon}{2}+0+\frac{\varepsilon}{2}=\varepsilon .
\end{aligned}
$$

Note that any refinement $\mathcal{P}^{\prime}$ of the partition $\mathcal{P}$ also yields (A.8).

Lemma A.6. Let $I=[a, b) \subset \mathbb{R}$ be an interval. Given $u \in U^{p}(I) \cap C(I ; H)$, the mapping $t \in I \mapsto\|u\|_{U^{p}([a, t))}$ is continuous.

Remark A.7. It follows from the proof that we need the (left-)continuity of $u$ only in proving left-continuity of the mapping $t \in I \mapsto\|u\|_{U^{p}([a, t))}$.

Proof.

- Part 1: Left-continuity. Suppose that the mapping $t \mapsto\|u\|_{U^{p}([a, t))}$ is not leftcontinuous at $t=t_{*} \in(a, b)$. Then, there exist $\varepsilon>0$ and a sequence $\delta_{n} \in\left(0, t_{*}-a\right)$, $\delta_{n} \rightarrow 0$ as $n \rightarrow \infty$, such that

$$
\|u\|_{U^{p}\left(\left[a, t_{*}-\delta_{n}\right)\right)}<\|u\|_{U^{p}\left(\left[a, t_{*}\right)\right)}-\varepsilon .
$$

By definition, for any $\delta \in\left[0, t_{*}-a\right)$, there exists $v_{\delta} \in U^{p}(\mathbb{R})$ such that $\left.v_{\delta}\right|_{\left[a, t_{*}-\delta\right)}$ $=u$ and

$$
\left\|v_{\delta}\right\|_{U^{p}(\mathbb{R})} \leq\|u\|_{U^{p}\left(\left[a, t_{*}-\delta\right)\right)}+\frac{\varepsilon}{4} .
$$

Moreover, in view of Lemma A.1 we can assume that $v_{\delta}=v_{\delta} \cdot \chi_{\left[a, t_{*}-\delta\right)}$. In particular, we have

$$
v_{\delta}=\sum_{j=1}^{\infty} \lambda_{j}^{\delta} a_{j}^{\delta}=\sum_{j=1}^{\infty} \lambda_{j}^{\delta} \sum_{k=1}^{K_{j}^{\delta}} \phi_{k-1}^{\delta, j} \chi_{\left[t_{k-1}^{\delta, j}, t_{k}^{\delta, j}\right)},
$$

where $t_{K_{j}^{\delta}}^{\delta, j} \leq t_{*}-\delta$. Now, we define an extension $\widetilde{v}_{\delta}$ of $v_{\delta}$ onto $\mathbb{R}$ by setting $t_{K_{j}^{\delta}}^{\delta, j}=\infty$ in (A.14) if $t_{K_{j}^{\delta}}^{\delta, j}=t_{*}-\delta$. By continuity of $u$ and $\left.v_{\delta}\right|_{\left[a, t_{*}-\delta\right)}=u$, we have $\widetilde{v}_{\delta}(t)=u\left(t_{*}-\delta\right)$ for $t \in\left[t_{*}-\delta, \infty\right)$. By construction, we have

$$
\left\|v_{\delta}\right\|_{U^{p}(\mathbb{R})}=\left\|\widetilde{v}_{\delta}\right\|_{U^{p}(\mathbb{R})} .
$$

Let $\widetilde{v}$ be the extension of $u \cdot \chi_{\left[a, t_{*}\right)}$ constructed as above with $\delta=0$. Then, by definition of the $U^{p}$-norm and Lemma A.5 (ii), there exists a partition $\mathcal{P}$ of $\mathbb{R}$ such that

$$
\|u\|_{U^{p}\left(\left[a, t_{*}\right)\right)} \leq\|\widetilde{v}\|_{U^{p}(\mathbb{R})} \leq\left\|\widetilde{v}_{\mathcal{P}}\right\|_{U^{p}(\mathbb{R})}+\frac{\varepsilon}{8} .
$$

Since A.16) holds for any refinement $\mathcal{P}^{\prime}$ of $\mathcal{P}$, we can assume that $t_{*} \in \mathcal{P}$.

By uniform continuity of $u$, there exists $\delta_{0}>0$ such that

$$
\left\|u\left(t_{1}\right)-u\left(t_{2}\right)\right\|_{H}<\frac{\varepsilon}{8 \cdot(\# \mathcal{P}+1)}
$$


for any $t_{1}, t_{2} \in\left(t_{*}-\delta_{0}, t_{*}\right]$. Since $\widetilde{v}_{\delta}=\widetilde{v}$ on $\left(-\infty, t_{*}-\delta\right], \widetilde{v}(t)=u\left(t_{*}\right)$ for $t \geq t_{*}$, and $\widetilde{v}_{\delta}(t)=u\left(t_{*}-\delta\right)$ for $t \geq t_{*}-\delta$, we have

$$
\begin{aligned}
\widetilde{v}_{\mathcal{P}}-\left(\widetilde{v}_{\delta}\right)_{\mathcal{P}} & =\sum_{\substack{\tau_{j} \in \mathcal{P} \\
\tau_{j}>t_{*}-\delta}}\left(u\left(\tau_{j}\right)-u\left(t_{*}-\delta\right)\right) \chi_{\left[\tau_{j}, \tau_{j+1}\right)} \\
& =\sum_{\substack{\tau_{j} \in \mathcal{P} \\
\tau_{j} \in\left(t_{*}-\delta, t_{*}\right)}}\left(u\left(\tau_{j}\right)-u\left(t_{*}-\delta\right)\right) \chi_{\left[\tau_{j}, \tau_{j+1}\right)}+\left(u\left(t_{*}\right)-u\left(t_{*}-\delta\right)\right) \chi_{\left[t_{*}, \infty\right)} .
\end{aligned}
$$

Then, from (A.17), we have

$$
\begin{aligned}
\left\|\widetilde{v}_{\mathcal{P}}-\left(\widetilde{v}_{\delta}\right)_{\mathcal{P}}\right\|_{U^{p}(\mathbb{R})} & \leq \sum_{\substack{\tau_{j} \in \mathcal{P} \\
\tau_{j} \in\left(t_{*}-\delta, t_{*}\right)}}\left\|u\left(\tau_{j}\right)-u\left(t_{*}-\delta\right)\right\|_{H}+\left\|u\left(t_{*}\right)-u\left(t_{*}-\delta\right)\right\|_{H} \\
& <\frac{\varepsilon}{8}
\end{aligned}
$$

for any $\delta \in\left(0, \delta_{0}\right)$.

Finally, from (A.16), A.18), Lemma A.5 (i), A.15, A.13), and (A.12), we have

$$
\begin{aligned}
\|u\|_{U^{p}\left(\left[a, t_{*}\right)\right)} & \leq\left\|\left(\widetilde{v}_{\delta_{n}}\right)_{\mathcal{P}}\right\|_{U^{p}(\mathbb{R})}+\frac{\varepsilon}{4} \leq\left\|\widetilde{v}_{\delta_{n}}\right\|_{U^{p}(\mathbb{R})}+\frac{\varepsilon}{4} \leq\|u\|_{U^{p}\left(\left[a, t_{*}-\delta_{n}\right)\right)}+\frac{\varepsilon}{2} \\
& \leq\|u\|_{U^{p}\left(\left[a, t_{*}\right)\right)}-\frac{\varepsilon}{2}
\end{aligned}
$$

for sufficiently large $n$ such that $\delta_{n}<\delta_{0}$. This is a contradiction. Therefore, the mapping $t \mapsto\|u\|_{U^{p}([a, t))}$ is left-continuous at $t=t_{*}$.

- Part 2: Right-continuity. Fix $t_{*} \in I$ and small $\varepsilon>0$. As in Part 1, let $\widetilde{v}_{0}$ be the extension of $v_{0}=v_{0} \cdot \chi_{\left[a, t_{*}\right)}$ satisfying (A.13). In particular, from (A.13) and (A.15), we have

$$
\left\|\widetilde{v}_{0}\right\|_{U^{p}(\mathbb{R})} \leq\|u\|_{U^{p}\left(\left[a, t_{*}\right)\right)}+\frac{\varepsilon}{4} .
$$

Note that $\widetilde{v}_{0}=0$ on $(-\infty, a)$.

Let $w=\widetilde{u}-\widetilde{v}_{0}$, where $\widetilde{u} \in U^{p}(\mathbb{R})$ is an extension of $u$ from $I$ onto $\mathbb{R}$ such that $\tilde{u}=0$ on $(-\infty, a)$. Since $w \in U^{p}(\mathbb{R})$, we can write $w=\sum_{j=1}^{\infty} \lambda_{j} a_{j}$ for some $\left\{\lambda_{j}\right\}_{j=1}^{\infty} \in \ell^{1}$ and some sequence $\left\{a_{j}\right\}_{j=1}^{\infty}$ of $U^{p}$-atoms. Since $w=\widetilde{u}-\widetilde{v}_{0}=0$ on $\left(-\infty, t_{*}\right]$, we can assume that $\operatorname{supp}\left(a_{j}\right) \subset\left(t_{*}, \infty\right)$ for all $j$. Then, we can choose large $J=J(\varepsilon) \in \mathbb{N}$ such that

$$
\sum_{j=J+1}^{\infty}\left|\lambda_{j}\right|<\frac{\varepsilon}{4}
$$

Noting that $w_{J}:=\sum_{j=1}^{J} \lambda_{j} a_{j}$ is a finite linear combination of characteristic functions, there exists $\delta_{0}>0$ such that $w_{J}$ is constant on $\left[t_{*}, t_{*}+\delta_{0}\right) \subset I$. Define $\lambda_{0}$, $\phi$, and $a_{0}$ by

$$
\lambda_{0}:=\left\|w_{J}\left(t_{*}\right)\right\|_{H}, \quad \phi:=\lambda_{0}^{-1} w_{J}\left(t_{*}\right), \quad \text { and } \quad a_{0}:=\chi_{\left[t_{*}, \infty\right)} \phi .
$$

Then, define $\widetilde{w}$ by $\widetilde{w}(t):=w_{J}\left(t_{*}\right) \chi_{\left[t_{*}, \infty\right)}+\sum_{j=J+1}^{\infty} \lambda_{j} a_{j}=\lambda_{0} a_{0}+\sum_{j=J+1}^{\infty} \lambda_{j} a_{j}$. Note that $\widetilde{w}=0$ on $\left(-\infty, t_{*}\right]$. It follows from (A.20) that

$$
\|\widetilde{w}\|_{U^{p}(\mathbb{R})} \leq\left|\lambda_{0}\right|+\sum_{j=J+1}^{\infty}\left|\lambda_{j}\right|<\frac{\varepsilon}{2},
$$


since we have

$$
\lambda_{0}=\left\|w\left(t_{*}\right)-\sum_{j=J+1}^{\infty} \lambda_{j} a_{j}\left(t_{*}\right)\right\|_{H}=\left\|\sum_{j=J+1}^{\infty} \lambda_{j} a_{j}\left(t_{*}\right)\right\|_{H} \leq \sum_{j=J+1}^{\infty}\left|\lambda_{j}\right|<\frac{\varepsilon}{4} .
$$

Here, we used the fact that $\left\|a_{j}\right\|_{H} \leq 1$ for a $U^{p}$-atom $a$. By construction, we have $\operatorname{supp}(\widetilde{w}) \subset\left[t_{*}, \infty\right)$. Then, noting that $u-\widetilde{v}_{0}=\widetilde{w}$ on $\left(-\infty, t_{*}+\delta_{0}\right) \subset I$, it follows from (A.19) and (A.21) that

$$
\begin{aligned}
\|u\|_{U^{p}\left(\left[a, t_{*}+\delta\right)\right)} & \leq\left\|\widetilde{v}_{0}\right\|_{U^{p}\left(\left[a, t_{*}+\delta\right)\right)}+\|\widetilde{w}\|_{U^{p}\left(\left[a, t_{*}+\delta\right)\right)} \leq\left\|\widetilde{v}_{0}\right\|_{U^{p}(\mathbb{R})}+\|\widetilde{w}\|_{U^{p}(\mathbb{R})} \\
& \leq\|u\|_{U^{p}\left(\left[a, t_{*}\right)\right)}+\frac{3}{4} \varepsilon
\end{aligned}
$$

for any $\delta \in\left(0, \delta_{0}\right]$. Therefore, the mapping $t \mapsto\|u\|_{U^{p}([a, t))}$ is right-continuous at $t=t_{*}$.

Lemma A.8. Let $s \in \mathbb{R}$ and $I=[a, b) \subset \mathbb{R}$. Given $u \in X^{s}(I) \cap C\left(I ; H^{s}\left(\mathbb{R}^{d}\right)\right)$, the mapping $t \in I \mapsto\|u\|_{X^{s}([a, t))}$ is continuous.

Proof. First, we claim that the infimum in the definition of the local-in-time $X^{s}$ norm on an interval $[a, t)$ is achieved by $u \cdot \chi_{[a, t)}$ for any $t \leq b$. Namely, we have

$$
\|u\|_{X^{s}([a, t))}=\left\|u \cdot \chi_{[a, t)}\right\|_{X^{s}(\mathbb{R})} .
$$

On the one hand, given any extension $v$ on $\mathbb{R}$ of $u$ restricted to $[a, t)$, i.e. $\left.v\right|_{[a, t)}=u$, we have

$$
\|u\|_{X^{s}([a, t))} \leq\|v\|_{X^{s}(\mathbb{R})} .
$$

On the other hand, by Lemma A.1, we have

$$
\left\|u \cdot \chi_{[a, t)}\right\|_{X^{s}(\mathbb{R})}=\left\|v \cdot \chi_{[a, t)}\right\|_{X^{s}(\mathbb{R})} \leq\|v\|_{X^{s}(\mathbb{R})} .
$$

Hence, A.22 follows, since (A.23) and A.24 hold for any extension v. Moreover, we have

$$
\begin{aligned}
\|u\|_{X^{s}([a, t))} & =\left\|u \cdot \chi_{[a, t)}\right\|_{X^{s}(\mathbb{R})}=\left(\sum_{\substack{N \geq 1 \\
\text { dyadic }}} N^{2 s}\left\|\mathbf{P}_{N} u \cdot \chi_{[a, t)}\right\|_{U_{\Delta}^{2}\left(\mathbb{R} ; L^{2}\right)}^{2}\right)^{\frac{1}{2}} \\
& =\left(\sum_{\substack{N \geq 1 \\
\text { dyadic }}} N^{2 s}\left\|\mathbf{P}_{N} u\right\|_{U_{\Delta}^{2}\left([a, t) ; L^{2}\right)}^{2}\right)^{\frac{1}{2}}
\end{aligned}
$$

where the last equality follows from Remark A.2

Let $v$ be an extension of $u$ onto $\mathbb{R}$ such that $\|v\|_{X^{s}(\mathbb{R})}<\infty$. Given $\varepsilon>0$, we can choose $J \in \mathbb{N}$ such that

$$
\left(\sum_{j=J}^{\infty} 2^{2 j s}\left\|\mathbf{P}_{2^{j}} v\right\|_{U_{\Delta}^{2}\left(\mathbb{R} ; L^{2}\right)}^{2}\right)^{\frac{1}{2}}<\frac{\varepsilon}{4}
$$

Then, we have

$$
\left(\sum_{j=J}^{\infty} 2^{2 j s}\left\|\mathbf{P}_{2^{j}} u\right\|_{U_{\Delta}^{2}\left([a, t) ; L^{2}\right)}^{2}\right)^{\frac{1}{2}}<\frac{\varepsilon}{4}
$$


for any $t \in I$. Fix $t_{*} \in I$. By Lemma A.6, for each $j=0,1, \ldots, J-1$, there exists $\delta_{j}>0$ such that

$$
2^{j s}\left|\left\|\mathbf{P}_{2^{j}} u\right\|_{U_{\Delta}^{2}\left(\left[a, t_{*}\right) ; L^{2}\right)}-\left\|\mathbf{P}_{2^{j}} u\right\|_{U_{\Delta}^{2}\left(\left[a, t_{*}+\delta\right) ; L^{2}\right)}\right|<\frac{\varepsilon}{2 J^{\frac{1}{2}}}
$$

for $|\delta|<\delta_{j}$. Then, by Minkowski's inequality with (A.25), (A.26), and (A.27), we have

$$
\begin{aligned}
\mid\|u\|_{X^{s}\left(\left[a, t_{*}\right)\right)} & -\|u\|_{X^{s}\left(\left[a, t_{*}+\delta\right)\right)} \mid \\
& \leq\left(\sum_{j=0}^{\infty} 2^{2 j s}\left(\left\|\mathbf{P}_{2^{j}} u\right\|_{U_{\Delta}^{2}\left(\left[a, t_{*}\right) ; L^{2}\right)}-\left\|\mathbf{P}_{2^{j}} u\right\|_{U_{\Delta}^{2}\left(\left[a, t_{*}+\delta\right) ; L^{2}\right)}\right)^{2}\right)^{\frac{1}{2}} \\
& \leq J^{\frac{1}{2}} \max _{j=0, \ldots, J-1} 2^{j s}\left|\left\|\mathbf{P}_{2^{j}} u\right\|_{U_{\Delta}^{2}\left(\left[a, t_{*}\right) ; L^{2}\right)}-\left\|\mathbf{P}_{2^{j}} u\right\|_{U_{\Delta}^{2}\left(\left[a, t_{*}+\delta\right) ; L^{2}\right)}\right|+\frac{\varepsilon}{2} \\
& <\varepsilon
\end{aligned}
$$

for $0<|\delta|<\min \left(\delta_{0}, \ldots, \delta_{J-1}\right)$. This proves the lemma.

\section{REFERENCES}

[1] Thomas Alazard and Rémi Carles, Loss of regularity for supercritical nonlinear Schrödinger equations, Math. Ann. 343 (2009), no. 2, 397-420, DOI 10.1007/s00208-008-0276-6. MR2461259(2009j:35341)

[2] Antoine Ayache and Nikolay Tzvetkov, $L^{p}$ properties for Gaussian random series, Trans. Amer. Math. Soc. 360 (2008), no. 8, 4425-4439, DOI 10.1090/S0002-9947-08-04456-5. MR2395179 (2009e:30006)

[3] Árpád Bényi and Tadahiro Oh, Modulation spaces, Wiener amalgam spaces, and Brownian motions, Adv. Math. 228 (2011), no. 5, 2943-2981, DOI 10.1016/j.aim.2011.07.023. MR2838066 (2012g:60258)

[4] Árpad Bényi, Tadahiro Oh, and Oana Pocovnicu, Wiener randomization on unbounded domains and an application to almost sure well-posedness of NLS, to appear in Excursions in Harmonic Analysis.

[5] Jean Bourgain, Fourier transform restriction phenomena for certain lattice subsets and applications to nonlinear evolution equations. I. Schrödinger equations, Geom. Funct. Anal. 3 (1993), no. 2, 107-156, DOI 10.1007/BF01896020. MR1209299 (95d:35160a)

[6] Jean Bourgain, Invariant measures for the 2D-defocusing nonlinear Schrödinger equation, Comm. Math. Phys. 176 (1996), no. 2, 421-445. MR1374420 (96m:35292)

[7] Jean Bourgain, Invariant measures for the Gross-Piatevskii equation, J. Math. Pures Appl. (9) 76 (1997), no. 8, 649-702, DOI 10.1016/S0021-7824(97)89965-5. MR1470880(98h:58102)

[8] Jean Bourgain, Refinements of Strichartz' inequality and applications to $2 D-N L S$ with critical nonlinearity, Internat. Math. Res. Notices 5 (1998), 253-283, DOI 10.1155/S1073792898000191. MR1616917 (99f:35184)

[9] Jean Bourgain and Aynur Bulut, Almost sure global well-posedness for the radial nonlinear Schrödinger equation on the unit ball II: the 3d case, J. Eur. Math. Soc. (JEMS) 16 (2014), no. 6, 1289-1325, DOI 10.4171/JEMS/461. MR3226743

[10] Jean Bourgain and Aynur Bulut, Invariant Gibbs measure evolution for the radial nonlinear wave equation on the 3d ball, J. Funct. Anal. 266 (2014), no. 4, 2319-2340, DOI 10.1016/j.jfa.2013.06.002. MR.3150162

[11] Nicolas Burq, Patrick Gérard, and Nikolay Tzvetkov, Multilinear eigenfunction estimates and global existence for the three dimensional nonlinear Schrödinger equations (English, with English and French summaries), Ann. Sci. École Norm. Sup. (4) 38 (2005), no. 2, 255-301, DOI 10.1016/j.ansens.2004.11.003. MR2144988 (2006m:35337)

[12] Nicolas Burq, Laurent Thomann, and Nikolay Tzvetkov, Long time dynamics for the one dimensional non linear Schrödinger equation (English, with English and French summaries), Ann. Inst. Fourier (Grenoble) 63 (2013), no. 6, 2137-2198. MR3237443

[13] Nicolas Burq, Laurent Thomann, and Nikolay Tzvetkov, Global infinite energy solutions for the cubic wave equation, to appear in Bull. Soc. Math. France. 
[14] Nicolas Burq and Nikolay Tzvetkov, Random data Cauchy theory for supercritical wave equations. I. Local theory, Invent. Math. 173 (2008), no. 3, 449-475, DOI 10.1007/s00222-0080124-z. MR2425133 (2009k:58057)

[15] Nicolas Burq and Nikolay Tzvetkov, Probabilistic well-posedness for the cubic wave equation, J. Eur. Math. Soc. (JEMS) 16 (2014), no. 1, 1-30, DOI 10.4171/JEMS/426. MR3141727

[16] Rémi Carles, Geometric optics and instability for semi-classical Schrödinger equations, Arch. Ration. Mech. Anal. 183 (2007), no. 3, 525-553, DOI 10.1007/s00205-006-0017-5. MR2278414 (2007j:35211)

[17] Thierry Cazenave and Fred B. Weissler, Some remarks on the nonlinear Schrödinger equation in the critical case, Nonlinear semigroups, partial differential equations and attractors (Washington, DC, 1987), Lecture Notes in Math., vol. 1394, Springer, Berlin, 1989, pp. 18-29, DOI 10.1007/BFb0086749. MR.1021011 (91a:35149)

[18] Michael Christ, James Colliander, and Terrence Tao, Asymptotics, frequency modulation, and low regularity ill-posedness for canonical defocusing equations, Amer. J. Math. 125 (2003), no. 6, 1235-1293. MR2018661 (2005d:35223)

[19] James Colliander, M. Keel, G. Staffilani, H. Takaoka, and T. Tao, Global well-posedness and scattering for the energy-critical nonlinear Schrödinger equation in $\mathbb{R}^{3}$, Ann. of Math. (2) 167 (2008), no. 3, 767-865, DOI 10.4007/annals.2008.167.767. MR2415387 (2009f:35315)

[20] James Colliander and Tadahiro Oh, Almost sure well-posedness of the cubic nonlinear Schrödinger equation below $L^{2}(\mathbb{T})$, Duke Math. J. 161 (2012), no. 3, 367-414, DOI 10.1215/00127094-1507400. MR2881226

[21] Yu Deng, Two-dimensional nonlinear Schrödinger equation with random radial data, Anal. PDE 5 (2012), no. 5, 913-960. MR 3022846

[22] Anne-Sophie de Suzzoni, Invariant measure for the cubic wave equation on the unit ball of $\mathbb{R}^{3}$, Dyn. Partial Differ. Equ. 8 (2011), no. 2, 127-147, DOI 10.4310/DPDE.2011.v8.n2.a4. MR2857361 (2012k:35345)

[23] Anne-Sophie de Suzzoni, Consequences of the choice of a particular basis of $L^{2}\left(S^{3}\right)$ for the cubic wave equation on the sphere and the Euclidean space, Commun. Pure Appl. Anal. 13 (2014), no. 3, 991-1015, DOI 10.3934/cpaa.2014.13.991. MR.3177685

[24] Hans Feichtinger, Modulation spaces of locally compact Abelian groups, Technical report, University of Vienna (1983). in Proc. Internat. Conf. on Wavelets and Applications (Chennai, 2002), R. Radha, M. Krishna, S. Thangavelu (eds.), New Delhi Allied Publishers (2003), 1-56.

[25] Hans G. Feichtinger and K. H. Gröchenig, Banach spaces related to integrable group representations and their atomic decompositions. I, J. Funct. Anal. 86 (1989), no. 2, 307-340, DOI 10.1016/0022-1236(89)90055-4. MR1021139 (91g:43011)

[26] Hans G. Feichtinger and K. H. Gröchenig, Banach spaces related to integrable group representations and their atomic decompositions. II, Monatsh. Math. 108 (1989), no. 2-3, 129-148, DOI 10.1007/BF01308667. MR:1026614 (91g:43012)

[27] J. Ginibre and G. Velo, Smoothing properties and retarded estimates for some dispersive evolution equations, Comm. Math. Phys. 144 (1992), no. 1, 163-188. MR.1151250 (93a:35065)

[28] Karlheinz Gröchenig, Foundations of time-frequency analysis, Applied and Numerical Harmonic Analysis, Birkhäuser Boston, Inc., Boston, MA, 2001. MR1843717 (2002h:42001)

[29] Martin Hadac, Sebastian Herr, and Herbert Koch, Well-posedness and scattering for the KPII equation in a critical space, Ann. Inst. H. Poincaré Anal. Non Linéaire 26 (2009), no. 3, 917-941, DOI 10.1016/j.anihpc.2008.04.002. MR2526409(2010d:35301)

[30] Sebastian Herr, Daniel Tataru, and Nikolay Tzvetkov, Global well-posedness of the energycritical nonlinear Schrödinger equation with small initial data in $H^{1}\left(\mathbb{T}^{3}\right)$, Duke Math. J. 159 (2011), no. 2, 329-349, DOI 10.1215/00127094-1415889. MR2824485(2012j:35392)

[31] Alexandru D. Ionescu and Benoit Pausader, Global well-posedness of the energy-critical defocusing NLS on $\mathbb{R} \times \mathbb{T}^{3}$, Comm. Math. Phys. 312 (2012), no. 3, 781-831, DOI 10.1007/s00220012-1474-3. MR2925134

[32] Jean-Pierre Kahane, Some random series of functions, 2nd ed., Cambridge Studies in Advanced Mathematics, vol. 5, Cambridge University Press, Cambridge, 1985. MR833073 (87m:60119)

[33] Markus Keel and Terence Tao, Endpoint Strichartz estimates, Amer. J. Math. 120 (1998), no. 5, 955-980. MR1646048(2000d:35018) 
[34] Carlos E. Kenig and Frank Merle, Global well-posedness, scattering and blow-up for the energy-critical, focusing, non-linear Schrödinger equation in the radial case, Invent. Math. 166 (2006), no. 3, 645-675, DOI 10.1007/s00222-006-0011-4. MR2257393 (2007g:35232)

[35] Carlos E. Kenig and Frank Merle, Scattering for $\dot{H}^{1 / 2}$ bounded solutions to the cubic, defocusing NLS in 3 dimensions, Trans. Amer. Math. Soc. 362 (2010), no. 4, 1937-1962, DOI 10.1090/S0002-9947-09-04722-9. MR.2574882 (2011b:35486)

[36] Carlos E. Kenig and Frank Merle, Nondispersive radial solutions to energy supercritical nonlinear wave equations, with applications, Amer. J. Math. 133 (2011), no. 4, 1029-1065, DOI 10.1353/ajm.2011.0029. MR2823870 (2012i:35244)

[37] Rowan Killip, Tadahiro Oh, Oana Pocovnicu, and Monica Vişan, Global well-posedness of the Gross-Pitaevskii and cubic-quintic nonlinear Schrödinger equations with nonvanishing boundary conditions, Math. Res. Lett. 19 (2012), no. 5, 969-986, DOI 10.4310/MRL.2012.v19.n5.a1. MR3039823

[38] Rowan Killip and Monica Vişan, Nonlinear Schrödinger equations at critical regularity, Evolution equations, Clay Math. Proc., vol. 17, Amer. Math. Soc., Providence, RI, 2013, pp. 325437. MR3098643

[39] Rowan Killip and Monica Vişan, Energy-supercritical NLS: critical $\dot{H}^{s}$-bounds imply scattering, Comm. Partial Differential Equations 35 (2010), no. 6, 945-987, DOI 10.1080/03605301003717084. MR2753625 (2011m:35357)

[40] Masaharu Kobayashi and Mitsuru Sugimoto, The inclusion relation between Sobolev and modulation spaces, J. Funct. Anal. 260 (2011), no. 11, 3189-3208, DOI 10.1016/j.jfa.2011.02.015. MR2776566 (2012d:46084)

[41] Herbert Koch and Daniel Tataru, A priori bounds for the $1 D$ cubic NLS in negative Sobolev spaces, Int. Math. Res. Not. IMRN 16 (2007), Art. ID rnm053, 36, DOI 10.1093/imrn/rnm053. MR2353092(2010d:35307)

[42] Jonas Lührmann and Dana Mendelson, Random data Cauchy theory for nonlinear wave equations of power-type on $\mathbb{R}^{3}$, Comm. Partial Differential Equations 39 (2014), no. 12, 2262-2283, DOI 10.1080/03605302.2014.933239. MR3259556

[43] Andrea R. Nahmod, Nataša Pavlović, and Gigliola Staffilani, Almost sure existence of global weak solutions for supercritical Navier-Stokes equations, SIAM J. Math. Anal. 45 (2013), no. 6, 3431-3452, DOI 10.1137/120882184. MR 3131480

[44] Andrea R. Nahmod and Gigliola Staffilani, Almost sure well-posedness for the periodic 3D quintic nonlinear Schrödinger equation below the energy space, to appear in J. Eur. Math. Soc.

[45] Tadahiro Oh, Remarks on nonlinear smoothing under randomization for the periodic $K d V$ and the cubic Szegö equation, Funkcial. Ekvac. 54 (2011), no. 3, 335-365, DOI 10.1619/fesi.54.335. MR.2918143

[46] Tadahiro Oh and Oana Pocovnicu, Probabilistic global well-posedness of the energy-critical defocusing quintic nonlinear wave equation on $\mathbb{R}^{3}$, arXiv:1502.00575 [math.AP].

[47] Kasso A. Okoudjou, Embedding of some classical Banach spaces into modulation spaces, Proc. Amer. Math. Soc. 132 (2004), no. 6, 1639-1647 (electronic), DOI 10.1090/S0002-993904-07401-5. MR2051124(2005b:46074)

[48] T. Ozawa and Y. Tsutsumi, Space-time estimates for null gauge forms and nonlinear Schrödinger equations, Differential Integral Equations 11 (1998), no. 2, 201-222. MR.1741843 (2000m:35167)

[49] R.E.A.C. Paley and A. Zygmund, On some series of functions (1), (2), (3), Proc. Cambridge Philos. Soc. 26 (1930), 337-357, 458-474; 28 (1932), 190-205.

[50] Oana Pocovnicu, Probabilistic global well-posedness of the energy-critical defocusing cubic nonlinear wave equations on $\mathbb{R}^{4}$, to appear in J. Eur. Math. Soc. (JEMS).

[51] Aurélien Poiret, Didier Robert, and Laurent Thomann, Probabilistic global well-posedness for the supercritical nonlinear harmonic oscillator, Anal. PDE 7 (2014), no. 4, 997-1026, DOI 10.2140/apde.2014.7.997. MR3254351

[52] G. Richards, Invariance of the Gibbs measure for the periodic quartic $g K d V$, to appear in Ann. Inst. H. Poincaré Anal. Non Linéaire.

[53] E. Ryckman and M. Vişan, Global well-posedness and scattering for the defocusing energycritical nonlinear Schrödinger equation in $\mathbb{R}^{1+4}$, Amer. J. Math. 129 (2007), no. 1, 1-60, DOI 10.1353/ajm.2007.0004. MR2288737 (2007k:35474) 
[54] Robert S. Strichartz, Restrictions of Fourier transforms to quadratic surfaces and decay of solutions of wave equations, Duke Math. J. 44 (1977), no. 3, 705-714. MR0512086 (58 \#23577)

[55] Mitsuru Sugimoto and Naohito Tomita, The dilation property of modulation spaces and their inclusion relation with Besov spaces, J. Funct. Anal. 248 (2007), no. 1, 79-106, DOI 10.1016/j.jfa.2007.03.015. MR2329683 (2009a:46064)

[56] Terence Tao and Monica Vişan, Stability of energy-critical nonlinear Schrödinger equations in high dimensions, Electron. J. Differential Equations (2005), No. 118, 28. MR2174550 (2006e:35307)

[57] Terence Tao, Monica Vişan, and Xiaoyi Zhang, The nonlinear Schrödinger equation with combined power-type nonlinearities, Comm. Partial Differential Equations 32 (2007), no. 79, 1281-1343, DOI 10.1080/03605300701588805. MR2354495(2009f:35324)

[58] Laurent Thomann, Random data Cauchy problem for supercritical Schrödinger equations (English, with English and French summaries), Ann. Inst. H. Poincaré Anal. Non Linéaire 26 (2009), no. 6, 2385-2402, DOI 10.1016/j.anihpc.2009.06.001. MR2569900 (2010m:35494)

[59] Joachim Toft, Convolutions and embeddings for weighted modulation spaces, Advances in pseudo-differential operators, Oper. Theory Adv. Appl., vol. 155, Birkhäuser, Basel, 2004, pp. 165-186. MR2090373(2005e:42072)

[60] Monica Vişan, Global well-posedness and scattering for the defocusing cubic nonlinear Schrödinger equation in four dimensions, Int. Math. Res. Not. IMRN 5 (2012), 1037-1067. MR2899959

[61] Norbert Wiener, Tauberian theorems, Ann. of Math. (2) 33 (1932), no. 1, 1-100, DOI 10.2307/1968102. MR 1503035

[62] Kenji Yajima, Existence of solutions for Schrödinger evolution equations, Comm. Math. Phys. 110 (1987), no. 3, 415-426. MR891945 (88e:35048)

[63] Ting Zhang and Daoyuan Fang, Random data Cauchy theory for the generalized incompressible Navier-Stokes equations, J. Math. Fluid Mech. 14 (2012), no. 2, 311-324, DOI 10.1007/s00021-011-0069-7. MR2925111

Department of Mathematics, Western Washington University, 516 High Street, Bellingham, WASHington 98225

E-mail address: arpad.benyi@wwu.edu

School of Mathematics, The University of Edinburgh - And - The Maxwell Institute for the Mathematical Sciences, James Clerk Maxwell Bullding, The King's Buildings, Peter Guthrie Tait Road, Edinburgh, EH9 3FD, United Kingdom

E-mail address: hiro.oh@ed.ac.uk

School of Mathematics, Institute for Advanced Study, Einstein Drive, Princeton, New Jersey 08540 - And - Department of Mathematics, Fine Hall, Princeton University, Washington Road, Princeton, New Jersey 08544

E-mail address: opocovnicu@math.princeton.edu 